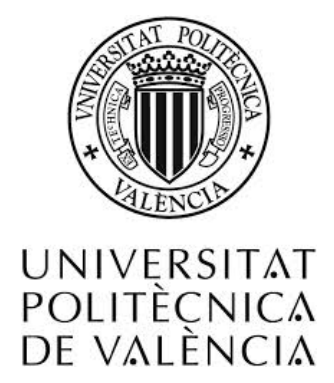

\title{
Soft Set Theory: Generalizations, \\ Fixed Point Theorems, and Applications
}

\author{
Memoria presentada por \\ MuJAhid ABBas \\ para optar al Grado de Doctor por la \\ Universitat Politècnica de VALÈnCIA \\ Dirigida por el Doctor \\ D. Salvador Romaguera Bonilla
}

Valencia, Octubre 2014 

D. Salvador Romaguera Bonilla, Catedrático del Departamento de Matemática Aplicada de la Universitat Politècnica de València

CERTIFICO: que la presente memoria "Soft Set Theory: Generalizations, Fixed Point Theorems, and Applications" ha sido realizada bajo mi dirección por D. Mujahid Abbas, en el Departamento de Matemática Aplicada de la Universitat Politècnica de València, y constituye su tesis para optar al grado de Doctor por la Universitat Politècnica de València.

Y para que así conste, presento la referida tesis, firmando el presente certificado.

Valencia, Octubre de 2014

Fdo. Salvador Romaguera Bonilla 


\section{Agradecimientos}

All thanks are due to "Omniscient" Who inculcated in a man insatiable desire to fathom the mysteries of limitless universe of knowledge.

I greatly admire and am deeply indebted to, Professor Salvador Romaguera, my supervisor, for his valuable comments, unfailing support, suggestions and discussions that he made, from time to time, on and about my work.

I shall be failing my duty if I don't express my heart felt thanks to my reverend teachers and mentors who had always been the source of inspiration for me, and my students I have learned much from them.

My deepest gratitude and thanks are also due to my family members who always encouraged me and refreshed my energies with their sweet words. I especially appreciate my wife and children for their selfless support, full cooperation, understanding and forbearance while I was busy in accomplishment of this project. My sincerest thanks are for my friends, who extended their moral support when I was in dire need of it.

Finally, I gratefully acknowledge Departamento de Matematica Pura y Aplica, Universitat Politecnica de Valencia, Valencia Spain. This great seat of learning enabled me to carry out my research work smoothly. 


\section{Dedicated to, My wife Shagufta \& Children Aon and Rafay}




\title{
RESUMEN ESPAÑOL
}

\author{
Mujahid Abbas
}

Numerosos problemas de la ingeniería, ciencia de la computación, economía, medicina o ciencias sociales se abordan con garantías recurriendo a la construcción de modelos matemáticos adecuados. Sin embargo, los métodos y herramientas que proporciona la matemática clásica no son válidos para atacar diversos problemas del mundo real en los que surgen dificultades derivadas de la aparición de elementos de incerteza e imprecisión en los datos proporcionados. En tales casos, es útil recurrir a nuevas teorías matemáticas con la teoría de de la probabilidad o la de conjuntos difusos. No obstante, estas teorías adolecen de ciertas deficiencias derivadas de cuestiones relativas a la parametrización. La teoría de conjuntos imprecisos ("Soft set theory") proporciona suficientes herramientas en forma de parámetros para tratar la incerteza de los datos de un modo conveniente. Así, la ventaja que presenta la teoría de conjuntos imprecisos respecto de la teoría de probabilidad y la teoría de conjuntos difusos, es que no se maneja cantidades exactas lo que facilita las aplicaciones a la teoría de la decisión, análisis de la demanda, ciencias de la información, matemáticas, y otras disciplinas.

En esta tesis estudiaremos diversas propiedades algebraicas y topológicas de los conjuntos imprecisos y de los conjuntos imprecisos difusos. Como los conjuntos difusos se pueden considerar como funciones multivaluadas, también investigaremos la teoría del punto fijo para funciones en espacios topológicos imprecisos y otras estructuras relacionadas.

Las contribuciones que aportamos en esta tesis a dicho estudio, se resumen a continuación:

i) Revisión de las operaciones básicas en la teoría de conjuntos soft. En particular, demostramos resultados nuevos a partir de las modifica- 
ciones propuestas, que proporcionan una dimensión nueva para explorar esta teoría en el futuro siguiendo distintas direcciones. Nuestro enfoque se puede aplicar al desarrollo y modificació n de la literatura existente sobre espacios topológicos soft.

ii) Definición de nuevas clases de funciones y demostración de la existencia y unicidad, en su caso, de punto fijo para las mismas. De esta forma, presentamos diversos avances en la teoría de punto fijo para espacios métricos.

iii) Inicio de una teoría de punto fijo soft en el contexto de los espacios métricos soft, obteniendo resultados que permiten enlazar la teoría de conjuntos soft con la teoría de punto fijo.

iv) Este estudio tambie constituye un punto de partida para posteriores investigaciones en la teoría "fuzzy soft" de punto fijo. 


\title{
RESUMEN VALENCIANO
}

\author{
Mujahid Abbas
}

Nombrosos problemes de l'enginyeria, ciència de la computació, economia, medicina o ciències socials s'aborden amb garanties recorrent a la construcció de models matemàtics adients. Tanmateix, els mètodes i eines que proporciona la matemàtica clàssica no són vàlids per a atacar diversos problemes del món real en els que sorgixen dificultats derivades de l'aparició d'elements d'incertesa i imprecisió en les dades proporcionades.

En tals casos, és útil recórrer a noves teories matemàtiques com son la teoria de la probabilitat o la de conjunts difusos. No obstant això, estes teories patixen certes deficiències derivades de qüestions relatives a la parametrització. La teoria de conjunts imprecisos ("Soft set theory") proporciona suficients eines en forma de paràmetres per a tractar la incertesa de les dades d'una manera convenient. Així, l'avantage que presenta la teoria de conjunts imprecisos respecte de la teoria de la probabilitat i la teoria de conjunts difusos, és que no maneja quantitats exactes, la qual cosa facilita les aplicacions a la teoria de la decisió, anàlisi de la demanda, ciències de la informació, matemàtiques i atres disciplines.

En esta tesi estudiarem diverses propietats algebraiques i topològiques dels conjunts imprecisos i dels conjunts imprecisos difusos.

Com que els conjunts difusos es poden considerar com a funcions multivaluades, també investigarem la teoria del punt fix per a funcions en espais topològics imprecisos i atres estructures relacionades.

Les contribucions que aportem en esta tesi a tal estudi es resumixen a continuació:

i) Revisió de les operacions bàsiques en la teoria de conjunts soft. En particular, demostrem resultats nous a partir de les modificacions proposades, que proporcionen una dimensió nova per a explorar esta teoria 
en el futur seguint distintes direccions. El nostre enfocament es pot aplicar al desenrollament i modificació de la literatura existent sobre espais topològics soft.

ii) Definició de noves classes de funcions i demostració de l'existència i unicitat, si escau, de punt fix per a d'elles. D'esta forma, presentem diversos avanços en la teoria de punt fix per a espais mètrics.

iii) Inici d'una teoria de punt fix soft en el context dels espais mètrics soft, obtenint resultats que permeten enllaçar la teoria de conjunts soft amb la teoria de punt fix.

iv) Este estudi també constituix un punt de partida per a posteriors investigacions en la teoria "fuzzy soft" de punt fix. 


\title{
RESUMEN INGLES
}

\author{
Mujahid Abbas
}

Mathematical models have extensively been used in problems related to engineering, computer sciences, economics, social, natural and medical sciences etc. It has become very common to use mathematical tools to solve, study the behavior and different aspects of a system and its different subsystems. Because of various uncertainties arising in real world situations, methods of classical mathematics may not be successfully applied to solve them. Thus, new mathematical theories such as probability theory and fuzzy set theory have been introduced by mathematicians and computer scientists to handle the problems associated with the uncertainties of a model. But there are certain deficiencies pertaining to the parametrization in fuzzy set theory. Soft set theory aims to provide enough tools in the form of parameters to deal with the uncertainty in a data and to represent it in a useful way. The distinguishing attribute of soft set theory is that unlike probability theory and fuzzy set theory, it does not uphold a precise quantity. This attribute has facilitated applications in decision making, demand analysis, forecasting, information sciences, mathematics and other disciplines.

In this thesis we will discuss several algebraic and topological properties of soft sets and fuzzy soft sets. Since soft sets can be considered as setvalued maps, the study of fixed point theory for multivalued maps on soft topological spaces and on other related structures will be also explored.

The contributions of the study carried out in this thesis can be summarized as follows:

i) Revisit of basic operations in soft set theory and proving some new results based on these modifications which would certainly set a new dimension to explore this theory further and would help to extend its limits further in different directions. Our findings can be applied to develop and modify the existing literature on soft topological spaces 
ii) Defining some new classes of mappings and then proving the existence and uniqueness of such mappings which can be viewed as a positive contribution towards an advancement of metric fixed point theory

iii) Initiative of soft fixed point theory in framework of soft metric spaces and proving the results lying at the intersection of soft set theory and fixed point theory which would help in establishing a bridge between these two flourishing areas of research.

iv) This study is also a starting point for the future research in the area of fuzzy soft fixed point theory. 


\section{Contents}

1 Introduction. Objectives $\quad 3$

1.1 Background of study . . . . . . . . . . . . . . 3

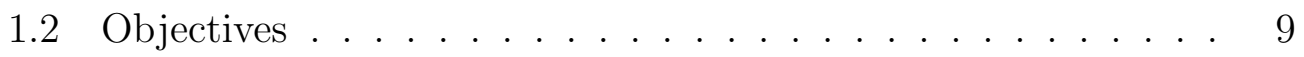

2 On Generalized Soft Equality and Soft Lattice Structure 13

2.1 Preliminaries . . . . . . . . . . . . . . 14

2.2 Generalized soft equality (or $g$-soft equality) relation $\approx_{g} \ldots 18$

2.3 Lattice structure on the soft sets . . . . . . . . . . . . . . . . 21

3 Fixed Points of Fuzzy Soft Mappings 35

3.1 Introduction and preliminaries . . . . . . . . . . . 36

3.2 Fuzzy soft elements . . . . . . . . . . . . . . . . 38

3.3 Fuzzy soft mappings . . . . . . . . . . . . . . . . . . . 42

3.4 Fixed points of fuzzy soft mappings . . . . . . . . . . . . . . . 47

4 Soft Contraction Mappings $\quad 51$

4.1 Introduction and preliminaries . . . . . . . . . . . . 52

4.2 Fixed point theorems . . . . . . . . . . . . . 57

5 Multivalued Caristi's Type Mappings in Fuzzy Metric Spaces and a Characterization of Fuzzy Metric Completeness $\quad 67$

5.1 Introduction and preliminaries . . . . . . . . . . . . 68

5.2 The results . . . . . . . . . . . . . . . . 70 
6 Fixed and Periodic Points of Generalized Contractions in Metric Spaces and in Normed Spaces $\quad \mathbf{7 7}$

6.1 Introduction and preliminaries . . . . . . . . . . . 78

6.2 Common fixed point results in ordered metric spaces . . . . 83

6.3 Periodic points results in metric spaces . . . . . . . . . . . . 89

6.4 Fixed points of generalized contractions . . . . . . . . . . . 92

6.5 Invariant approximation results in non-convex subsets of normed spaces . . . . . . . . . . . . . . . . . . . . . . 99

7 Discussion of the Obtained Results and Conclusions 103

$\begin{array}{ll}\text { Bibliography } & 108\end{array}$ 


\section{Chapter 1}

\section{Introduction. Objectives}

\section{$1.1 \quad$ Background of study}

Mathematical models have been used extensively in real world problems related to engineering, computer sciences, economics, social, natural and medical sciences etc. Because of various uncertainties arising in real world situations, methods of classical mathematics may not be successfully applied to solve them.

Fuzzy set theory has been evolved in mathematics as an important tool (initiated by Zadeh [136]) to resolve the issues of uncertainty and ambiguity. But there are certain limitations and deficiencies pertaining to the parametrization in fuzzy set theory.

To overcome these peculiarities, in 1999, Molodtsov [96] introduced soft sets as a mathematical tool to handle uncertainty associated with real world data based problems. The distinguishing attribute of soft set theory is that unlike probability theory and fuzzy set theory, it does not uphold a precise quantity. This attribute has facilitated applications in decision making, demand analysis, forecasting, information science, mathematics and other disciplines $[40,41,44,55,56,89,97,113,138,141]$. 
A lot of activity has been shown in soft set theory (see [7, 9, 65, 66, 67, 68, $69,81,90,96,130,131,132,133,134])$ since an introduction of the concept of soft sets. Maji et al. [87] introduced some basic algebraic operations on soft sets. They defined equality of two soft sets, subset and super set of soft sets, complement of soft sets, null soft set and absolute soft set with examples. Unfortunately, several basic properties in [87] do not hold true in general, these have been pointed out and improved by Yang [133], Ali et al. [9], and Li [83]. Ali et al. [9] defined some restricted intersection and union, the restricted difference and complement of a soft set. Zhu et al. [139] redefined the intersection, complement, and difference of soft sets and investigated the algebraic properties of these operations along with a known union operation. Their operations on soft sets inherit basic properties of operations on classical sets. With the newly defined operations the union of a soft set and its complement is exactly the whole universal soft set which is not true in general with the previously defined operations. Recently Qin and Hong [104] defined soft equality relations (lower soft equality $\approx_{s}$ and upper soft equality $\approx^{s}$ ) and proved results with already defined (see Ali et al. [9]) operations on union and intersection of soft sets.

Maji et al. [86, 87, 88] elaborated on the theory of soft sets, fuzzy soft sets and intuitionistic fuzzy soft sets and highlighted some of their applications. Some basic operations of fuzzy soft union and intersection and other algebraic properties were studied by Ahmad and Kharal [6]. Babitha and Sunil [17] and Dusmanta Kumar [35] defined soft set relations and fuzzy soft relations and applied the theory to decision making problems. Biwas and Samanta [32] introduced relations on intuitionistic fuzzy soft sets.

The notion of soft topology on a soft set was introduced by Cagman et.al [23] and some basic properties of soft topological spaces were studied (see also, [119]). Fuzzy soft topological spaces were studied by Tridiv [100] and Mahanta [85].

Das and Samanta introduced in [29] the notion of soft real sets, soft real 
numbers and discussed their properties. They also gave applications of these concepts in real life problems. Based on these notions, they introduced in [28] the concept of a soft metric. They showed that soft metric space is also a soft topological space.

A fixed point problem defined by a mapping $f$ and a set $X$ is a problem to find a point $x$ in $X$ such that $f(x)=x$, that is, to find a point in domain of a mapping $f$ which remain invariant under the action of $f$. The solution set of such a problem can be empty, a finite set, infinite set or uncountable finite set. The points in the solution set of this problem are called fixed points of mapping $f$.

In the basic real analysis by using intermediate value theorem we can easily prove that if $I=[a, b]$ is a closed interval of $\mathbb{R}$ then a continuous self mapping $f$ on $I$ has at least one fixed point. Generalizing this simple result, Brouwer proved that if $B$ is a closed ball in $\mathbb{R}^{n}$, then a continuous self mapping on $B$ has at least on fixed point. Brouwer's fixed point theorem is the foundation of topological branch of fixed point theory. All the known proofs of Brouwer's fixed point theorem are based on advanced mathematical methods even in the simple case of $\mathbb{R}^{2}$.

One of the basic and the most widely applied fixed point theorem in all of analysis is "Banach ( or Banach- Cassioppoli ) Contraction principle" due to Banach [18]. It states that if $(X, d)$ is a complete metric space and $f: X \rightarrow X$ satisfies

$$
d(f x, f y) \leq k d(x, y)
$$

for all $x, y \in X$, with $k \in(0,1)$, then $f$ has a unique fixed point. The basic idea of this principle rest in the use of successive approximations to establish the existence and uniqueness of solution of an operator equation $f(x)=x$, particularly it can be employed to prove the existence of solution of differential or integral equations. Banach contraction principle [18] is a simple and powerful result with a wide range of applications, including iterative methods for solving linear, nonlinear, differential, inte- 
gral, and difference equations. Due to its applications in mathematics and other related disciplines (see e.g. [33, 82, 113]), Banach contraction principle has been generalized in many directions (for details one can see $[5,15,16,20,21,27,60,43,98,112,106,125,126,127])$. Banach contraction principle lies at the heart of metric fixed point theory. Over the past two decades the development of fixed point theory in metric spaces has attracted considerable attention due to numerous applications in areas such as variational and linear inequalities, optimization, and approximation theory.

The study of fixed points of mappings satisfying certain contractive conditions has been at the center of vigorous research activity. Extensions of Banach contraction principle have been obtained either by generalizing the domain of the mapping or by extending the contractive condition on the mappings $[15,20,33,60,125]$.

Caristi's fixed point theorem is one of the most useful among these generalizations, which further has been extended and generalized by many authors in several directions (see e.g. [4, 43, 62, 63, 76, 78, 103, 111, 137]). Wardowski [129] introduced a new type of contraction called $F$-contraction and proved a fixed point result in complete metric spaces which in turn generalizes the Banach contraction principle

Meinardus [92] and Brosowski [22] employed fixed point theory to obtain invariant approximation results in normed linear spaces. A number of authors generalized their results (see [52, 57, 61, 99, 121, 122, 124] and references therein). On the other hand, Dotson [34] extended Banach's contraction principle for nonexpansive mappings on star-shaped subsets of Banach spaces and proved Brosowski-Meinardus type theorems on invariant approximations. Khan et al. [77] generalized Dotson's results on star shaped subsets of pnormed spaces.

On the other hand, fixed point theory has developed rapidly in metric spaces endowed with a partial ordering. Fixed point theorems in framework of partially ordered metric spaces are a hybrid of two fundamental princi- 
ples: Banach contraction theorem with contractive condition for comparable elements and selection of an initial point to generate a monotone sequence. Indeed, these results deal with a monotone (either order-preserving or orderreversing) mappings with some restriction such as: for some $x_{0} \in \mathcal{X}$, either $x_{0} \preceq \mathcal{T} x_{0}$ or $\mathcal{T} x_{0} \preceq x_{0}$, where $\mathcal{T}$ is a selfmap on a partially ordered metric space. Existence fixed points in ordered metric spaces has been investigated by Ran and Reurings [107, Theorem 2.1]. They also presented applications of their results to linear and nonlinear equations. Subsequently, Nieto and Rodriguez-Lopez [110] extended the results in [107, Theorem 2.1] for nondecreasing mappings and applied to obtain a unique solution for a first order ordinary differential equation with periodic boundary conditions. Since then, a number of results have been proved in the framework of ordered metric spaces (see $[2,12,13,26,54,72,101,116]$ ). Contractive conditions involving a pair of mappings are further additions to the metric fixed point theory and its applications (see for details $[8,19,66,118]$ ).

Prior to 1968 all work involving fixed points used the Banach contraction principle. In 1968 Kannan [75] proved a fixed point theorem for a map satisfying a contractive condition that did not require continuity at each point. This paper was a genesis for a multitude of fixed point papers over the next two decades (see for example, [109] for a listing and comparison of many of these definitions). A number of these papers dealt with fixed points for more than one map. In some cases commutativity between the maps was required in order to obtain a common fixed point. Sessa [118] coined the term weakly commuting. Jungck [70] generalized the notion of weak commutativity by introducing the concept of compatible maps and then weakly compatible maps [71]. There are examples that show that each of these generalizations of commutativity is a proper extension of the previous definition. Also, Jungck established necessary and sufficient conditions for the existence of common fixed points for commuting mappings

Recently, Wardowski [128] introduced a new notion of soft elements of a 
soft set and established its relation with soft operations and soft objects in soft topological space. Employing the concept of a soft elements, a notion of soft mapping which transforms soft sets into soft sets was introduced. Using these definitions, a fixed point theorem for soft mapping defined on a soft compact Hausdorff topological space was proposed. This paper initiates the study of soft mappings and soft fixed points of such mappings.

The evolution of fuzzy mathematics commenced with an introduction of the notion of fuzzy sets by Zadeh [136] in 1965, as a new way to represent vagueness in every day life. Kramosil and Michalek [80] introduced a notion of fuzzy metric space by using continuous t-norms, which generalizes the concept of probabilistic metric space to fuzzy situation. Moreover George and Veeramani $[46,47]$ modified the concept of a fuzzy metric space introduced by Kramosil and Michalek (see also [38]). They obtained a Hausdorff topology for this kind of fuzzy metric space which has applications in quantum particle physics, particularly in connection with both string and $\epsilon^{\infty}$ theory (see, [37] and references mentioned therein). Recently, Gregori et al. [49] gave applications of fuzzy metrics to color image process and used the concept of fuzzy metric to filter noisy images and in other engineering problems of special interests. Fixed point theory in fuzzy metric spaces has been studied by a number of authors. For a wide survey we refer $[39,30,48,51,84,93,94,95,108,115,120,123,140]$ and the references therein.

The study of fixed point theory for multivalued maps was initiated by Kakutani [73] for finite dimensional spaces. The development of the geometric fixed point theory for multivalued maps was initiated by Nedler [98]. Using the concept of Hausdorff metric, he introduced a notion of multivalued contraction maps and established multivalued contraction principle, which contains the Banach contraction principle as a special case. The study of fixed points for nonexpansive maps using the Hausdorff metric was initiated by Markin [91]. Later, an interesting and rich fixed point theory for such 
maps was developed. The theory of multivalued maps has application in control theory, convex optimization, differential equations and economics.

\subsection{Objectives}

Despite of all the work done in soft set theory so far, it is in developing stage. New concepts are being introduced. It has received much more attention of mathematicians working in classical set theory, which is now rapidly being replaced by soft set theory. Soft topological spaces is a topic under consideration these days. Different topological concepts have been reformulated in soft universe and many more are yet to be done. We have seen through the literature reviewing these topics that there are certain situations in soft set theory that have not been covered properly. The main reason of this gap is that basic operations have not been defined in a way to cover more general aspect of the softness. For the past thirty years, metric fixed point theory has been a flourishing area of research. Although a substantial numbers of results dealing with the existence of fixed point theorems for certain mappings have been proved yet there are many unanswered questions regarding the limits to which the theory may be extended.

The purpose of this work is:

- The study of basic operations first. This work has one to many dimensions. Mainly these are defining, generalizations and applications aspects of basic operations in soft set theory. To highlight the shortcomings in the already existing basic operations in soft set theory, introduce some new basic operations including redefining and generalizing the concepts of null soft set, soft subset, universal soft set, union, intersection and soft elements of a soft set reconsidered in [87, 96, 104, 139] 
- To study the lattice structure on the class of soft sets. This will surely refine the bases for the existing soft topologies and will produce more general results as compared to the existing one.

- To study some basic properties of a fuzzy soft element and fuzzy soft mappings with the help of Cartesian product and relations on fuzzy soft sets and then to initiate the study of fixed point in fuzzy soft set theory.

- Applications in fuzzy soft topology in a sense to improve the work done previously and to extend the research contribution further.

- Metric fixed point theory has not yet been intersected with soft set theory. We will bring some sophistications in this direction as well. We will investigate it as well with the purpose to apply soft set theory in solving functional equations.

- The concept of a soft contraction mapping has not been considered so far. We intend to introduce this concept to initiate the study of fixed point in soft metric spaces and to obtain a soft contraction theorem.

- To provide a characterization of fuzzy metric completeness in the case of continuous t-norms greater than or equal to the Lukasiewicz t-norm.

- To initiate the study of common fixed point theory introducing $F$ - contraction mappings with respect to a self mapping on a complete metric space. We intend to introduce a notion of generalized $F$-contraction mappings to prove a fixed point result for generalized nonexpansive mappings on star shaped subsets of normed linear spaces and to initiate the study of invariant approximations in normed linear spaces for such mappings. 
The organization of the thesis is as follows: This is divided into seven chapters.

Chapter 1 describes the general background, objectives, significance and a scope of the study. It also includes literature review, which is essential for better understanding of present study and for future research work in this direction.

Chapter 2 contains some new concepts which generalize existing comparable notions. The notion of generalized soft equality of two soft sets is given with related results. Moreover we give tolerance or dependence relation on the collection of soft sets and soft lattice structure. Examples are provided to illustrate the concepts and results obtained in this chapter.

In Chapter 3, the concept of a fuzzy soft mappings on a fuzzy soft set is introduced and the study of fixed points of such mappings is initiated. The focus of the exposition is on the fundamental properties of fuzzy soft elements and also on the simplicity of arguments and proofs. Some useful properties of fuzzy soft topological spaces are studied. Examples are provided to explain the newly defined concepts and to illustrate the validity of the results in this chapter.

Chapter 4 deals with the study of fixed point in soft metric spaces. The concept of soft contraction mapping on soft metric spaces is introduced. The central theme of this chapter is a soft contraction theorem. This chapter provides all essential tools to study soft fixed point theory in soft metric spaces .

Chapter 5 concentrates on a fixed point theorem for multivalued mappings of Caristi's type in complete fuzzy metric spaces. In particular the main focus lies on a characterization of fuzzy metric completeness in the case of continuous t-norms greater than or equal to the Lukasiewicz t-norm.

The aim of Chapter 6 is to present the notion of $F$-contractions with respect to a self mapping on a metric space. Employing our definitions some common fixed point results are obtained. Results and concepts presented in 
this chapter are supported with examples. As an application of these results, periodic point results for the $F$-contractions in metric spaces are proved. This chapter also includes an introduction of a generalized $F$-contraction mappings which is then used to obtain a fixed point result for generalized nonexpansive mappings on star shaped subsets of normed linear spaces. Some theorems on invariant approximations in normed linear spaces are deduced.

Chapter 7 deals with the summary of findings, conclusion and contribution to enhance the scope of several comparable existing results in the literature. Some research problems are also proposed which could open new avenues of research in these emerging fields of research.

Each chapter of the thesis contains a section "Introduction and preliminaries" which summarizes the material needed to read the chapter independent of others. 


\section{Chapter 2}

\section{On Generalized Soft Equality and Soft Lattice Structure}

The material of this chapter is an adaptation to the thesis of the content of the paper by Muhajid Abbas, Basit Ali and Salvador Romaguera, "On generalized soft equality and soft lattice structure", which is accepted for publication in the JCR-journal FILOMAT 
The definition of a soft topology is based on the concepts of basic soft set operations and its implications. That is why, the study of soft set operations is vital for mathematicians and computer scientist to develop the theory of soft topological spaces. This is the basic theme of this chapter. In this chapter, we propose some new concepts which generalize existing comparable notions in contemporary literature on soft set theory. We introduce the concept of generalized soft equality ( denoted as $g$-soft equality ) of two soft sets and prove that the so called lower and upper soft equality of two soft sets imply $g$-soft equality but converse does not hold. Moreover we give tolerance or dependence relation on the collection of soft sets and soft lattice structure. Examples are provided to illustrate the concepts and results obtained herein.

We redefine the concepts of null soft set and soft subset of a soft set reconsidered in [139]. We introduce the concept of $g$-null soft set and $g$-soft subset of a soft set and this lead us to give a new and generalized soft equality $\left(g\right.$-soft equality) relation $\approx_{g}$. It is shown that $g$-soft equality relation $\approx_{g}$ is more general than soft equality relations $\approx_{s}$ and $\approx^{s}$ on soft sets given in [104]. We provide examples to show that class of $g$-soft equal sets with respect to $\approx_{g}$ is a more general class.

\subsection{Preliminaries}

We begin with some basic definitions and concepts related to soft sets needed in the sequel.

Let $U$ be a given universe and $E$ a set of parameters. Throughout this chapter, $P(U)$ and $P^{*}(U)$ denote the family of all subsets of $U$, and the family of all nonempty subsets of $U$, respectively.

Definition 2.1.1 [96] If $F$ is a set valued mapping on $A \subset E$ taking values in $P(U)$, then a pair $(F, A)$ is called a soft set over $U$.

A soft set $(F, A)$ can be seen as a parametrized family of subsets of the set $U$. For each $e$ in $A$, the set $F(e)$ in $U$ is called $e$ - approximate element 
of the soft set $(F, A)$.

Moreover, in several places of this chapter a soft set $(F, A)$ will be identified with the set $\{(e, F(e)): e \in A\}$.

Definition 2.1.2 [87] A soft set $(F, A)$ over $U$ is said to be a null soft set over $U$ if $F(e)=\emptyset$ for all $e \in A$.

Definition 2.1.3 [87] Let $(F, A)$ and $(G, B)$ be two soft sets over a common universe $U$, we say that $(F, A)$ is a soft subset of $(G, B)$ or $(G, B)$ is super soft set of $(F, A)$, if $A \subseteq B$, and for all $e \in A, F(e)=G(e)$. We write it as $(F, A) \tilde{\subset}(G, B)$.

Zhu and Wen [139] gave a the following slight modification of Definitions 2.1.1 and 2.1.2 to inherit basic classical set operations in soft set theory.

Definition 2.1.4 [139] If $F$ is a set valued mapping on $A \subset E$ taking values in $P^{*}(U)$, then a pair $(F, A)$ is called a soft set over $U$.

Definition 2.1.5 [139] A soft set $(F, A)$ over $U$ in the sense of [139] is said to be a null soft set denoted by $(\emptyset, \emptyset)$ whenever $A=\emptyset$.

Maji et al. [87] gave definitions 2.1.2 and 2.1.3 which do not inherit the property which reads as follows: "null set is a subset of any other set" in soft set theory (see example 2.1.6 below). Zhu and Wen [139] presented definitions 2.1 .4 and 2.1.5 to incorporate this property.

In the following example we show that a null soft set in the sense of [87] need not be a soft subset of any other soft set. It also shows that Definitions 2.1.4 and 2.1.5 do not cover certain situations arising in soft set theory.

Example 2.1.6 Suppose that $U$ is the set of persons given by

$$
U=\left\{p_{1}, p_{2}, p_{3}, p_{4}, p_{5}, p_{6}\right\}
$$

and

$$
A=\{s, i\}, B=\{i, r\}
$$

where $s, i, r$ stand for susceptible, infectious and recovered persons. The soft set $(F, A)$ describes the specific classes of people with respect to the set $A$, dependent upon their experience with respect to the disease and the 
corresponding approximations $F(s)$ and $F(i)$ with respect to parameter set $A$, are the the sets of susceptible and infected people respectively, given as:

$$
F(s)=\emptyset=F(i)
$$

We denote $(F, A)$ as

$$
(F, A)=\{(s, \emptyset),(i, \emptyset)\} .
$$

The soft set $(G, B)$ describes the specific classes of people with respect to the set $B$, dependent upon their experience with respect to the disease and the corresponding approximations $G(i)$ and $G(r)$ with respect to parameter set $B$, are the the sets of infected and recovered people respectively, given as:

$$
G(i)=\emptyset, G(r)=\left\{p_{1}, p_{2}, p_{3}\right\}
$$

We denote $(G, B)$ as

$$
(G, B)=\left\{(i, \emptyset),\left(r,\left\{p_{1}, p_{2}, p_{3}\right\}\right)\right\} .
$$

Here according to Maji et al. [87], $(F, A)$ is a null soft set but clearly according to them $(F, A)$ is not a null soft subset of $(G, B)$ because $A \nsubseteq B$. That is null soft set is not a soft subset of $(G, B)$. As we mentioned before that Zhu and Wen [139] presented Definitions 2.1.4 and 2.1.5 to remove this shortcoming but according to them $(F, A)$ cannot be regarded as a soft set as $F(s)=F(i)=\emptyset$.

Definition 2.1.7 [87] The union of two soft sets $(F, A)$ and $(G, B)$ over a common universe $U$ is denoted by $(F, A) \widetilde{\cup}(G, B)$ is the soft set $(H, C)$, where $C=A \cup B$ and for all $e \in C,(H, C)$ is defined as

$$
H(e)=\left\{\begin{array}{ll}
F(e), & \text { if } e \in A \backslash B \\
G(e), & \text { if } e \in B \backslash A \\
F(e) \cup G(e) & \text { if } e \in A \cap B
\end{array} .\right.
$$

Definition 2.1.8 [9] The restricted union of two soft sets $(F, A)$ and $(G, B)$ over a common universe $U$ is denoted by $(F, A) \cup_{\mathfrak{R}}(G, B)$ is the soft set $(H, C)$, where $C=A \cap B$ and for all $e \in C, H(e)=F(e) \cup G(e)$. 
Definition 2.1.9 [9] The extended intersection of two soft sets $(F, A)$ and $(G, B)$ over a common universe $U$ is denoted by $(F, A) \sqcap_{\varepsilon}(G, B)$ is the soft set $(H, C)$, where $C=A \cup B$ and for all $e \in C,(H, C)$ is defined as

$$
H(e)=\left\{\begin{array}{ll}
F(e), & \text { if } e \in A \backslash B \\
G(e), & \text { if } e \in B \backslash A \\
F(e) \cap G(e) & \text { if } e \in A \cap B
\end{array} .\right.
$$

Definition 2.1.10 [9] The restricted intersection of two soft sets $(F, A)$ and $(G, B)$ over a common universe $U$ is denoted by $(F, A) \cap(G, B)$ is the soft set $(H, C)$, where $C=A \cap B$ and for all $e \in C, H(e)=F(e) \cap G(e)$.

Definition 2.1.11 [9] The relative complement of a soft set $(F, A)$ over a universe $U$ is denoted by $(F, A)^{r}$ and is defined as $(F, A)^{r}=\left(F^{r}, A\right)$, where $F^{r}(e)=U-F(e)$ for each $e \in A$.

Ali et al. [9] gave the following De Morgan's laws with respect to the relative complement of a soft set in soft set theory.

Theorem 2.1.12 $[9]$ Let $(F, A)$ and $(G, B)$ be two soft sets over a common universe $U$ such that $A \cap B \neq \emptyset$, then

$$
\begin{aligned}
(F, A) \cup_{\mathfrak{R}}(G, B)^{r} & =(F, A)^{r} \cap(G, B)^{r} \\
(F, A) \cap(G, B)^{r} & =(F, A)^{r} \cup_{\mathfrak{R}}(G, B)^{r}
\end{aligned}
$$

holds true.

Recently Qin and Hong [104] defined soft equalities $\approx_{s}$ and $\approx^{s}$. We call these as lower soft equality and upper soft equality relations, respectively.

Definition 2.1.13 [104] Let $(F, A)$ and $(G, B)$ be two soft sets over a common universe $U$. The soft set $(F, A)$ is called lower soft equal to $(G, B)$, denoted by $(F, A) \approx_{s}(G, B)$, if $F(e)=G(e)$ whenever $e \in A \cap B, F(e)=\emptyset$ whenever $e \in A \backslash B$ and $G(e)=\emptyset$ whenever $e \in B \backslash A$.

Definition 2.1.14 [104] Let $(F, A)$ and $(G, B)$ be two soft sets over a common universe $U$. The soft set $(F, A)$ is called upper soft equal to $(G, B)$, denoted by $(F, A) \approx^{s}(G, B)$, if $F(e)=G(e)$, whenever $e \in A \cap B, F(e)=U$ whenever $e \in A \backslash B$ and $G(e)=U$ whenever $e \in B \backslash A$. 
For more on soft equal relations $\approx_{s}$ and $\approx^{s}$, we refer to [104].

\subsection{Generalized soft equality (or $g$-soft equal- ity) relation $\approx_{g}$}

In this section we present a definition of $g$-soft equality of two soft sets which generalizes Definitions 2.1.13 and 2.1.14. We give the concept of a generalized null soft denoted as $g$-null soft set, which unifies definitions 2.1.2 and 2.1.5. A notion of a " $g$-soft subset of a soft set" is also introduced. It is worth mentioning that the definitions presented herein preserve the classical property of a crisp set theory that an empty set is a subset of every set. These notions not only generalize existing comparable concepts but also fit in the bigger set of situations.

Definition 2.2.1 A soft set $(F, A)$ over $U$ is said to be a $g$-null soft set if either $(i) A=\emptyset$ or $(i i) F(e)=\emptyset$ for each $e \in A$ whenever $A \neq \emptyset$. A $g$-null soft set over $U$ is denoted by $\left(F_{\emptyset}, A\right)$.

Definition 2.2.2 A soft set $(F, A)$ over $U$ is called a $g$ - universal soft set if $A=E \neq \emptyset$ and $F(e)=U$ for each $e \in E$. We denote universal soft set by $\left(F_{U}, E\right)$.

Definition 2.2.3 Let $(F, A)$ and $(G, B)$ be two soft sets over a common universe $U$. We say that $(F, A)$ is a $g$-soft subset of $(G, B)$ if for each $e \in A$, there exists an $e^{\prime} \in B$ such that $F(e) \subseteq G\left(e^{\prime}\right)$. We denote it as $(F, A) \sqsubseteq g(G, B)$.

According to Example 2.1.6, $(F, A)$ is a $g$-null soft set and clearly $(F, A) \sqsubseteq g$ $(G, B)$. Hence Definitions 2.2.1 and 2.2.3 inherit the property from classical set theory which says that null set is subset of every other non-empty set.

Example 2.2.4 Suppose that $U=\left\{s_{1}, s_{2}, s_{3}, s_{4}, s_{5}, s_{6}\right\}$ is a set of six students under consideration. Let $s, g$, and $p$ stands for scholarship, good CGPA (we denote good CGPA if CGPA is greater or equal to 3.00 out of 
4.00) and publications, respectively. If the sets of parameters are given as

$$
A=\{s, g\} \text {, and } B=\{s, g, p\} .
$$

Suppose that soft set $(F, A)$ describes the choice of a person P-1 (say) with respect to the parameter set $A$ and soft set $(G, B)$ describes the choice of a person P-2 (say) with respect to the parameter set $B$. Corresponding approximations are given as:

$$
\begin{aligned}
(F, A) & =\left\{\left(s,\left\{s_{1}, s_{2}\right\}\right),\left(g,\left\{s_{2}, s_{5}\right\}\right)\right\} \text { and } \\
(G, B) & =\left\{\left(s,\left\{s_{2}, s_{5}, s_{4}\right\}\right),\left(g,\left\{s_{3}, s_{4}\right\}\right),\left(p,\left\{s_{1}, s_{2}, s_{6}\right\}\right)\right\}
\end{aligned}
$$

where

$$
\begin{aligned}
& F(s)=\left\{s_{1}, s_{2}\right\}, \text { (set of students holding scholarship) } \\
& F(g)=\left\{s_{2}, s_{5}\right\}, \text { (set of students with good CGPA) } \\
& G(s)=\left\{s_{2}, s_{5}, s_{4}\right\}, \text { (set of students holding scholarship) } \\
& G(g)=\left\{s_{3}, s_{4}\right\}, \text { (set of students with good CGPA) } \\
& G(p)=\left\{s_{1}, s_{2}, s_{6}\right\}, \text { (set of students with publications). }
\end{aligned}
$$

Clearly, $(F, A) \sqsubseteq_{g}(G, B)$. That means if according to $\mathrm{P}-1$, a particular student has a certain attribute then that student also exists in the set of P-2's opinion with some attribute (same or different) because $(F, A) \sqsubseteq g(G, B)$. Here according to P-1, students $s_{2}$ and $s_{5}$ have good CGPA and in P-2's opinion, these students hold scholarship as well.

Definition 2.2.5 Let $(F, A)$ and $(G, B)$ be two soft sets over a common universe $U$. Then soft sets $(F, A)$ and $(G, B)$ are called $g$-soft equal if $(F, A) \sqsubseteq_{g}(G, B)$ and $(G, A) \sqsubseteq_{g}(F, B)$. We denote it by $(F, A) \approx_{g}(G, B)$.

In above definition if we take $A \subseteq B$ and $e^{\prime}=e$ then definition 2.2.5 reduces to definition 2.1.3.

Proposition 2.2.6 Let $(F, A)$ and $(G, B)$ be two soft sets over a common universe $U$. If $(F, A) \approx_{s}(G, B)$ then $(F, A) \approx_{g}(G, B)$, that is, lower soft equality implies $g$-soft equality. 
Proof. Suppose that $(F, A) \approx_{s}(G, B)$. Let $e$ be an arbitrary parameter in $A$. There are two possibilities: either $e \in A \backslash B$ or $e \in A \cap B$. If $e \in A \backslash B$, this implies that $F(e)=\emptyset$. So for every $e^{\prime} \in B$, one must have $F(e) \subset G\left(e^{\prime}\right)$ and the result follows. Indeed, if we chose $e^{\prime} \in B \backslash A \subset B$, then $G\left(e^{\prime}\right)=\emptyset$ and $\emptyset=F(e) \subseteq G\left(e^{\prime}\right)=\emptyset$. If $e \in A \cap B$, then by lower soft equality of $(F, A)$ and $(G, B)$, we get $F(e)=G(e)$. Consequently for every $e \in A$, one may finds an $e^{\prime} \in B$ such that $F(e) \subseteq G\left(e^{\prime}\right)$, that is $(F, A) \sqsubseteq g(G, B)$. Now we prove that $(G, B) \sqsubseteq_{g}(F, A)$. For this, let $e \in B$. In this case, either $e \in B \backslash A$ or $e \in A \cap B$. If $e \in B \backslash A$, this implies that $G(e)=\emptyset$. So for every $e^{\prime} \in A$, one must have $G(e) \subset F\left(e^{\prime}\right)$ and the result follows. Indeed, if we chose $e^{\prime} \in$ $A \backslash B \subset A$, then $F\left(e^{\prime}\right)=\emptyset$ and $\emptyset=G(e) \subseteq F\left(e^{\prime}\right)=\emptyset$. If $e \in A \cap B$, then by lower soft equality of $(F, A)$ and $(G, B)$, we get $G(e)=F(e)$. Consequently for every $e \in B$, there exists an $e^{\prime} \in A$ such that $G(e) \subseteq F\left(e^{\prime}\right)$ which implies that $(G, B) \sqsubseteq_{g}(F, A)$. Hence we conclude that $(F, A) \approx_{g}(G, B)$.

Proposition 2.2.7 Let $(F, A)$ and $(G, B)$ be two soft sets over a common universe $U$. If $(F, A) \approx^{s}(G, B)$, then $(F, A) \approx_{g}(G, B)$.

Proof. Following similar arguments to those given in Proposition 2.2.6, the result holds.

Now we give an example to show that if $(F, A) \approx_{g}(G, B)$, then the soft sets $(F, A)$ and $(G, B)$ are not necessarily lower soft equal or upper soft equal. Moreover this example shows that Definition 2.2.5 gives rise to the bigger class of soft subsets of a soft set.

Example 2.2.8 Suppose that $U=\left\{h_{1}, h_{2}, h_{3}, h_{4}\right\}$ is a given universe and $A=\left\{e_{1}, e_{2}, e_{3}\right\}$, and $B=\left\{e_{1}, e_{2}, e_{4}\right\}$ is the set of parameters. Soft sets $(F, A)$ and $(G, A)$ are given as:

$$
\begin{aligned}
(F, A) & =\left\{\left(e_{1},\left\{h_{1}, h_{2}\right\}\right),\left(e_{2},\left\{h_{3}, h_{4}\right\}\right),\left(e_{3}, \emptyset\right)\right\} \text { and } \\
(G, B) & =\left\{\left(e_{1},\left\{h_{3}, h_{4}\right\}\right),\left(e_{2},\left\{h_{1}, h_{2}\right\}\right),\left(e_{4}, \emptyset\right)\right\}
\end{aligned}
$$


where

$$
\begin{aligned}
& F\left(e_{1}\right)=\left\{h_{1}, h_{2}\right\}, F\left(e_{2}\right)=\left\{h_{3}, h_{4}\right\} \text { and } F\left(e_{3}\right)=\emptyset, \\
& G\left(e_{1}\right)=\left\{h_{3}, h_{4}\right\}, G\left(e_{2}\right)=\left\{h_{1}, h_{2}\right\} \text { and } G\left(e_{4}\right)=\emptyset .
\end{aligned}
$$

As $A \nsubseteq B$ and $F\left(e_{1}\right) \nsubseteq G\left(e_{1}\right)$, so by Definition 2.1.3, $(F, A)$ is not a soft subset of $(G, A)$. Note that $F\left(e_{1}\right) \subseteq G\left(e_{2}\right)$ and $F\left(e_{2}\right) \subseteq G\left(e_{1}\right)$. Therefore we have $(F, A) \sqsubseteq_{g}(G, B)$ and $(G, A) \sqsubseteq_{g}(F, B)$ which implies that $(F, A) \approx_{g}$ $(G, B)$. Also, $F\left(e_{1}\right) \neq G\left(e_{1}\right)$ and $F\left(e_{2}\right) \neq G\left(e_{2}\right)$. Therefore, neither $(F, A) \approx_{s}$ $(G, B)$ nor $(F, A) \approx^{s}(G, B)$ hold true. That is, generalized soft equality does not imply lower and upper soft equality.

Proposition 2.2.9 For any soft set $(F, B)$ over $U$

$$
\left(F_{\emptyset}, A\right) \sqsubseteq_{g}(F, B) \sqsubseteq_{g}\left(F_{U}, E\right) .
$$

Proof. If $A=\emptyset$, then assertion holds trivially. Let $A \neq \emptyset$, then for all $e \in A$

$$
F_{\emptyset}(e)=\emptyset \subseteq F\left(e^{\prime}\right) \text { for all } e^{\prime} \in B
$$

and for any $e$ in $B$, we have

$$
F(e) \subseteq U=F_{U}\left(e^{\prime}\right) \text { for all } e^{\prime} \in B
$$

Hence

$$
\left(F_{\emptyset}, A\right) \sqsubseteq_{g}(F, B) \sqsubseteq_{g}\left(F_{U}, E\right)
$$

\subsection{Lattice structure on the soft sets}

In this section, we study soft algebraic operations $\cap, \cup_{\mathfrak{R}}, \widetilde{\cup}, \sqcap_{\varepsilon}$ with reference to $g$-soft equality relation $\approx_{g}$. We also give a lattice structure on a class of soft sets.

Proposition 2.3.1 Let $(F, A)$ be any soft set over $U$, then

(a) $(F, A) \cap\left(F_{U}, E\right) \approx_{g}(F, A)$ 
(b) $(F, A) \cup_{\mathfrak{R}}\left(F_{\emptyset}, A\right) \approx_{g}(F, A)$.

Proof. If $A=\emptyset$, then $(F, A)=\left(F_{\emptyset}, A\right)$ and (a) and (b) hold true. If $A \neq \emptyset$, then $(F, A) \cap\left(F_{U}, E\right)=(H, A \cap E)$. As for each $e$ in $A \cap E(=A)$, we obtain

$$
H(e)=F(e) \cap F_{U}(e)=F(e) \cap U=F(e) .
$$

Therefore (a) follows. Similarly if $(F, A) \cup_{\mathfrak{R}}\left(F_{\emptyset}, A\right)=(K, A)$, then for each $e$ in $A$, we have

$$
K(e)=F(e) \cup F_{\emptyset}(e)=F(e) \cup \emptyset=F(e),
$$

and (b) follows.

Then following theorem shows that the operation $\widetilde{\cup}$ is idempotent, associative and commutative with respect to the $g$-soft equality relation $\approx_{g}$.

Theorem 2.3.2 If $(F, A),(G, B)$ and $(H, C)$ are soft sets over a common universe $U$. Then

(c) $(F, A) \widetilde{\cup}(F, A) \approx_{g}(F, A)$,

(d) $(F, A) \widetilde{\cup}(G, B) \approx_{g}(G, B) \widetilde{\cup}(F, A)$,

(e) $[(F, A) \widetilde{\cup}(G, B)] \widetilde{\cup}(H, C) \approx_{g}(F, A) \widetilde{\cup}[(G, B) \widetilde{\cup}(H, C)]$.

Proof. (c) Let $(F, A) \widetilde{\cup}(F, A)=(K, A)$, then $K(e)=F(e)$, hence (c) follows. It is straightforward to check (d). To prove (e), let

$$
\begin{aligned}
& {[(F, A) \widetilde{\cup}(G, B)] \widetilde{\cup}(H, C)=\left(K_{1}, A \cup B\right) \widetilde{\cup}(H, C)=(K, D) \text { and }} \\
& (F, A) \widetilde{\cup}[(G, B) \widetilde{\cup}(H, C)]=(F, A) \widetilde{\cup}\left(L_{1}, B \cup C\right)=(L, D),
\end{aligned}
$$

where $D=(A \cup B) \cup C=A \cup(B \cup C)$. Let $e \in D$. Obviously $e \in A$, or $e \in B$ or $e \in C$. First suppose that $e \in C$, then the following cases arise:

(e-i) If $e \notin A$ and $e \notin B$, that is $e \in C \backslash(A \cup B)$, then, $K(e)=H(e)$. Moreover $e \in(B \cup C) \backslash A$ implies $L(e)=L_{1}(e)$. As $e \notin B$ and $e \in C$, that is $e \in C \backslash B$, so $L_{1}(e)=H(e)$. Consequently $K(e)=L(e)$. 
(e-ii) If $e \in A$ and $e \notin B$, then $e \in A \backslash B$, this implies $K_{1}(e)=F(e)$ and $e \in A \cup B$ and $e \in C$ implies $K(e)=K_{1}(e) \cup H(e)=F(e) \cup H(e)$. Moreover, if $e \in A$ and $e \notin B$, then $e \in C \backslash B$, this implies $L_{1}(e)=H(e)$. Since $e \in A$ and $e \in B \cup C$, we obtain $L(e)=F(e) \cup L_{1}(e)=F(e) \cup H(e)$. Consequently $K(e)=L(e)$.

(e-iii) If $e \notin A$ and $e \in B$, then $e \in B \backslash A$ implies that $K_{1}(e)=G(e)$. If $e \in A \cup B$ and $e \in C$, then we have $K(e)=K_{1}(e) \cup H(e)=G(e) \cup H(e)$. Since $e \in B \cap C$, this gives $L_{1}(e)=G(e) \cup H(e)$. Further $e \notin A$ and $e \in B \cup C$, implies $L(e)=L_{1}(e)=G(e) \cup H(e)$. Consequently $K(e)=L(e)$.

(e-vi) If $e \in A$ and $e \in B$, that is, $e \in A \cap B$, this implies $K_{1}(e)=$ $F(e) \cup G(e)$. Also, $e \in A \cup B$ and $e \in C$ implies $K(e)=K_{1}(e) \cup H(e)=$ $F(e) \cup G(e) \cup H(e)$. As $e \in B$ and $e \in C$, so $e \in B \cap C$, this implies $L_{1}(e)=G(e) \cup H(e)$. Since $e \in A$ and $e \in B \cup C$, we obtain $L(e)=F(e) \cup$ $L_{1}(e)=F(e) \cup G(e) \cup H(e)$. Consequently $K(e)=L(e)$.

Following arguments similar to those given in (e-i) to (e-iv), the result follows in each of the case when $e \in B$ and $e \in A$. Since for all $e \in D, K$ and $L$ are the same approximations, so we conclude that

$$
[(F, A) \widetilde{\cup}(G, B)] \widetilde{\cup}(H, C) \approx_{g}(F, A) \widetilde{\cup}[(G, B) \widetilde{\cup}(H, C)] .
$$

Theorem 2.3.3 Let $(F, A),(G, B)$ and $(H, C)$ be soft sets over a common universe $U$. Then

(f) $(F, A) \sqcap_{\varepsilon}(F, A) \approx_{g}(F, A)$

(g) $(F, A) \sqcap_{\varepsilon}(G, B) \approx_{g}(G, B) \sqcap_{\varepsilon}(F, A)$,

(h) $\left[(F, A) \sqcap_{\varepsilon}(G, B)\right] \sqcap_{\varepsilon}(H, C) \approx_{g}(F, A) \sqcap_{\varepsilon}\left[(G, B) \sqcap_{\varepsilon}(H, C)\right]$.

Proof. (f) Let $(F, A) \sqcap_{\varepsilon}(F, A)=(K, A)$, then $K(e)=F(e)$, hence (f) follows. (g) is straightforward to check. To prove (h), let

$$
\begin{aligned}
{\left[(F, A) \sqcap_{\varepsilon}(G, B)\right] \sqcap_{\varepsilon}(H, C) } & =\left(K_{1}, A \cup B\right) \sqcap_{\varepsilon}(H, C)=(K, D) \text { and } \\
(F, A) \sqcap_{\varepsilon}\left[(G, B) \sqcap_{\varepsilon}(H, C)\right] & =(F, A) \sqcap_{\varepsilon}\left(L_{1}, B \cup C\right)=(L, D),
\end{aligned}
$$


where $D=A \cup B \cup C$. Let $e \in D$. Obviously $e \in A$, or $e \in B$ or $e \in \dot{C}$. First suppose that $e \in \dot{C}$, then there arise following cases:

(e-i). If $e \notin A$ and $e \notin B$, that is $e \in C \backslash(A \cup B)$, then, $K(e)=H(e)$. Moreover $e \in(B \cup C) \backslash A$ implies $L(e)=L_{1}(e)$. As $e \notin B$ and $e \in C$, that is $e \in C \backslash B$, so $L_{1}(e)=H(e)$. Consequently $K(e)=L(e)$.

(eii). If $e \in A$ and $e \notin B$, then $e \in A \backslash B$, this implies $K_{1}(e)=F(e)$ and $e \in A \cup B$ and $e \in C$ implies $K(e)=K_{1}(e) \cap H(e)=F(e) \cap H(e)$. Moreover, if $e \in A$ and $e \notin B$, then $e \in C \backslash B$, this implies $L_{1}(e)=H(e)$. Since $e \in A$ and $e \in B \cup C$, we obtain $L(e)=F(e) \cap L_{1}(e)=F(e) \cap H(e)$. Consequently $K(e)=L(e)$.

(e-iii). If $e \notin A$ and $e \in B$, then $e \in B \backslash A$, this implies $K_{1}(e)=G(e)$ and $e \in A \cup B$ and $e \in C$ implies $K(e)=K_{1}(e) \cap H(e)=G(e) \cap H(e)$. Since $e \in B \cap C$, this gives $L_{1}(e)=G(e) \cap H(e)$. Further $e \notin A$ and $e \in B \cup C$, implies $L(e)=L_{1}(e)=G(e) \cap H(e)$. Consequently $K(e)=L(e)$.

(e-iv). If $e \in A$ and $e \in B$, that is, $e \in A \cap B$, this implies $K_{1}(e)=$ $F(e) \cap G(e)$. Also, $e \in A \cup B$ and $e \in C$ implies $K(e)=K_{1}(e) \cap H(e)=$ $F(e) \cap G(e) \cap H(e)$. As $e \in B$ and $e \in C$, so $e \in B \cap C$, this implies $L_{1}(e)=G(e) \cap H(e)$. Since $e \in A$ and $e \in B \cup C$, we obtain $L(e)=F(e) \cap$ $L_{1}(e)=F(e) \cap G(e) \cap H(e)$. Consequently $K(e)=L(e)$.

Following arguments similar to those given in (e-i) to (e-iv), the result follows in each of the case when $e \in B$ and $e \in A$. Since for all $e \in D, K$ and $L$ are the same approximations, so we conclude that

$$
\left[(F, A) \sqcap_{\varepsilon}(G, B)\right] \sqcap_{\varepsilon}(H, C) \approx_{g}(F, A) \sqcap_{\varepsilon}\left[(G, B) \sqcap_{\varepsilon}(H, C)\right] .
$$

Note that Theorem 2.3.2 and Theorem 2.3.3 hold for the operations $\cup_{\Re}$, and $\cap$ as well.

Following is an absorption law involving operations $\widetilde{U}$, ก and $g$-soft equality.

Theorem 2.3.4 Let $(F, A),(G, B)$ and $(H, C)$ be soft sets over a common universe $U$. Then 
(i) $[(F, A) \widetilde{\cup}(G, B)] \cap(F, A) \approx_{g}(F, A)$.

(j) $[(F, A) \cap(G, B)] \widetilde{\cup}(F, A) \approx_{g}(F, A)$.

Proof. Suppose

$[(F, A) \widetilde{\cup}(G, B)] \cap(F, A)=(H, A \cup B) \cap(F, A)=(K,(A \cup B) \cap A)=(K, A)$

So soft sets on both sides of (i) have the same parameter set $A$. Let $e$ be an arbitrary element of $A$. if $e \notin B$, then $H(e)=F(e)=H(e) \cap F(e)=K(e)$. If $e \in B$, then $H(e)=F(e) \cup G(e)$ and $F(e) \subseteq H(e)$ which further implies that $F(e) \subseteq H(e) \cap F(e)=K(e)$, that is, $F(e) \subseteq K(e)$. Hence $(F, A) \sqsubseteq g(K, A)$. On the other hand

$$
K(e)=H(e) \cap F(e)=[F(e) \cup G(e)] \cap F(e)=F(e) \subseteq F(e),
$$

implies that $(K, A) \sqsubseteq_{g}(F, A)$. Consequently $(K, A) \approx_{g}(F, A)$. Similarly, it can be shown that $(\mathrm{j})$ holds true.

In the following theorem, we show that $\widetilde{\cup}$ is distributive over $\cap$.

Theorem 2.3.5 Let $(F, A),(G, B)$ and $(H, C)$ be soft sets over a common universe $U$. Then

$$
(F, A) \widetilde{\cup}[(G, B)) \cap(H, C)] \widetilde{\simeq}_{g}[(F, A) \widetilde{\cup}((G, B)] \cap[(F, A) \widetilde{\cup}(H, C)] .
$$

\section{Proof. Suppose}

$(F, A) \widetilde{\cup}[(G, B)) \cap(H, C)]=(F, A) \widetilde{\cup}\left(K_{1},(B \cap C)\right)=(K, A \cup(B \cap C))=(K, D)$, and

$\left[(F, A) \widetilde{\cup}((G, B)] \cap[(F, A) \widetilde{\cup}(H, C)]=\left(L_{1},(A \cup B)\right) \cap\left(L_{2},(A \cup C)\right)=(L, D)\right.$, where $D=A \cup(B \cap C)=(A \cup B) \cap(A \cup C)$. Now for all $e \in D$, it follows that $e \in A$ or $e \in B$ and $e \in A$ or $e \in \dot{C}$. First suppose that $e \in \dot{C}$, then there arise following cases: 
(k-i). If $e \in A$ and $e \notin B$, that is, $e \in A \backslash(B \cap C)$, then $K(e)=F(e)$. Since $e \in A \backslash B$ and $e \in A \cap C$, so $L_{1}(e)=F(e)$ and $L_{2}(e)=F(e) \cup H(e)$. Hence $L(e)=L_{1}(e) \cap L_{2}(e)=F(e)$. Consequently $K(e)=L(e)$.

(k-ii). If $e \notin A$ and $e \in B$, that is, $e \in(B \cap C) \backslash A$, then $K(e)=K_{1}(e)=$ $G(e) \cap H(e)$. Moreover, $e \in B \backslash A$ and $e \in C \backslash A$, implies that $L_{1}(e)=G(e)$ and $L_{2}(e)=H(e)$. Hence $L(e)=L_{1}(e) \cap L_{2}(e)=G(e) \cap H(e)$. Consequently $K(e)=L(e)$.

(k-iii). If $e \in A$ and $e \in B$, then $e \in A$ and $e \in(B \cap C)$ implies that

$$
K(e)=F(e) \cup K_{1}(e)=F(e) \cup[G(e) \cap H(e)]
$$

Since $e \in A \cap B$ and $e \in A \cap C$, so $L_{1}(e)=F(e) \cup G(e)$ and $L_{2}(e)=$ $F(e) \cup H(e)$. Hence

$$
L(e)=L_{1}(e) \cap L_{2}(e)=F(e) \cup[G(e) \cap H(e)] .
$$

Consequently $K(e)=L(e)$.

The cases (k-i to k-iii) can be discussed for $e \in B$ and $e \in A$. Since for all $e \in A \cup(B \cap C), K$ and $L$ are the same approximations, so we conclude that

$$
(F, A) \widetilde{\cup}[(G, B)) \cap(H, C)] \approx_{g}[(F, A) \widetilde{\cup}((G, B)] \cap[(F, A) \widetilde{\cup}(H, C)] .
$$

Suppose that $S(U, E)$ denotes the set of all soft sets over the common universe $U$ and the parameter set $E$, that is,

$$
S(U, E)=\{(F, A): A \subseteq E \text { and } F: A \rightarrow P(U)\}
$$

Remark 2.3.6 Let $(F, A),(G, B) \in S(U, E)$. If $(F, A) \approx_{g}(G, B)$, then $(F, A) \cap(G, B) \approx_{g}(F, A)$ and $(F, A) \widetilde{\cup}(G, B) \approx_{g}(G, B)$ do not hold true necessarily. See the following example.

Example 2.3.7 Let $U=\left\{h_{1}, h_{2}\right\}$ be a universe under consideration and $A=B=\left\{e_{1}, e_{2}\right\}$ is the set of parameters. Soft sets $(F, A),(G, B)$ are given 
as:

$$
\begin{aligned}
(F, A) & =\left\{\left(e_{1},\left\{h_{1}\right\}\right),\left(e_{2},\left\{h_{2}\right\}\right)\right\} \text { and } \\
(G, B) & =\left\{\left(e_{1},\left\{h_{2}\right\}\right),\left(e_{2},\left\{h_{1}\right\}\right)\right\}
\end{aligned}
$$

Clearly $(F, A) \approx_{g}(G, B)$. If $(F, A) \cap(G, B)=(H, A \cap B)$ and $(F, A) \widetilde{\cup}(G, B)=$ $(K, A \cup B)$, then

$$
\begin{aligned}
(H, A \cap B) & =\left\{\left(e_{1}, \emptyset\right),\left(e_{2}, \emptyset\right)\right\} \text { and } \\
(K, A \cap B) & =\left\{\left(e_{1},\left\{h_{1}, h_{2}\right\}\right),\left(e_{2},\left\{h_{1}, h_{2}\right\}\right)\right\}
\end{aligned}
$$

Clearly $(F, A) \cap(G, B) \not_{g}(F, A)$ and $(F, A) \widetilde{\cup}(G, B) \not_{g}(G, B)$.

Now we define soft ordering relation, denoted by $\preceq_{s}$ on $S(U, E)$. We say that $(F, A) \preceq_{s}(G, B)$ if and only if

$$
(F, A) \cap(G, B) \approx_{g}(F, A) \text { and }(F, A) \widetilde{\cup}(G, B) \approx_{g}(G, B) .
$$

Remark 2.3.8 Following example illustrates the fact that $(F, A) \cap(G, B) \approx_{g}$ $(F, A)$ does not always imply $(F, A) \widetilde{\cup}(G, B) \approx_{g}(G, B)$.

Example 2.3.9 Suppose that $U=\left\{h_{1}, h_{2}, h_{3}, h_{4}\right\}$ and $A=B=\left\{e_{1}, e_{2}\right\}$. Soft sets $(F, A)$ and $(G, A)$ are given as:

$$
\begin{aligned}
(F, A) & =\left\{\left(e_{1},\left\{h_{3}\right\}\right),\left(e_{2},\left\{h_{2}, h_{3}\right\}\right)\right\} \text { and } \\
(G, B) & =\left\{\left(e_{1},\left\{h_{1}, h_{2}\right\}\right),\left(e_{2},\left\{h_{2}, h_{3}\right\}\right)\right\}
\end{aligned}
$$

Suppose that $(F, A) \cap(G, B)=(H, A \cap B)$ and $(F, A) \widetilde{\cup}(G, B)=(K, A \cup B)$. Note that

$$
\begin{aligned}
& (H, A \cap B)=\left\{\left(e_{1}, \emptyset\right),\left(e_{2},\left\{h_{2}, h_{3}\right\}\right)\right\} \text { and } \\
& (K, A \cup B)=\left\{\left(e_{1},\left\{h_{1}, h_{2}, h_{3}\right\}\right),\left(e_{2},\left\{h_{2}, h_{3}\right\}\right)\right\} .
\end{aligned}
$$

Clearly $(F, A) \cap(G, B) \approx_{g}(F, A)$ but $(F, A) \widetilde{\cup}(G, B) \not_{g}(G, B)$ because $K\left(e_{1}\right) \nsubseteq G(e)$ for any $e \in B$.

Theorem 2.3.10 $\left(S(U, E), \widetilde{U}, \cap, \widetilde{\approx}_{g}\right)$ is a distributive bounded lattice. 
Proof. From Theorems 2.3.2, 2.3.3, 2.3.4 and 2.3.5, it follows that $\left(S(U, E), \widetilde{\cup}, \cap, \approx_{g}\right)$ is a distributive lattice. As $\left(F_{\emptyset}, \emptyset\right)$ and $\left(F_{U}, E\right)$ are lower and upper bounds of $S(U, E)$, respectively so $S(U, E)$ is a bounded lattice.

Note that $\left(S(U, E), \cup_{\mathfrak{R}}, \sqcap_{\varepsilon}, \widetilde{\approx}_{g}\right)$ is also a distributive bounded lattice. Let $A \subseteq E$ and

$$
S_{A}(U, E)=\{(F, A): F: A \rightarrow P(U)\}
$$

be the set of all soft sets with parameter set $A$ over a universe $U$. Then we have the following corollary.

Corollary 2.3.11 $\left(S_{A}(U, E), \widetilde{\cup}, \cap, \widetilde{\simeq}_{g}\right)$ is a sublattice of $\left(S(U, E), \widetilde{\cup}, \cap, \widetilde{\simeq}_{g}\right)$. Proposition 2.3.12 The soft ordering relation $\preceq_{s}$ is a tolerance relation on $S(U, E)$ ( $\preceq_{s}$ is reflexive and symmetric).

Proof. Let $(F, A),(G, B)$ and $(H, C)$ be arbitrary elements of $S(U, E)$. Note that $(F, A) \preceq_{s}(F, A)$, that is, $\preceq_{s}$ is reflexive. Now $(F, A) \preceq_{s}(G, B)$ implies that

$$
(F, A) \cap(G, B) \approx_{g}(F, A) \text { and }(F, A) \widetilde{\cup}(G, B) \approx_{g}(G, B)
$$

and $(G, B) \preceq_{s}(F, A)$ implies that

$$
(G, B) \cap(F, A) \approx_{g}(G, B) \text { and }(G, B) \widetilde{\cup}(F, A) \approx_{g}(F, A) .
$$

As $\cap$ and $\widetilde{\cup}$ are commutative so $(F, A) \approx_{g}(G, B)$, that is $\preceq_{s}$ is symmetric.

Following example shows that $\preceq_{s}$ is not a transitive relation.

Example 2.3.13 Suppose that $U=\left\{h_{1}, h_{2}, h_{3}, h_{4}\right\}$ and $A=B=\left\{e_{1}, e_{2}\right\}$. Let soft sets $(F, A),(G, A)$, and $(H, A)$ be given by:

$$
\begin{aligned}
(F, A) & =\left\{\left(e_{1},\left\{h_{1}\right\}\right),\left(e_{2},\left\{h_{2}\right\}\right)\right\} \\
(G, A) & =\left\{\left(e_{1},\left\{h_{1}, h_{2}\right\}\right),\left(e_{2},\left\{h_{1}, h_{2}\right\}\right)\right\} \text { and } \\
(H, A) & =\left\{\left(e_{1},\left\{h_{3}\right\}\right),\left(e_{2},\left\{h_{1}, h_{2}, h_{3}\right\}\right)\right\} .
\end{aligned}
$$

Let $(F, A) \cap(G, A)=(J, A),(F, A) \widetilde{\cup}(G, A)=(K, A),(G, A) \cap(H, A)=$ 
$(L, A)$ and $(G, A) \widetilde{\cup}(H, A)=(M, A)$. Note that

$$
\begin{aligned}
(J, A) & =\left\{\left(e_{1},\left\{h_{1}\right\}\right),\left(e_{2},\left\{h_{2}\right\}\right)\right\} \\
(K, A) & =\left\{\left(e_{1},\left\{h_{1}, h_{2}\right\}\right),\left(e_{2},\left\{h_{1}, h_{2}\right\}\right)\right\} \\
(L, A) & =\left\{\left(e_{1}, \emptyset\right),\left(e_{2},\left\{h_{1}, h_{2}\right\}\right)\right\} \text { and } \\
(M, A) & =\left\{\left(e_{1},\left\{h_{1}, h_{2}, h_{3}\right\}\right),\left(e_{2},\left\{h_{1}, h_{2}, h_{3}\right\}\right)\right\} .
\end{aligned}
$$

Clearly $(J, A) \widetilde{\simeq}_{g}(F, A)$ and $(K, A) \widetilde{\simeq}_{g}(G, A)$ imply that $(F, A) \preceq_{s}(G, A)$. Moreover $(L, A) \approx_{g}(G, A)$ and $(M, A) \approx_{g}(H, A)$ implies that $(G, A) \preceq_{s}$ $(H, A)$. Suppose that $(F, A) \cap(H, A)=\left(N_{1}, A\right)$ and $(F, A) \widetilde{\cup}(H, A)=\left(N_{2}, A\right)$. Note that

$$
\begin{aligned}
& \left(N_{1}, A\right)=\left\{\left(e_{1}, \emptyset\right),\left(e_{2},\left\{h_{2}\right\}\right)\right\} \text { and } \\
& \left(N_{2}, A\right)=\left\{\left(e_{1},\left\{h_{1}, h_{3}\right\}\right),\left(e_{2},\left\{h_{1}, h_{2}, h_{3}\right\}\right)\right\} .
\end{aligned}
$$

Since $F\left(e_{1}\right) \nsubseteq N_{1}(e)$ for any $e \in A$, therefore $(F, A) \nsubseteq_{g}(F, A) \cap(H, A)$. This implies that $(F, A) \not_{g}(F, A) \cap(H, A)$. Hence $(F, A) \preceq_{s}(G, A)$ and $(G, A) \preceq_{s}(H, A)$ but $(F, A) \swarrow_{s}(H, A)$.

Proposition 2.3.14 $\approx_{g}$ is an equivalence relation on $S(U, E)$.

Proof. Let $(F, A),(G, B)$ and $(H, C)$ be arbitrary elements of $S(U, E)$. Then by definition $(F, A) \approx_{g}(F, A)$, hence $\approx_{g}$ is reflexive. Also, $(F, A) \approx_{g}$ $(G, B)$ implies that $(G, B) \approx_{g}(F, A)$, that is $\approx_{g}$ is symmetric. Suppose that $(F, A) \approx_{g}(G, B)$ and $(G, B) \approx_{g}(H, C)$. Note that for any $e \in A$ there exists an $e^{\prime} \in B$ such that $F(e) \subseteq G\left(e^{\prime}\right)$ and for $e^{\prime} \in B$ there exists $e^{\prime} \in C$ such that $G\left(e^{\prime}\right) \subseteq H\left(e^{\prime}\right)$. Hence for every $e$ in $A$ there is $e^{\prime}$ in $C$ such that $F(e) \subseteq H\left(e^{\prime}\right)$, thus $(F, A) \sqsubseteq g(H, C)$. Following similar arguments, we have $(H, C) \sqsubseteq_{g}(F, A)$. Hence $(F, A) \approx_{g}(H, C)$.

From Definition 2.1.11, it follows that for any soft set $(F, A),\left((F, A)^{r}\right)^{r}=$ $(F, A)$ holds. Also, De Morgan's laws hold in soft set theory employing the concept of a $g$-soft equality relation $\approx_{g}$.

Theorem 2.3.15 Let $(F, A)$, and $(G, B)$ be soft sets over a common universe $U$ such that $A \cap B \neq \emptyset$, then 
(1) $\left((F, A) \cup_{\mathfrak{R}}(G, B)\right)^{r} \approx_{g}(F, A)^{r} \cap(G, B)^{r}$.

(2) $((F, A) \cap(G, B))^{r} \approx_{g}(F, A)^{r} \cup_{\mathfrak{R}}(G, B)^{r}$.

Proof. Suppose that

$$
\begin{aligned}
(F, A) \cup_{\mathfrak{R}}(G, B) & =(H, A \cap B), \\
\left((F, A) \cup_{\mathfrak{R}}(G, B)\right)^{r} & =\left(H^{r}, A \cap B\right), \\
(F, A)^{r} \cap(G, B)^{r} & =(K, A \cap B),
\end{aligned}
$$

Now for $e \in A \cap B$, we have

$$
H^{r}(e)=U-H(e)=U-[F(e) \cup G(e)]=(U-F(e)) \cap(U-G(e))=K(e) .
$$

Since for all $e \in A \cap B, H^{r}$ and $K$ are same approximations, so we conclude that

$$
\left((F, A) \cup_{\mathfrak{R}}(G, B)\right)^{r} \approx_{g}(F, A)^{r} \cap(G, B)^{r} .
$$

Now from (1), we obtain that

$$
\left((F, A)^{r} \cup_{\mathfrak{R}}(G, B)^{r}\right)^{r} \approx_{g}\left((F, A)^{r}\right)^{r} \cap\left((G, B)^{r}\right)^{r} \approx_{g}(F, A) \cap(G, B) .
$$

Hence

$$
(F, A)^{r} \cup_{\mathfrak{R}}(G, B)^{r} \approx_{g}((F, A) \cap(G, B))^{r} .
$$

Theorem 2.3.16 Let $(F, A)$, and $(G, B)$ be soft sets over a common universe $U$ such that $A \cap B \neq \emptyset$, then

(1) $((F, A) \widetilde{\cup}(G, B))^{r} \approx_{g}(F, A)^{r} \sqcap_{\varepsilon}(G, B)^{r}$.

(2) $\left((F, A) \sqcap_{\varepsilon}(G, B)\right)^{r} \approx_{g}(F, A)^{r} \widetilde{\cup}(G, B)^{r}$.

Proof. Suppose that

$$
\begin{aligned}
(F, A) \widetilde{\cup}(G, B) & =(H, A \cup B), \\
((F, A) \widetilde{\cup}(G, B))^{r} & =\left(H^{r}, A \cup B\right), \text { and } \\
(F, A)^{r} \sqcap_{\varepsilon}(G, B)^{r} & =(K, A \cup B) .
\end{aligned}
$$


Now for $e \in A \cup B$, if $e \in A$ and $e \notin B$, then $H^{r}(e)=U-H(e)=U-F(e)$ and $K(e)=F^{r}(e)=U-F(e)$. If $e \notin A$ and $e \in B$, then $H^{r}(e)=U-H(e)=$ $U-G(e)$ and $K(e)=G^{r}(e)=U-G(e)$. If $e \in A$ and $e \in B$, then

$$
H^{r}(e)=U-H(e)=U-[F(e) \cup G(e)]=[U-F(e)) \cap(U-G(e)]
$$

and

$$
K(e)=F^{r}(e) \cap G^{r}(e)=[U-F(e)) \cap(U-G(e)] .
$$

Hence

$$
\left(H^{r}, A \cup B\right)=(K, A \cup B)
$$

As for all $e \in A \cup B, H^{r}$ and $K$ are the same approximations, so we conclude that

$$
((F, A) \widetilde{\cup}(G, B))^{r} \approx_{g}(F, A)^{r} \sqcap_{\varepsilon}(G, B)^{r}
$$

Now from (1) we obtain

$$
\left((F, A)^{r} \widetilde{\cup}(G, B)^{r}\right)^{r} \approx_{g}\left((F, A)^{r}\right)^{r} \sqcap_{\varepsilon}\left((G, B)^{r}\right)^{r} \approx_{g}(F, A) \sqcap_{\varepsilon}(G, B) .
$$

Hence

$$
(F, A)^{r} \widetilde{\cup}(G, B)^{r} \approx_{g}\left((F, A) \sqcap_{\varepsilon}(G, B)\right)^{r} .
$$

Theorem 2.3.17 [104, Theorem 24-26] Let $(F, A)$ and $(G, B)$ be two soft sets over a common universe $U$. Then $(F, A) \approx_{s}(G, B)$ if and only if

(a) $(F, A) \widetilde{\cup}(G, B) \approx_{s}(F, A) \cap(G, B)$,

(b) $(F, A) \widetilde{\cup}(G, B) \approx_{s}(F, A) \sqcap_{\varepsilon}(G, B)$,

(c) $(F, A) \cup_{\mathfrak{R}}(G, B) \approx_{s}(F, A) \cap(G, B)$,

(d) $(F, A) \cup_{\mathfrak{R}}(G, B) \approx_{s}(F, A) \sqcap_{\varepsilon}(G, B)$.

Theorem 2.3.17 does not hold if we replace $\approx_{s}$ with soft equality $\approx_{g}$. Following example illustrates the fact. 
Example 2.3.18 Suppose that $U=\left\{h_{1}, h_{2}, h_{3}, h_{4}\right\}$ and $A=\left\{e_{1}, e_{2}\right\}$. Soft sets $(F, A)$ and $(G, A)$ are given as:

$$
\begin{aligned}
& (F, A)=\left\{\left(e_{1},\left\{h_{1}, h_{2}\right\}\right),\left(e_{2},\left\{h_{3}, h_{4}\right\}\right)\right\} \text { and } \\
& (G, A)=\left\{\left(e_{1},\left\{h_{3}, h_{4}\right\}\right),\left(e_{2},\left\{h_{1}, h_{2}\right\}\right)\right\} .
\end{aligned}
$$

Clearly $F\left(e_{1}\right) \cup G\left(e_{1}\right)=F\left(e_{2}\right) \cup G\left(e_{2}\right)=U$ and $F\left(e_{1}\right) \cap G\left(e_{1}\right)=F\left(e_{2}\right) \cap$ $G\left(e_{2}\right)=\emptyset$. Hence

$$
\begin{aligned}
(F, A) \widetilde{\cup}(G, A) & \not{z}{ }_{g}(F, A) \cap(G, A), \\
(F, A) \widetilde{\cup}(G, A) & \not{z}{ }_{g}(F, A) \sqcap_{\varepsilon}(G, A), \\
(F, A) \cup_{\mathfrak{R}}(G, A) & \not{z}{ }_{g}(F, A) \cap(G, A), \\
(F, A) \cup_{\mathfrak{R}}(G, A) & \not{z}{ }_{g}(F, A) \sqcap_{\varepsilon}(G, A) .
\end{aligned}
$$

Remark 2.3.19 The lower soft equality relation $\approx_{s}$ is a congruence relation ( $\left[104\right.$, Theorem 28]) that is $(F, A) \approx_{s}(G, A)$ and $(H, A) \approx_{s}(I, A)$ imply that $(F, A) \cap(H, A) \approx_{s}(G, A) \cap(I, A)$ and $(F, A) \widetilde{\cup}(H, A) \approx_{s}(G, A) \widetilde{\cup}(I, A)$, while $\approx_{g}$ is not a congruence relation. To see it consider the following example.

Example 2.3.20 Suppose that $U=\left\{h_{1}, h_{2}, h_{3}, h_{4}\right\}$ and $A=\left\{e_{1}, e_{2}\right\}$. Soft sets $(F, A),(G, A),(H, A)$ and $(I, A)$ are given as:

$$
\begin{aligned}
(F, A) & =\left\{\left(e_{1}, U\right),\left(e_{2},\left\{h_{1}, h_{3}\right\}\right)\right\} \\
(G, A) & =\left\{\left(e_{1},\left\{h_{2}, h_{4}\right\}\right),\left(e_{2}, U\right)\right\} \\
(H, A) & =\left\{\left(e_{1},\left\{h_{1}, h_{2}\right\}\right),\left(e_{2},\left\{h_{3}, h_{4}\right\}\right)\right\}, \text { and } \\
(I, A) & =\left\{\left(e_{1},\left\{h_{3}, h_{4}\right\}\right),\left(e_{2},\left\{h_{1}, h_{2}\right\}\right)\right\} .
\end{aligned}
$$

Clearly $(F, A) \approx_{g}(G, A)$ and $(H, A) \approx_{g}(I, A)$. Now let $(F, A) \cap(H, A)=$ $(J, A),(G, A) \cap(I, A)=(K, A),(F, A) \widetilde{\cup}(H, A)=(L, A)$ and $(G, A) \widetilde{\cup}(I, A)=$ $(M, A)$. Now

$$
\begin{aligned}
(J, A) & =\left\{\left(e_{1},\left\{h_{1}, h_{2}\right\}\right),\left(e_{2},\left\{h_{3}\right\}\right)\right\}, \\
(K, A) & =\left\{\left(e_{1},\left\{h_{4}\right\}\right),\left(e_{2},\left\{h_{1}, h_{2}\right\}\right)\right\}, \\
(L, A) & =\left\{\left(e_{1}, U\right),\left(e_{2},\left\{h_{1}, h_{3}, h_{4}\right\}\right)\right\}, \text { and } \\
(M, A) & =\left\{\left(e_{1},\left\{h_{2}, h_{3}, h_{4}\right\}\right),\left(e_{2}, U\right)\right\} .
\end{aligned}
$$


Note that $(J, A) \varpi_{g}(K, A)$ and $(L, A) \varpi_{g}(M, A)$. Hence $(F, A)$ ก $(H, A) \varpi_{g}$ $(G, A) \cap(I, A)$ and $(F, A) \widetilde{\cup}(H, A) \not_{g}(G, A) \widetilde{\cup}(I, A)$. Consequently $\approx_{g}$ is not a congruence relation. 


\section{Chapter 3}

\section{Fixed Points of Fuzzy Soft Mappings}

The material of this chapter is an adaptation to the thesis of the content of the paper by Muhajid Abbas, Asma Khalid and Salvador Romaguera, "Fixed points of fuzzy soft mappings", published in the JCR-journal Applied Mathematics and Information Sciences 8 (2014), 2141-2147. 
Wardowski [128] introduced a notion of soft mappings and obtained a fixed point result for a fixed point of a soft mapping in soft compact Hausdorff topological spaces. He also studied the properties of soft compact topological spaces. His main result is based on the fact that a decreasing sequence of nonempty soft closed subsets in soft compact topological spaces has a nonempty intersection.

Roy and Samanta [114] introduced the concept of a fuzzy soft topology and studied some of its basic properties. Neog et al. [100], and Mahanta and Das [85] explored the notions of fuzzy soft mappings. For the discussion on the algebraic structure binary lattice valued fuzzy relations, we refer to [102].

Investigation of fixed points in fuzzy soft topological spaces is an interesting area of research not yet explored. In this chapter, we initiate the study of fuzzy soft mappings and then obtain its fixed points. For this purpose we discuss some properties of a fuzzy soft element needed to prove our main result. A concept of fuzzy soft mapping is at the center of fixed point theory in the setup of fuzzy soft topology. We introduce fuzzy soft mappings with the help of Cartesian product and relations on fuzzy soft sets in fuzzy soft topological spaces. We prove that fuzzy soft continuous mappings preserve fuzzy soft compactness. This chapter also includes the fuzzy soft Cantor's intersection theorem. Finally we studied some necessary conditions for the existence of unique fuzzy soft element which serves as a fixed point of fuzzy soft mapping defined on a fuzzy soft compact Hausdorff topological space.

\subsection{Introduction and preliminaries}

Throughout this section, by $U, E$ and $P(U)$, we denote an initial universe, a set of parameters, and the collection of all subsets of $U$, respectively.

Definition 3.1.1 [136] A fuzzy set $A$ in $U$ is characterized by a function with domain as $U$ and values in $[0,1]$. The collection of all fuzzy sets in $U$ is 
denoted by $I^{U}$.

Definition 3.1.2 [136] An empty fuzzy set denoted by $\widetilde{0}$ is a function which maps each $x \in U$ to 0 . That is, $\widetilde{0}(x)=0$ for all $x \in U$. A universal fuzzy set denoted by $\widetilde{1}$ is a function which maps each $x \in U$ to 1 . That is, $\widetilde{1}(x)=1$ for all $x \in U$.

If $A, B \in I^{U}$ we write $A \preceq B$ whenever $A(x) \leq B(x)$ for each $x \in U$, and $A=B$ whenever $A \preceq B$ and $B \preceq A$ for all $x \in U$.

Definition 3.1.3 [136] Let $A$ and $B$ be two fuzzy sets. Then (a) their union $A \cup B$ is defined as $(A \cup B)(x)=\max \{A(x), B(x)\}$; (b) their intersection $A \cap B$ is defined as $(A \cap B)(x)=\min \{A(x), B(x)\}$, and (c) difference of $B$ from $A$ is denoted by $A / B$ and is defined by $(A / B)(x)=A(x)-B(x)$ for all $x \in U$.

Note that an implicit assumption $B \preceq A$ has been imposed to make the operation $A / B$ well defined.

Definition 3.1.4 [136] Then complement of a fuzzy set $A$ is denoted by $A^{c}$ and is defined by $A^{c}(x)=1-A(x)$.

Definition 3.1.5 [96] If $F$ is a mapping on $E$ taking values in $P(U)$, then a pair $(F, E)_{s}$ is called a soft set over $(U, E)$.

Definition 3.1.6 [86] Let $A$ be a subset of $E$. A pair $(F, A)$ is called a fuzzy soft set over $(U, E)$ if $F: A \rightarrow I^{U}$ is a mapping from $A$ into $I^{U}$. The collection of all fuzzy soft sets over $(U, E)$ is denoted by $\mathbf{F}(U, E)$.

A fuzzy soft set $(F, A)$ over $(U, E)$ is said to be:

(a) null fuzzy soft set if for each $e \in A, F(e)$ is a null fuzzy set $\widetilde{0}$ over $U$. We denote it by $\widetilde{\Phi}$.

(b) absolute fuzzy soft set if for each $e \in A, F(e)$ is a fuzzy universal set $\widetilde{1}$ over $U$. We denote it by $\widetilde{E}$.

Definition 3.1.7 [86] For two fuzzy soft sets $(F, A)$ and $(G, B)$ in $\mathbf{F}(U, E)$, we say that $(F, A) \widetilde{\subseteq}(G, B)$ if $A \subseteq B$ and $F(e) \preceq G(e)$ for each $e \in A$.

Definition 3.1.8 [86] Two fuzzy soft sets $(F, A)$ and $(G, B)$ in $\mathbf{F}(U, E)$ are equal if $F \widetilde{\subseteq} G$ and $G \widetilde{\subseteq} F$. 
Definition 3.1.9 [86] The difference between two fuzzy soft sets $(F, E),(G, E)$ in $\mathbf{F}(U, E)$ is a fuzzy soft set $(\widetilde{F / G}, E)$ (say) defined by $(\widetilde{F / G})(e)=F(e) / G(e)$ for each $e \in E$.

Definition 3.1.10 [86] The complement of a fuzzy soft set $(F, E)$ is a fuzzy soft set $\left(F^{\widetilde{c}}, E\right)$ defined by $F^{\widetilde{c}}(e)=\widetilde{1} / F(e)$ for each $e \in E$.

Clearly $F^{\widetilde{c}}=\widetilde{E} / F, \widetilde{\Phi}^{\widetilde{c}}=\widetilde{E}$, and $\left((F)^{\widetilde{c}}\right)^{\widetilde{c}}=F$.

Definition 3.1.11 $[6]$ Let $(F, A)$ and $(G, B)$ be two fuzzy soft sets in $\mathbf{F}(U, E)$ with $A \cap B \neq \widetilde{\Phi}$, then (d) their intersection $(F \widetilde{\cap} G, C)$ is a fuzzy soft set, where $C=A \cap B$ and, $(F \widetilde{\cap} G) e=F(e) \cap G(e)$ for each $e \in C$, and (e) their union $(F \widetilde{\cup} G, C)$ is a fuzzy soft set, where $\mathbf{C}=A \cup B$ and $(F \widetilde{\cup} G) e=F(e) \cup G(e)$ for each $e \in \mathbf{C}$.

Definition 3.1.12 [114] A fuzzy soft topology $\tau$ on $F \in \mathbf{F}(U, A)$ is a collection of fuzzy soft subsets of $F$ satisfying:

1. $\widetilde{\Phi}, F \in \tau$ ( this means that $\widetilde{E}$ is fuzzy soft subset of $F$, that is, $\widetilde{1}(e) \preceq F(e)$, that is $1 \leq F(e)(x)$

2. If $F_{1}, F_{2} \in \tau$ then $F_{1} \widetilde{\cap} F_{2} \in \tau$.

3. If $F_{\alpha} \in \tau$ for all $\alpha \in \Lambda$, with $\Lambda$ an index set, then $\widetilde{\cup}_{\alpha \in \Lambda} F_{\alpha} \in \tau$.

4. If $\tau$ is a fuzzy soft topology on $F$ then the pair $(F, \tau)$ is called a fuzzy soft topological space.

\subsection{Fuzzy soft elements}

Fuzzy soft element is defined as follows.

Definition 3.2.1 [100], [85] Let $e$ be any element in a set $A \subseteq E$. A fuzzy soft set $F$ over $A$ is called a fuzzy soft element if $F\left(e^{\prime}\right)$ is a null fuzzy set for each $e^{\prime} \in A-\{e\}$. We denote it by $\left(F^{e}, A\right)$ or simply by $F^{e}$

A fuzzy soft element $F^{e}$ is said to be in fuzzy soft set $(G, B)$ if $\left(F^{e}, A\right) \widetilde{\simeq}(G, B)$. That is, $A \subseteq B$ and $F^{e}\left(e^{\prime}\right) \preceq G\left(e^{\prime}\right)$ for each $e^{\prime} \in A$, that is, $F^{e}(e) \preceq G\left(e^{\prime}\right)$ for 
each $e^{\prime} \in A$. We write it as $F^{e} \tilde{\in} G$. It is straightforward to check that union of all fuzzy soft elements corresponding to each parameter $e \in A$ is equal to the approximate fuzzy soft set $F(e)$ and therefore the collection of all such unions, corresponding to each parameter, results in the original fuzzy soft set $(F, A)$.

Remark 3.2.2 Note that if $F$ is a fuzzy soft set in $\mathbf{F}(U, E)$ and $F^{e} \widetilde{\in} F$ then $F=\left\{\widetilde{\cup}_{F^{e} \tilde{\epsilon} F} F^{e}: e \in E\right\}$.

Example 3.2.3 Let $F$ be the fuzzy soft set in $\mathbf{F}(U, E)$ defined as

$$
F=\left\{\left(e_{1},\left\{\frac{u_{1}}{0.5}, \frac{u_{2}}{0.3}\right\}\right),\left(e_{2},\left\{\frac{u_{1}}{0.7}, \frac{u_{2}}{0.4}\right\}\right)\right\}
$$

Then some of the fuzzy soft elements of $F$ are

$$
\begin{aligned}
& F^{e_{1}}=\left\{\left(e_{1},\left\{\frac{u_{1}}{0.3}, \frac{u_{2}}{0.1}\right\}\right)\right\}, \digamma^{e_{1}}=\left\{\left(e_{1},\left\{\frac{u_{1}}{0.5}, \frac{u_{2}}{0.3}\right\}\right)\right\} \text { and } \\
& F^{e_{2}}=\left\{\left(e_{2},\left\{\frac{u_{1}}{0.7}, \frac{u_{2}}{0.4}\right\}\right)\right\} .
\end{aligned}
$$

Note that $F^{e_{1}} \widetilde{\cup} \digamma^{e_{1}}=\left\{\left(e_{1},\left\{\frac{u_{1}}{0.5}, \frac{u_{2}}{0.3}\right\}\right)\right\}=F\left(e_{1}\right)$. Similarly,

$$
\widetilde{\cup} F^{e_{2}}=\left\{\left(e_{2},\left\{\frac{u_{1}}{0.7}, \frac{u_{2}}{0.4}\right\}\right)=F\left(e_{2}\right)\right. \text {. }
$$

Therefore, $\left\{\widetilde{\cup}_{F^{e_{1} \in F}} F^{e_{1}}, \widetilde{\cup}_{F^{e_{2}}} F^{e_{2}}\right\}=F$.

Basic properties with held by fuzzy soft elements are stated in the following proposition.

Proposition 3.2.4 Let $F_{1}, F_{2}$ be two fuzzy soft sets over $(U, E)$ and $e \in E$ The following holds.

i) $\widetilde{\Phi}$ is an empty fuzzy soft element of every fuzzy soft set.

ii) If $F$ is a fuzzy soft set such that $F \neq \widetilde{\Phi}$, then $F$ contains at least one non empty fuzzy soft element.

iii) If $F^{e} \widetilde{\in} F_{1} \widetilde{\cup} F_{2}$ then $F^{e}$ is a fuzzy soft element of $F_{1}$ or $F_{2}$.

iv) $F^{e} \widetilde{\in} F_{1} \widetilde{\cap} F_{2}$ if and only if $F^{e}$ is a fuzzy soft element of $F_{1}$ and $F_{2}$. 
v) If $F^{e} \widetilde{\in} F_{1} \widetilde{\backslash} F_{2}$ then $F^{e}$ is a fuzzy soft element of $F_{1}$ but not necessarily a fuzzy soft element of $F_{2}$.

Proof. 1. Let $e$ be an element of $E$ and $F$ a fuzzy soft set over $E$. Obviously, $\widetilde{\Phi}(e) \preceq F(e)$ as $\widetilde{\Phi}(e)(x)=0$ for each $x \in U$. Therefore $\widetilde{\Phi}$ is an empty fuzzy soft element of every fuzzy soft set.

2. If $F \neq \widetilde{\Phi}$, then there exists at least one $e^{*} \in E$ such that $F\left(e^{*}\right) \neq \widetilde{0}$, that is, there exists an $x \in U$ for which $F\left(e^{*}\right)(x) \neq 0$. Let $F\left(e^{*}\right)(x)=\varepsilon$ for some $\varepsilon \in(0,1]$. Then we define $F_{1}$ such that

$$
F_{1}\left(e^{*}\right)(x)=\frac{\varepsilon}{2} \text { and } F_{1}(e)(x)=0 \text { whenever } e \neq e^{*} .
$$

This implies that $F_{1}\left(e^{*}\right) \preceq F\left(e^{*}\right)$. If $e \neq e^{*}$, then $\widetilde{0}=F_{1}(e) \preceq F(e)$. Hence fuzzy soft set $F_{1}$ is a non empty fuzzy soft element of $F$.

3. Let $F^{e}$ be a fuzzy soft element of $F_{1} \widetilde{\cup} F_{2}$, that is, $F^{e} \widetilde{\in}\left(F_{1} \widetilde{\cup} F_{2}\right)$ which implies that $F^{e}(e) \preceq F_{1}\left(e^{\prime}\right) \cup F_{2}\left(e^{\prime}\right)$ for each $e^{\prime} \in E$. So, for each $x \in U$, $F^{e}(e)(x) \leq \max \left\{F_{1}\left(e^{\prime}\right)(x), F_{2}\left(e^{\prime}\right)(x)\right\}$. Now if $F_{1}\left(e^{\prime}\right)(x) \leq F_{2}\left(e^{\prime}\right)(x)$ then for each $e^{\prime} \in E, F^{e}(e) \preceq F_{2}\left(e^{\prime}\right)$. Hence $F^{e} \widetilde{\in} F_{2}$. If $F_{2}\left(e^{\prime}\right)(x) \leq F_{1}\left(e^{\prime}\right)(x)$ then $F^{e}(e) \preceq F_{1}\left(e^{\prime}\right)$ for each $e^{\prime} \in E$ which implies that $F^{e} \widetilde{\in} F_{1}$. So, $F^{e} \widetilde{\in} F_{1}$ or $F^{e} \widetilde{\in} F_{2}$. Conversely, suppose that $F^{e} \widetilde{\in} F_{1}$ or $F^{e} \widetilde{\in} F_{2}$. Then $F^{e}(e) \preceq F_{1}\left(e^{\prime}\right)$ or $F^{e}(e) \preceq F_{2}\left(e^{\prime}\right)$ for each $e^{\prime} \in E$, that is, for all $x \in U, F^{e}(e)(x) \leq F_{1}\left(e^{\prime}\right)(x)$ or $F^{e}(e)(x) \leq F_{2}\left(e^{\prime}\right)(x)$. Thus $F^{e}(e)(x) \leq \max \left\{F_{1}\left(e^{\prime}\right)(x), F_{2}\left(e^{\prime}\right)(x)\right\}$. Therefore $F^{e} \widetilde{\in} F_{1} \widetilde{\cup} F_{2}$.

4. Let $F^{e} \widetilde{\in}\left(F_{1} \widetilde{\cap} F_{2}\right)$ which implies that $F^{e}(e) \preceq F_{1}\left(e^{\prime}\right) \cap F_{2}\left(e^{\prime}\right)$ for each $e^{\prime} \in E$. So for each $x \in U$,

$$
F^{e}(e)(x) \leq \min \left\{F_{1}\left(e^{\prime}\right)(x), F_{2}\left(e^{\prime}\right)(x)\right\}
$$

If $F_{1}\left(e^{\prime}\right)(x) \leq F_{2}\left(e^{\prime}\right)(x)$ then $F^{e}(e)(x) \leq F_{1}\left(e^{\prime}\right)(x) \leq F_{2}\left(e^{\prime}\right)(x)$ implies that $F^{e}$ is a fuzzy soft element of $F_{1}$ and $F_{2}$. Similarly if $F_{2}\left(e^{\prime}\right)(x) \leq F_{1}\left(e^{\prime}\right)(x)$ then $F^{e}(e)(x) \leq F_{2}\left(e^{\prime}\right)(x) \leq F_{1}\left(e^{\prime}\right)(x)$ means that $F^{e}$ is a fuzzy soft element 
of $F_{2}$ and $F_{1}$. Conversely, suppose that $F^{e} \tilde{\in} F_{1}$ and $F^{e} \widetilde{\in} F_{2}$. Then, for each $e^{\prime} \in E, F^{e}(e) \preceq F_{1}\left(e^{\prime}\right)$ and $F^{e}(e) \preceq F_{2}\left(e^{\prime}\right)$ which implies that

$$
F^{e}(e)(x) \leq \min \left\{F_{1}\left(e^{\prime}\right)(x), F_{2}\left(e^{\prime}\right)(x)\right\}
$$

for each $x$ in $U$. Therefore, $F^{e} \widetilde{\in} F_{1} \widetilde{\cap} F_{2}$.

5. Let $F^{e} \widetilde{\in} F_{1} \widetilde{\backslash} F_{2}$. Then, $F^{e}(e) \preceq F_{1}\left(e^{\prime}\right) \backslash F_{2}\left(e^{\prime}\right)$ for each $e^{\prime} \in E$, that is, $F^{e}(e)(x) \leq F_{1}\left(e^{\prime}\right)(x)-F_{2}\left(e^{\prime}\right)(x)$ for each $x \in U$. Then $F^{e}(e)(x) \leq F_{1}\left(e^{\prime}\right)(x)$ but the real number $F^{e}(e)(x)$ is not necessarily less than $F_{2}\left(e^{\prime}\right)(x)$ for each $x$. Therefore, $F^{e}$ is a fuzzy soft element of $F_{1}$ but $F^{e}$ is not necessarily a fuzzy soft element of $F_{2}$.

Example 3.2.5 Suppose that $U=\left\{u_{1}, u_{2}, u_{3}\right\}$ and $E=\left\{e_{1}, e_{2}\right\}$. Let $F, G \in \mathbf{F}(U, A)$ be of the form

$$
\begin{aligned}
F & =\left\{\left(e_{1},\left\{\frac{u_{1}}{0.6}, \frac{u_{2}}{0.8}, \frac{u_{3}}{0.3}\right\},\left(e_{2},\left\{\frac{u_{1}}{0.4}, \frac{u_{2}}{0.6}, \frac{u_{3}}{0.7}\right\}\right)\right\}\right. \text { and } \\
G & =\left\{\left(e_{1},\left\{\frac{u_{1}}{0.5}, \frac{u_{2}}{0.8}, \frac{u_{3}}{0.3}\right\},\left(e_{2},\left\{\frac{u_{1}}{0.2}, \frac{u_{2}}{0.4}, \frac{u_{3}}{0.3}\right\}\right)\right\} .\right.
\end{aligned}
$$

Note that

$$
\begin{aligned}
& F \widetilde{\cup} G=\left\{\left(e_{1},\left\{\frac{u_{1}}{0.6}, \frac{u_{2}}{0.8}, \frac{u_{3}}{0.3}\right\},\left(e_{2},\left\{\frac{u_{1}}{0.4}, \frac{u_{2}}{0.6}, \frac{u_{3}}{0.7}\right\}\right)\right\},\right. \\
& F \widetilde{\cap} G=\left\{\left(e_{1},\left\{\frac{u_{1}}{0.5}, \frac{u_{2}}{0.8}, \frac{u_{3}}{0.3}\right\},\left(e_{2},\left\{\frac{u_{1}}{0.2}, \frac{u_{2}}{0.4}, \frac{u_{3}}{0.3}\right\}\right)\right\},\right. \text { and } \\
& F \widetilde{\nearrow G}=\left\{\left(e_{1},\left\{\frac{u_{1}}{0.1}\right\},\left(e_{2},\left\{\frac{u_{1}}{0.2}, \frac{u_{2}}{0.2}, \frac{u_{3}}{0.4}\right\}\right)\right\} .\right.
\end{aligned}
$$

$F^{e_{1}}=\left\{\left(e_{1},\left\{\frac{u_{1}}{0.4}, \frac{u_{2}}{0.1}, \frac{u_{3}}{0.3}\right\}\right)\right\}$ is a soft fuzzy element of $F$. Note that $F^{e_{1}} \widetilde{\in} F \widetilde{\cup} G$. Similarly, $F^{e_{1}} \widetilde{\in} F \widetilde{\cap} G$. Also, $F^{e_{2}}=\left\{\left(e_{2},\left\{\frac{u_{1}}{0.1}, \frac{u_{2}}{0.1}, \frac{u_{3}}{0.4}\right\}\right)\right\}$ is a soft fuzzy point of $F \widetilde{\backslash G}$ then $F^{e_{2}} \widetilde{\in} F$ but $F^{e_{2}}$ is not a fuzzy soft element of $G$.

Proposition 3.2.6 Let $F_{1}, F_{2}$ be two fuzzy soft sets over $E$. Then $F_{1} \widetilde{\subseteq} F_{2}$ if and only if $F^{e} \widetilde{\in} F_{1}$ implies that $F^{e} \widetilde{\in} F_{2}$.

Proof. Let $F_{1} \widetilde{\subseteq} F_{2}$ then $F_{1}(e) \preceq F_{2}(e)$ for each $e \in E$, that is $F_{1}(e)(x) \leq$ $F_{2}(e)(x)$ for each $x \in U$. Suppose that $F^{e} \tilde{\in} F_{1}$. That is, for each $e^{\prime} \in E$, $F^{e}(e) \preceq F_{1}\left(e^{\prime}\right)$ and hence $F^{e}(e) \preceq F_{2}\left(e^{\prime}\right)$ for each $e^{\prime} \in E$. Therefore, $F^{e} \widetilde{\in} F_{2}$. 
Conversely, suppose that every fuzzy soft element $F^{e}$ in $F_{1}$ is also a fuzzy soft element of $F_{2}$. Let $\bar{F}_{1}^{e}$ to be the largest fuzzy soft element of $F_{1}$ for each $e \in E$ then $\bar{F}_{1}^{e} \widetilde{\in} F_{2}$. Let $\varepsilon \in(0,1]$ and $\bar{F}_{1}^{e}(e)(x)+\varepsilon$ be such that $\bar{F}_{1}^{e}(e)(x)+\varepsilon \leq$ $F_{2}\left(e^{\prime}\right)(x)$ for each $x \in U$. That is, $\bar{F}_{1}^{e}(e)(x) \leq F_{2}\left(e^{\prime}\right)(x)$ for each $e^{\prime} \in E$. Therefore, $F_{1} \simeq F_{2}$.

Definition 3.2.7 [85] A fuzzy soft topological space $(F, \tau)$ is said to be a fuzzy soft Hausdorff space if for distinct fuzzy soft elements $F^{e}, F^{e^{\prime}}$ of $F$, there exists disjoint fuzzy soft open sets $\left(F_{1}, A\right)$ and $\left(F_{2}, A\right)$ such that $F^{e} \tilde{\in} F_{1}$ and $F^{e^{\prime}} \tilde{\in} F_{2}$.

Proposition 3.2.8 Let $(F, \tau)$ be a fuzzy soft topological space. A fuzzy soft set $V \widetilde{\subseteq} F$ is fuzzy soft open if and only if for each $F^{e} \widetilde{\in} V$ there exists a fuzzy soft set $W \widetilde{\in} \tau$ such that $F^{e} \widetilde{\in} W \widetilde{\subseteq} V$.

Proof. Let $V \in \tau$. Then clearly for each $F^{e} \widetilde{\in} V$ we have $F^{e} \widetilde{\in} V \widetilde{\widetilde{c}} V$. Let $V \widetilde{\subseteq} F$ be such that for each $F^{e} \widetilde{\in} V$ there exists a fuzzy soft open set $W_{F^{e}}$ such that $F^{e} \widetilde{\in} W_{F^{e}} \widetilde{\widetilde{C}} V$ which means that $F^{e}(e) \preceq W_{F^{e}}\left(e^{\prime}\right) \preceq V\left(e^{\prime}\right)$ for each $e^{\prime} \in E$. By Remark 3.2.2, for each $e \in E, V(e)=\widetilde{\cup}\left\{F^{e}: F^{e} \widetilde{\in} V\right\} \widetilde{\subseteq} \widetilde{\cup} W_{F^{e}}(e) \widetilde{\widetilde{\subseteq}} V(e)$. Therefore, $V=\left\{\widetilde{U} W_{F^{e}}: e \in E\right\} \in \tau$.

\subsection{Fuzzy soft mappings}

In this section, a concept of fuzzy soft mapping is introduced. Relevant definitions are formulated and some properties of fuzzy soft mappings are studied.

Definition 3.3.1 [10] The Cartesian product of two fuzzy soft sets $(F, A)$ and $(G, B)$ is defined as a fuzzy soft set $(H, C)=(F, A) \widehat{\times}(G, B)$, where $C=A \times B$ and $H: C \rightarrow \mathbf{F}(U, E)$ is defined by

$$
H\left(e, e^{\prime}\right)=F(e) \widetilde{\times} G\left(e^{\prime}\right)
$$

for all $\left(e, e^{\prime}\right) \in C$, where $F(e) \widetilde{\times} G\left(e^{\prime}\right)=\left\{\frac{x}{\min \left\{F\left(e^{\prime}\right)(x), G\left(e^{\prime}\right)(x)\right.}: x \in U\right\}$. 
Example 3.3.2 Let $U=\left\{u_{1}, u_{2}\right\}$ and $A=\left\{e_{1}, e_{2}, e_{3}\right\}$. Define fuzzy soft sets $F_{1}$ and $F_{2}$ as follows:

$$
\begin{aligned}
& \left(F_{1}, A\right)=\left\{\left(e_{1},\left\{\frac{u_{1}}{0.6}, \frac{u_{2}}{0.5}\right\}\right),\left(e_{2},\left\{\frac{u_{1}}{0.3}, \frac{u_{2}}{0.5}\right\}\right),\left(e_{3},\left\{\frac{u_{1}}{0.2}, \frac{u_{2}}{0.7}\right\}\right)\right\} \text { and } \\
& \left(F_{2}, A\right)=\left\{\left(e_{1},\left\{\frac{u_{1}}{0.3}, \frac{u_{2}}{0.4}\right\}\right),\left(e_{2},\left\{\frac{u_{1}}{0.6}, \frac{u_{2}}{0.7}\right\}\right),\left(e_{3},\left\{\frac{u_{1}}{0.5}, \frac{u_{2}}{0.4}\right\}\right)\right\} .
\end{aligned}
$$

Then $\left(F_{1}, A\right) \widehat{\times}\left(F_{2}, A\right)=(H, C)$ where $C=A \times A$ and $H$ is given by

$$
\begin{aligned}
& H\left(e_{1}, e_{1}\right)=F_{1}\left(e_{1}\right) \widetilde{\times} F_{2}\left(e_{1}\right)=\left\{\frac{u_{1}}{0.3}, \frac{u_{2}}{0.4}\right\}, \\
& H\left(e_{1}, e_{2}\right)=F_{1}\left(e_{1}\right) \widetilde{\times} F_{2}\left(e_{2}\right)=\left\{\frac{u_{1}}{0.6}, \frac{u_{2}}{0.5}\right\}, \\
& H\left(e_{1}, e_{3}\right)=F_{1}\left(e_{1}\right) \widetilde{\times} F_{2}\left(e_{3}\right)=\left\{\frac{u_{1}}{0.5}, \frac{u_{2}}{0.4}\right\}, \\
& H\left(e_{2}, e_{1}\right)=F_{1}\left(e_{2}\right) \widetilde{\times} F_{2}\left(e_{1}\right)=\left\{\frac{u_{1}}{0.3}, \frac{u_{2}}{0.4}\right\}, \\
& H\left(e_{2}, e_{2}\right)=F_{1}\left(e_{2}\right) \widetilde{\times} F_{2}\left(e_{2}\right)=\left\{\frac{u_{1}}{0.3}, \frac{u_{2}}{0.5}\right\}, \\
& H\left(e_{2}, e_{3}\right)=F_{1}\left(e_{2}\right) \widetilde{\times} F_{2}\left(e_{3}\right)=\left\{\frac{u_{1}}{0.3}, \frac{0.4}{u_{2}}\right\}, \\
& H\left(e_{3}, e_{1}\right)=F_{1}\left(e_{1}\right) \widetilde{\times} F_{2}\left(e_{1}\right)=\left\{\frac{u_{1}}{0.2}, \frac{u_{2}}{0.4}\right\}, \\
& H\left(e_{3}, e_{2}\right)=F_{1}\left(e_{1}\right) \widetilde{\times} F_{2}\left(e_{1}\right)=\left\{\frac{u_{1}}{0.2}, \frac{u_{2}}{0.7}\right\}, \\
& H\left(e_{3}, e_{3}\right)=F_{1}\left(e_{1}\right) \widetilde{\times} F_{2}\left(e_{1}\right)=\left\{\frac{u_{1}}{0.2}, \frac{u_{2}}{0.4}\right\} .
\end{aligned}
$$

Definition 3.3.3 Let $\left(F_{1}, A\right),\left(F_{2}, A\right)$ be fuzzy soft sets in $\mathbf{F}(U, E)$. A fuzzy soft set $R$ is called a fuzzy soft relation from $F_{1}$ to $F_{2}$ if $R=(G, D)$ where $D \subseteq C$ and $G=H$ on $D$.

Example 3.3.4 Let $F_{1}, F_{2}$ be as given in Example 3.3.2. Then

$$
R=\left\{F_{1}\left(e_{1}\right) \widetilde{\times} F_{2}\left(e_{2}\right), F_{1}\left(e_{2}\right) \widetilde{\times} F_{2}\left(e_{3}\right), F_{1}\left(e_{3}\right) \widetilde{\times} F_{2}\left(e_{3}\right)\right\}
$$

is a fuzzy soft relation from $F_{1}$ to $F_{2}$ which itself is a fuzzy soft set with $\left\{\left(e_{1}, e_{1}\right),\left(e_{2}, e_{3}\right),\left(e_{3}, e_{3}\right)\right\}$ as a set of parameters. By $F_{1} R F_{2}$, we mean that $F_{1}\left(e_{1}\right) \widetilde{\times} F_{2}\left(e_{2}\right) \in R$. 
We now introduce a fuzzy soft mapping.

Definition 3.3.5 Let $F, G$ be fuzzy soft sets in $\mathbf{F}(U, E)$. A fuzzy soft relation $T$ from $F$ to $G$ is called a fuzzy soft mapping from $F$ to $G$ denoted by $T: F \rightarrow G$ if the following conditions are satisfied.

C1 for each fuzzy soft element $F^{e} \widetilde{\in} F$, there exists only one fuzzy soft element $G^{e} \widetilde{\in} G$ such that $F^{e} T G^{e}$ which will be denoted as $T\left(F^{e}\right)=G^{e}$.

C2 for each fuzzy soft empty element $F^{e} \widetilde{\in} F, T\left(F^{e}\right)$ is a empty fuzzy soft element of $G$.

Definition 3.3.6 Let $F, G$ be fuzzy soft sets in $\mathbf{F}(U, E)$ and $T: F \rightarrow G$ a fuzzy soft mapping. The image of $X \widetilde{\widetilde{C}} F$ under soft fuzzy mapping $T$ is the fuzzy soft set $T(X)$ defined by

$$
T(X)=\left\{\widetilde{\cup}_{F^{e} \tilde{\in} X} T\left(F^{e}\right): e \in E\right\} .
$$

It is clear that $T(\widetilde{\Phi})=\widetilde{\Phi}$ for each fuzzy soft mapping $T$.

Definition 3.3.7 Let $F, G \in \mathbf{F}(U, A)$ and $T: F \rightarrow G$ a soft fuzzy mapping. The inverse image of $Y \widetilde{\subseteq} G$ under fuzzy soft mapping $T$ is the fuzzy soft set denoted by $T^{-1}(Y)$ and defined as:

$$
T^{-1}(Y)=\left\{\left\{\widetilde{\cup}_{F^{e}} \tilde{\epsilon}_{F} F^{e}: e \in E\right\}: T\left(F^{e}\right) \widetilde{\in} Y \text { for each } e \in E\right\}
$$

Example 3.3.8 Let $F$ and $G$ be defined as:

$$
\begin{aligned}
& F=\left\{\left(e_{1},\left\{\frac{u_{1}}{0.6}, \frac{u_{2}}{0.4}\right\}\right),\left(e_{2},\left\{\frac{u_{1}}{0.3}, \frac{u_{2}}{0.7}\right\}\right)\right\} \text { and } \\
& G=\left\{\left(e_{1},\left\{\frac{u_{1}}{0.2}, \frac{u_{2}}{0.6}\right\}\right),\left(e_{2},\left\{\frac{u_{1}}{0.7}, \frac{u_{2}}{0.8}\right\}\right)\right\} .
\end{aligned}
$$

Define $T$ as $T\left(F^{e}\right)=\widehat{G^{e}}$ for each $e \in E$, where $\widehat{G^{e}}$ is the largest fuzzy soft element corresponding to each parameter $e \in E$, that is, if $G^{e}$ is any fuzzy soft element in $G$ then $G^{e} \widetilde{\subseteq} \widehat{G^{e}}$. So, $T\left(F^{e_{1}}\right)=\widehat{G^{e_{1}}}=\left\{\frac{u_{1}}{0.2}, \frac{u_{2}}{0.6}\right\}$ for all $F^{e_{1}} \widetilde{\in} F$ 
and $T\left(F^{e_{2}}\right)=\widehat{G^{e_{2}}}=\left\{\frac{u_{1}}{0.7}, \frac{u_{2}}{0.8}\right\}$ for all $F^{e_{2}} \widetilde{\in} F$. Moreover,

$$
\begin{aligned}
T(F) & =\left\{\cup_{F^{e} \widetilde{\in} X} T\left(F^{e}\right): e \in E\right\}=\left\{\left\{\cup_{F^{e_{1}} \tilde{\in} X} T\left(F^{e_{1}}\right)\right\},\left\{\cup_{F^{e_{2}} \tilde{\in} X} T\left(F^{e_{2}}\right)\right\}\right\} \\
& =\left\{\widehat{G^{e_{1}}}, \widehat{G^{e_{2}}}\right\}=G .
\end{aligned}
$$

Proposition 3.3.9 Let $F, G \in \mathbf{F}(\mathrm{U}, \mathrm{E}),(\mathrm{X}, \mathrm{E}),\left(\mathrm{X}_{1}, E\right),\left(X_{2}, E\right) \widetilde{\subseteq}(F, E)$, and $(Y, E),\left(Y_{1}, E\right),\left(Y_{2}, E\right) \widetilde{\subseteq}(G, E)$. Let $T: F \rightarrow G$ be a fuzzy soft mapping. Then following hold.

i. $X_{1} \widetilde{\widetilde{C}} X_{2} \Rightarrow T\left(X_{1}\right) \widetilde{\simeq} T\left(X_{2}\right)$,

ii. $Y_{1} \widetilde{\widetilde{C}} Y_{2} \Rightarrow T^{-1}\left(Y_{1}\right) \widetilde{\widetilde{C}} T^{-1}\left(Y_{2}\right)$,

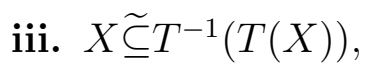

iv. $T\left(T^{-1}(Y)\right) \widetilde{\subseteq} Y$,

v. $T\left(X_{1} \widetilde{\cup} X_{2}\right)=T\left(X_{1}\right) \widetilde{\cup} T\left(X_{2}\right)$,

vi. $T\left(X_{1} \widetilde{\cap} X_{2}\right)=T\left(X_{1}\right) \widetilde{\cap} T\left(X_{2}\right)$,

vii. $T^{-1}\left(Y_{1} \widetilde{\cup} Y_{2}\right)=T^{-1}\left(Y_{1}\right) \tilde{\cup} T^{-1}\left(Y_{2}\right)$, and

Viii $T^{-1}\left(Y_{1} \widetilde{\cap} Y_{2}\right)=T^{-1}\left(Y_{1}\right) \widetilde{\cap} T^{-1}\left(Y_{2}\right)$.

Proof. i. Let $F^{e}$ be an arbitrary fuzzy soft element in $T\left(X_{1}\right)$ then there exists a fuzzy soft element $\digamma^{e}$ in $X_{1}$ such that $T\left(\digamma^{e}\right)=F^{e}$. As $X_{1} \widetilde{\simeq} X_{2}$ so $\digamma^{e}$ is a fuzzy soft element of $X_{2}$. So for every fuzzy soft element $F^{e}$ in $T\left(X_{1}\right)$, $F^{e}$ is a fuzzy soft element in $T\left(X_{2}\right)$. Hence the result.

v. Let $\digamma^{e} \widetilde{\in} T\left(X_{1} \widetilde{\cup} X_{2}\right)$. Then $\digamma^{e}=T\left(F^{e}\right)$ for some $F^{e} \widetilde{\in} X_{1} \widetilde{\cup} X_{2}$. If $F^{e} \widetilde{\in} X_{1}$ then $\digamma^{e} \widetilde{\in} T\left(X_{1}\right) \widetilde{\subseteq} T\left(X_{1}\right) \widetilde{\cup} T\left(X_{2}\right)$ and if $F^{e} \widetilde{\in} X_{2}$ then $\digamma^{e} \widetilde{\in} T\left(X_{2}\right) \widetilde{\subseteq} T\left(X_{1}\right) \tilde{\cup} T\left(X_{2}\right)$. Therefore, $T\left(X_{1} \widetilde{\cup} X_{2}\right) \widetilde{\subseteq} T\left(X_{1}\right) \widetilde{\cup} T\left(X_{2}\right)$. Now let $\digamma^{e} \widetilde{\in} T\left(X_{1}\right) \widetilde{\cup} T\left(X_{2}\right)$, that is, $\digamma^{e}$ is fuzzy soft element of $T\left(X_{1}\right)$ or $T\left(X_{2}\right)$. If $F^{e} \widetilde{\in} T\left(X_{1}\right)$, then $T\left(X_{1}\right) \widetilde{\subseteq} T\left(X_{1} \widetilde{\cup} X_{2}\right)$ 
gives $F^{e} \tilde{\in} T\left(X_{1} \widetilde{\cup} X_{2}\right)$. Similarly, If $F^{e} \tilde{\in} T\left(X_{2}\right)$, then $T\left(X_{2}\right) \widetilde{\subseteq} T\left(X_{1} \widetilde{\cup} X_{2}\right)$ gives $F^{e} \tilde{\in} T\left(X_{1} \widetilde{\cup} X_{2}\right)$. Therefore $T\left(X_{1}\right) \widetilde{\cup} T\left(X_{2}\right) \widetilde{\subseteq} T\left(X_{1} \widetilde{\cup} X_{2}\right)$. So we conclude that

$$
T\left(X_{1} \widetilde{\cup} X_{2}\right)=T\left(X_{1}\right) \widetilde{\cup} T\left(X_{2}\right) .
$$

viii. If $F^{e} \widetilde{\in} T^{-1}\left(Y_{1} \widetilde{\cap} Y_{2}\right)$ then $T\left(F^{e}\right) \widetilde{\in} Y_{1} \widetilde{\cap} Y_{2}$. Since for each $e \in E$, $T\left(F^{e}\right) \widetilde{\simeq} Y_{1}(e) \cap Y_{2}(e)$, then, for all $x, T\left(F^{e}\right)(x)$ is less than the minimum of $Y_{1}(e)(x)$ and $Y_{2}(e)(x)$. Hence, $F^{e} \widetilde{\in} T^{-1}\left(Y_{1}\right) \widetilde{\cap} T^{-1}\left(Y_{2}\right)$ and therefore,

$$
T^{-1}\left(Y_{1} \widetilde{\cap} Y_{2}\right) \widetilde{\simeq} T^{-1}\left(Y_{1}\right) \widetilde{\cap} T^{-1}\left(Y_{2}\right)
$$

Now, let $F^{e} \tilde{\in} T^{-1}\left(Y_{1}\right) \widetilde{\cap} T^{-1}\left(Y_{2}\right)$. Then following similar arguments to those given above it follows that $T\left(F^{e}\right) \widetilde{\in} Y_{1}$ and $T\left(F^{e}\right) \widetilde{\in} Y_{2}$. It follows from here that $F^{e} \tilde{\in} T^{-1}\left(Y_{1} \widetilde{\cap} Y_{2}\right)$. So, $T^{-1}\left(Y_{1}\right) \widetilde{\cap} T^{-1}\left(Y_{2}\right) \widetilde{\subseteq} T^{-1}\left(Y_{1} \widetilde{\cap} Y_{2}\right)$.

Proofs of the rest of the properties follow on similar lines.

Definition 3.3.10 Let $(F, \tau)$ be a fuzzy soft topological space and $K \widetilde{\subseteq} F$. A fuzzy soft open cover for $K$ is a collection of fuzzy soft open sets $\left\{V_{i}\right\}_{i \in I} \subseteq \tau$ whose union contains $K$.

Definition 3.3.11 A fuzzy soft topological space $(F, \tau)$ is compact if for each fuzzy soft open cover $\left\{V_{i}\right\}_{i \in I}$ of $K$ there exists $i_{1}, i_{2}, . ., i_{k} \in I, k \in \mathbb{N}$ such that $K \widetilde{\subseteq} \cup_{n=1}^{k} V_{i_{n}}$.

Definition 3.3.12 Let $(F, \tau),(G, v)$ be fuzzy soft topological spaces and $T: F \rightarrow G$ a soft fuzzy mapping. Then $T$ is a fuzzy soft continuous mapping (with respect to the fuzzy soft topologies $\tau$ and $v$ ) if for each $V \in v, T^{-1}(V) \in$ $\tau$, that is, the inverse image of a fuzzy soft open set is a fuzzy soft open set.

We say that the fuzzy soft set $K \widetilde{\subseteq} F$ is fuzzy soft compact in $(F, \tau)$ if the fuzzy soft topological space $\left(K, \tau_{\mid K}\right)$ is fuzzy soft compact.

Example 3.3.13 Let $U=\left\{u_{1}, u_{2}, u_{3}\right\}, E=\left\{e_{1}, e_{2}, e_{3}\right\}$. Suppose $F \in$ $\mathbf{F}(U, A)$ is of the form $F=\left\{\left(e_{1},\left\{\frac{u_{1}}{1}, \frac{u_{2}}{1}, \frac{u_{3}}{0.7}\right\}\right),\left(e_{2},\left\{\frac{u_{1}}{0.6}, \frac{u_{2}}{0.9}, \frac{u_{3}}{0.7}\right\}\right)\right\}$. Consider the family $\tau$ of all fuzzy soft subsets of $F$ and let $V=\widehat{F^{e_{1}}} \in \tau$ where $\widehat{F^{e}}$ is the largest fuzzy soft element of $F$. Define $T: F \rightarrow F$ as $T\left(F^{e}\right)=F^{e}$ for each $e \in E$. Then, $T^{-1}\left(\widehat{F^{e_{1}}}\right)=\widehat{F^{e_{1}}} \in \tau$. 
Proposition 3.3.14 Let $(K, \tau)$ be a fuzzy soft compact topological space and $T: K \rightarrow K$ a fuzzy soft continuous mapping. Then $T(K)$ is a fuzzy soft compact set in $(K, \tau)$.

Proof. Suppose that $T(K) \widetilde{\widetilde{\subseteq}} \widetilde{\cup}_{\ell} G_{\ell}$, where $\left\{G_{\ell}\right\}$ is a family of fuzzy soft open sets in $K$. Then taking the preimage, we have, $K \widetilde{\widetilde{C}} T^{-1}\left(\widetilde{\cup}_{\ell} G_{\ell}\right)$. As $T^{-1}\left(G_{\ell}\right)$ is open in $K$ so there must exist soft fuzzy open $V_{\ell} \widetilde{\subseteq} T(K)$ such that $T^{-1}\left(G_{\ell}\right)$ $=V_{\ell} \widetilde{\cap} K$. So $K \widetilde{\subseteq} \widetilde{\cup}_{\ell}\left(V_{\ell} \widetilde{\cap} K\right)$ implies that $K \widetilde{\subseteq} \widetilde{\cup}_{\ell} V_{\ell}$. Since $K$ is compact fuzzy soft set, therefore there exist $\ell_{1}, \ell_{2}, \ldots, \ell_{N}$ such that $K \widetilde{\subseteq} \widetilde{\cup}_{i=1}^{N} V_{\ell_{i}}$. Hence $K=\widetilde{\cup}_{\ell}\left(V_{\ell} \widetilde{\cap} K\right)=\widetilde{\cup}_{i=1}^{N} T^{-1}\left(G_{\ell_{i}}\right)$ which implies that $T(K) \widetilde{\widetilde{\cup}}_{i=1}^{N} G_{\ell_{i}}$. Hence $T(K)$ is compact.

\subsection{Fixed points of fuzzy soft mappings}

We start this section with the definition of a fixed point of a fuzzy soft mapping.

Definition 3.4.1 Let $F \in \mathbf{F}(U, A)$ be a fuzzy soft set and $T: F \rightarrow F$ a fuzzy soft mapping. A fuzzy soft element $F^{e} \widetilde{\in} F$ is called a fixed point of $T$ if $T\left(F^{e}\right)=F^{e}$.

Example 3.4.2 If $T: F \rightarrow F$ is defined as an identity map, then each fuzzy soft element of $F$ is a fixed point.

Proposition 3.4.3 Let $(F, \tau)$ be a fuzzy soft compact topological space and $\left\{F_{n}: n \in \mathbb{N}\right\}$ a countable family of fuzzy soft subsets of $F$ satisfying:

AI. $F_{n} \neq \widetilde{\Phi}$ for each $n \in \mathbb{N}$,

A2. $F_{n}$ is fuzzy soft closed for each $n \in \mathbb{N}$,

A3. $F_{n+1} \widetilde{\widetilde{C}} F_{n}$ for each $n \in \mathbb{N}$.

Then $\widetilde{\cap}_{n \in \mathbb{N}} F_{n} \neq \widetilde{\Phi}$.

Proof. Suppose on the contrary, that $\widetilde{\cap}_{n \in \mathbb{N}} F_{n}=\widetilde{\Phi}$. We know that $\left(\widetilde{\cap}_{n \in \mathbb{N}} F_{n}\right)^{\widetilde{c}}=\widetilde{\cup}_{n \in \mathbb{N}}\left(F_{n}\right)^{\widetilde{c}}$ (see $\left.[6]\right)$. From $(A 2),\left(F_{n}\right)^{\widetilde{c}}$ is a fuzzy soft open 
set for each $n \in \mathbb{N}$. Hence

$$
F \widetilde{\widetilde{E}} \widetilde{E}=(\widetilde{\Phi})^{\widetilde{c}}=\left(\widetilde{\cap}_{n \in \mathbb{N}} F_{n}\right)^{\widetilde{c}}=\widetilde{\cup}_{n \in \mathbb{N}}\left(F_{n}\right)^{\widetilde{c}}
$$

As $F$ is fuzzy soft compact, there exists $i_{1}, i_{2}, \ldots, i_{k} \in \mathbb{N}, i_{1}<i_{2}<\ldots<i_{k}$, $k \in \mathbb{N}$ such that

$$
F \widetilde{\subseteq} F_{i_{1}}^{\widetilde{u}} \widetilde{\cup} F_{i_{2}}^{\widetilde{c}}, \ldots \widetilde{\cup} F_{i_{k}}^{\widetilde{c}}
$$

Hence from $(A 3)$, we have, $F_{i_{k}} \widetilde{\widetilde{C}} F \widetilde{\subseteq}\left(F_{i_{1}} \widetilde{\cap} F_{i_{2}} \widetilde{\cap} \ldots \widetilde{\cap} F_{i_{k}}\right)^{\widetilde{c}}=F_{i_{k}}^{\widetilde{c}}=\widetilde{E} / F_{i_{k}}$, which is impossible in the light of $(A 1)$.

Example 3.4.4 Let $(F, \tau)$ be a fuzzy soft topological space where $\tau$ contains all possible subsets of $F=\left\{\left(e_{1},\left\{\frac{u_{1}}{1}, \frac{u_{2}}{0.7}\right\}\right),\left(e_{2},\left\{\frac{u_{1}}{0.9}, \frac{u_{2}}{1}\right\}\right)\right\}$. Let two fuzzy soft subsets of $F$ be defined as

$$
F_{1}=\left\{\left(e_{1},\left\{\frac{u_{1}}{0.4}, \frac{u_{2}}{0.5}\right\}\right),\left(e_{2},\left\{\frac{u_{1}}{0.8}, \frac{u_{2}}{0.4}\right\}\right)\right\}
$$

and

$$
F_{2}=\left\{\left(e_{1},\left\{\frac{u_{1}}{0.6}, \frac{u_{2}}{0.3}\right\}\right),\left(e_{2},\left\{\frac{u_{1}}{0.8}, \frac{u_{2}}{0.5}\right\}\right)\right\} .
$$

Note that they satisfy the conditions of proposition 36 . Moreover $F_{1} \widetilde{\subseteq} F_{2}$ and $\widetilde{\cap}_{j=1}^{2} F_{j}=F_{1} \neq \widetilde{\Phi}$.

Proposition 3.4.5 Let $(F, \tau)$ be a fuzzy soft topological space and $T$ : $F \rightarrow F$ a fuzzy soft mapping such that for each nonempty fuzzy soft element $F^{e} \widetilde{\in} F, T\left(F^{e}\right)$ is a non empty fuzzy soft element of $F$. If $\widetilde{\cap}_{n \in \mathbb{N}} T^{n}(F)$ contains only one nonempty fuzzy soft element $F^{e} \widetilde{\in} F$, then $F^{e}$ is a unique fixed point of $T$.

Proof. Observe that $T^{n}(F) \widetilde{\widetilde{\subseteq}} T^{n-1}(F)$ for each $n \in \mathbb{N}$. Let $F^{e}$ be a fuzzy soft element of $F$ such that $F^{e} \widetilde{\in} \widetilde{\cap}_{n \in \mathbb{N}} T^{n}(F)$. That is, $F^{e} \widetilde{\widetilde{\cap}} \widetilde{\cap}_{n \in \mathbb{N}} T^{n}(F)$. Consequently

$$
T\left(F^{e}\right) \widetilde{\widetilde{\subseteq}} T\left(\widetilde{\cap}_{n \in \mathbb{N}} T^{n}(F)\right) \widetilde{\widetilde{\subseteq}} \widetilde{\cap}_{n \in \mathbb{N}} T^{n+1}(F) \widetilde{\widetilde{\subseteq}} \widetilde{\cap}_{n \in \mathbb{N}} T^{n}(F)=F^{e}
$$

Since $T\left(F^{e}\right)$ is a non empty fuzzy soft element of $F$, therefore we obtain that $T\left(F^{e}\right)=F^{e}$. 
Example 3.4.6 Let $(F, \tau)$ be a fuzzy soft topological space and define $T: F \rightarrow F$ as $T\left(F^{e}\right)=\widehat{F^{e}}$ for all $F^{e} \widetilde{\in} F$, where $F \neq \widetilde{\Phi}$ and $\widehat{F^{e}}$ represents the largest fuzzy soft element of $F$ or equivalently $F^{e} \widetilde{\subseteq} \widehat{F^{e}}$ for each fuzzy soft element $F^{e} \widetilde{\in} F$. Then $\widetilde{\cap}_{n \in \mathbb{N}} T^{n}(F)$ contains only one non empty fuzzy soft element which is $\widehat{F e}$. Note that $\widehat{F^{e}}$ is a unique fixed point of $T$.

Proposition 3.4.7 Let $(F, \tau)$ be a fuzzy soft Hausdorff topological space. Then every fuzzy soft compact set in $F$ is fuzzy soft closed in $F$.

Proof. Let $K$ be a fuzzy soft compact set in $(F, \tau)$. We need to show that $K$ is fuzzy soft closed, that is, $K^{\widetilde{c}}$ is fuzzy soft open. Let $F^{e} \widetilde{\in} K^{\widetilde{c}}$. For every $F^{e^{\prime}} \tilde{\in} K$, let $U_{i}, V_{i} \in \tau$ be such that $U_{i} \widetilde{\cap} V_{i}=\widetilde{\Phi}$ and $F^{e} \widetilde{\in} U_{i}, F^{e^{\prime}} \widetilde{\in} V_{i}$ where $i \in I$. Since $K$ is fuzzy soft compact so there exists $F^{e^{\prime}}, F^{e^{\prime}}, \ldots, F^{e^{\prime}} \widetilde{\in} K$ such that $K \widetilde{\subseteq} V_{i_{1}} \widetilde{\cup} V_{i_{2}} \widetilde{\cup} \ldots \widetilde{\cup} V_{i_{k}}$. Denote $U=U_{i_{1}} \widetilde{\cup} U_{i_{2}} \widetilde{\cup} \ldots \widetilde{\cup} U_{i_{k}}$ and $V=V_{i_{1}} \widetilde{\cup} V_{i_{2}} \widetilde{\cup} \ldots \widetilde{\cup} V_{i_{k}}$. Then $F^{e} \widetilde{\in} U \in \tau, U \widetilde{\cap} V=\widetilde{\Phi}$, which gives that $F^{e} \widetilde{\in} U \widetilde{\subseteq} K^{\widetilde{c}}$. Therefore $K$ is fuzzy soft closed.

Theorem 3.4.8 Let $(K, \tau)$ be a fuzzy soft compact Hausdorff topological space and $T: K \rightarrow K$ a fuzzy soft continuous mapping such that

(a). for each non empty fuzzy soft element $F^{e} \widetilde{\in} K, T\left(F^{e}\right)$ is a non empty fuzzy soft element of $K$,

(b). for each fuzzy soft closed set $X \widetilde{\widetilde{C}} K$ if $T(X)=X$ then $X$ contains only one non empty fuzzy soft element of $K$.

Then there exists a unique nonempty fuzzy soft element $F^{e} \widetilde{\in} K$ such that $T\left(F^{e}\right)=F^{e}$.

Proof. Consider a family of fuzzy soft subsets of $K$ of the form

$$
C_{1}=T(K), C_{2}=T\left(C_{1}\right)=T^{2}(K), \ldots, C_{n}=T\left(C_{n-1}\right)=T^{n}(K)
$$

for $n \in \mathbb{N}$. It is clear that $C_{n} \widetilde{\widetilde{\subseteq}} C_{n-1}$ for each $n \in \mathbb{N}$. By proposition 40 , for each $n \in \mathbb{N}, C_{n}$ is fuzzy soft closed. Using Proposition 3.4.7, we conclude that a fuzzy soft set $D$ of the form $D=\cap_{n \in N} C_{n}$ is non empty. Observe that

$$
T(D)=T\left(\cap_{n \in N} T^{n}(K)\right) \widetilde{\widetilde{\subseteq}} \cap_{n \in N} T^{n+1}(K) \widetilde{\widetilde{\subseteq}} \cap_{n \in N} T^{n}(K)=D .
$$


Now we show that $D \widetilde{\subseteq} T(D)$. For this, suppose that there exists $F^{e} \widetilde{\in} D$ such that $F^{e}$ is not a fuzzy soft element of $T(D)$. Put $E_{n}=T^{-1}\left(F^{e}\right) \widetilde{\cap} C_{n}$. Let us observe that $E_{n} \neq \widetilde{\Phi}$ and $E_{n} \widetilde{\widetilde{E}} E_{n-1}$ for each $n \in \mathbb{N}$. By proposition 36 , there exists non empty fuzzy soft element $\digamma^{e^{\prime}} \tilde{\in} T^{-1}\left(F^{e}\right) \widetilde{\cap} D$ and thus $F^{e}=T\left(\digamma^{e^{\prime}}\right) \tilde{\in} T(D)$, a contradiction. Therefore, $T(D)=D$.

Hence the result follows using of Proposition 3.4.7. 


\section{Chapter 4}

\section{Soft Contraction Mappings}

The material of this chapter is an adaptation to the thesis of the content of the paper by Muhajid Abbas, Ghulam Murtaza and Salvador Romaguera, "Soft contraction mappings", which is accepted for publication in the JCR-journal Journal of Nonlinear and Convex Analysis 


\subsection{Introduction and preliminaries}

The notions of soft real sets, soft real numbers have several applications in real life problems [29]. These concepts paved a way to introduce soft metric spaces [28]. It is known that a soft metric space is also a soft topological space. The study of soft metric spaces and its properties is in a developing stage. On the other hand, intersection of soft set theory and fixed point theory is a very recent trend. Wardowski [128] defined the concept of a soft mappings and obtained some fixed point results in the framework of soft topological spaces. This is a beginning of a new area of research.

Fixed point results of mappings satisfying certain contractive condition on the entire domain has been at the centre of vigorous research activity. From application point of view, the situation is not completely satisfactory because it frequently happens that a mapping is a contraction not on the entire domain $X$ but merely on a subset $Y$ of $X$. However if $Y$ is closed, then it is complete and $T$ has a fixed point $x$ in $Y$ and $\left\{x_{n}\right\}$ converges to $x$ as in the case of the whole space of $X$ provided we impose some subtle restriction on the choice of initial guess $x_{0}$.

In this chapter, a concept of fixed point in soft metric spaces is studied. In order to achieve this, we first introduce the concept of soft contraction mapping on soft metric spaces and then obtain among other results, a theorem of Banach contraction principle type called soft contraction theorem in the setup of soft complete metric spaces . We also obtained a fixed point results when a soft mapping satisfies soft contraction condition on the soft closed balls in complete soft metric spaces. We provide some examples to illustrate the validity of our presented results. We believe that this will open some new avenues of research in soft metric fixed point theory.

We begin with some basic definitions and concepts related to soft metrics needed in the sequel. This section is mainly based on the definitions and results from [96], [42], [43], [41], [29] and [28]. 
In some cases, we have set our own notations, terminology and made some stylistic changes to the original definitions.

Let $U$ be a given universe, $E$ a set of parameters, and $\mathbb{R}$ the set of real numbers. Throughout this chapter, $P(U)$ and $B(\mathbb{R})$ denotes a family of all subsets of $U$ and the collection of all nonempty bounded subsets of $\mathbb{R}$, respectively..

We denote the collection of soft sets over a common universe $U$ by $S(U)$.

Definition 4.1.1 [29] If $f$ is a single valued mapping on $A \subset E$ taking values in $U$, then the pair $(f, A)$, or simply $f$, is called a soft element of $U$. Let $(F, A) \in S(U)$. A soft element $f$ of $U$ is said to belongs to $(F, A)$, denoted by $f \tilde{\in}(F, A)$, if $f(e) \in F(e)$, for each $e \in A$.

Definition 4.1.2 [29] Let $A$ be a nonempty subset of $E$. A soft real set denoted by $(\widehat{f}, A)$, or simply by $\widehat{f}$, is a mapping $\widehat{f}: A \rightarrow B(\mathbb{R})$. If $\widehat{f}$ is a single valued mapping on $A \subset E$ taking values in $\mathbb{R}$, then the pair $(\widehat{f}, A)$ or simply $\widehat{f}$, is called a soft element of $\mathbb{R}$ or a soft real number. If $\widehat{f}$ is a single valued mapping on $A \subset E$ taking values in the set $\mathbb{R}^{+}$of non negative real numbers, then a pair $(\widehat{f}, A)$, or simply $\widehat{f}$, is called a non negative soft real number. We shall denote the set of non negative soft real numbers by $\mathbb{R}(A)^{*}$. A null soft number $\overline{0}$ is a soft real number defined by $\overline{0}(e)=0$ for all $e \in A$. A unit soft number $\overline{1}$ is a soft real number defined by $\overline{1}(e)=1$ for all $e \in A$. A constant soft real number $\bar{c}$ is a soft real number such that for each $e \in A$, we have $\bar{c}(e)=c$, where $c$ is some real number.

Definition 4.1.3 [28] A soft set $(F, A)$ over $U$ is said to be a soft point if there is exactly one $e \in A$ such that $F(e)=\{x\}$ for some $x \in U$ and $F(\varepsilon)=\phi$, for all $\varepsilon \in A \backslash\{e\}$. We shall denote such a soft point by $\left(F_{\lambda}^{x}, A\right)$ or simply by $F_{e}^{x}$.

Definition 4.1.4 [28] Let $(F, A)$ be a soft set over $U$. A soft point $F_{e}^{x}$ is said to belong to $(F, A)$, denoted by $F_{e}^{x} \tilde{\in}(F, A)$, if $F_{e}^{x}(e)=\{x\} \subset F(e)$.

Definition 4.1.5 [28] Two soft points $F_{e_{1}}^{x}, F_{e_{2}}^{y}$ are said to be equal if $e_{1}=e_{2}$ and $F_{e_{1}}^{x}\left(e_{1}\right)=F_{e_{2}}^{y}\left(e_{2}\right)$, i.e., $x=y$. Thus $F_{e_{1}}^{x} \neq F_{e_{2}}^{y}$ if and only if either $x \neq y$ 
or $e_{1} \neq e_{2}$.

Proposition 4.1.6 [28] Let $(F, A)$ be a soft set over $U$. Then

$$
(F, A)=\cup\left\{F_{e}^{x}: F_{e}^{x} \tilde{\in}(F, A)\right\}
$$

Proposition 4.1.7 [28] If $(F, A),(G, A) \in S(U)$, then $(F, A) \tilde{\subset}(G, A)$ if and only if $F_{e}^{x} \tilde{\in}(F, A)$ implies that $F_{e}^{x} \tilde{\in}(G, A)$. Also, $(F, A)$ is soft equal to $(G, A)$ if and only if $F_{e}^{x} \tilde{\in}(F, A)$ if and only if $F_{e}^{x} \tilde{\in}(G, A)$.

Proposition 4.1.8 [28] For a soft point $F_{e}^{x}$, the following hold:

$$
\begin{aligned}
& F_{e}^{x} \tilde{\in}(F, A) \text { if and only if } F_{e}^{x} \tilde{\notin}(F, A)^{c}, \\
& F_{e}^{x} \tilde{\in}(F, A) \tilde{\cup}(G, A) \text { if and only if } F_{e}^{x} \tilde{\in}(F, A) \text { or } F_{e}^{x} \tilde{\in}(G, A) \text {, and } \\
& F_{e}^{x} \tilde{\in}(F, A) \tilde{\cap}(G, A) \text { if and only if } F_{e}^{x} \tilde{\in}(F, A) \text { and } F_{e}^{x} \tilde{\in}(G, A) .
\end{aligned}
$$

Remark 4.1.9 [28] Let $\mathfrak{B}$ be a collection of soft points. The soft set generated by taking all the soft points of $\mathfrak{B}$ is denoted by $S S(\mathfrak{B})$. The collection of all soft points of $(F, A)$ is denoted $S P(F, A)$.

Proposition 4.1.10 [28] Let $\mathfrak{B}, \mathfrak{B}_{1}$ and $\mathfrak{B}_{2}$ be collections of soft points, and $(F, A),(G, A) \in S(U)$. Then following hold:

$$
\begin{aligned}
S P(S S(\mathfrak{B})) & =\mathfrak{B}, S S(S P(F, A))=(F, A), \\
S P((F, A) \tilde{\cup}(G, A)) & =S P((F, A)) \cup S P((G, A)), \\
S P((F, A) \tilde{\cap}(G, A)) & =S P((F, A)) \cap S P((G, A)) \\
S S\left(\mathfrak{B}_{1} \cup \mathfrak{B}_{2}\right) & =S S\left(\mathfrak{B}_{1}\right) \tilde{\cup} S S\left(\mathfrak{B}_{2}\right) \text { and } \\
S S\left(\mathfrak{B}_{1} \cap \mathfrak{B}_{2}\right) & =S S\left(\mathfrak{B}_{1}\right) \tilde{\cap} S S\left(\mathfrak{B}_{2}\right) .
\end{aligned}
$$

Definition 4.1.11 [28] For two soft real numbers $\widehat{f}, \widehat{g}$, we say that

(i) $\widehat{f} \tilde{\leq} \widehat{g}$ if $\widehat{f}(e) \leq \widehat{g}(e)$, for all $e \in A$,

(ii) $\widehat{f} \tilde{\geq} \widehat{g}$ if $\widehat{f}(e) \geq \widehat{g}(e)$, for all $e \in A$,

(iii) $\widehat{f} \tilde{<} \widehat{g}$ if $\widehat{f}(e)<\widehat{g}(e)$, for all $e \in A$, and 
(iv) $\widehat{f} \tilde{>} \widehat{g}$ if $\widehat{f}(e)>\widehat{g}(e)$, for all $e \in A$.

The definition of a soft metric introduced in [28] is given below:

Definition 4.1.12 A mapping $d: S P(\tilde{U}) \times S P(\tilde{U}) \rightarrow \mathbb{R}(A)^{*}$ is said to be a soft metric on $\tilde{U}$ if for any $U_{\lambda}^{x}, U_{\mu}^{y}, U_{\gamma}^{z} \tilde{\in} \tilde{U}$, the following hold

M1. $d\left(U_{\lambda}^{x}, U_{\mu}^{y}\right) \tilde{\geq} \overline{0}$,

M2. $d\left(U_{\lambda}^{x}, U_{\mu}^{y}\right)=\overline{0}$ if and only if $U_{\lambda}^{x}=U_{\mu}^{y}$.

M3. $d\left(U_{\lambda}^{x}, U_{\mu}^{y}\right)=d\left(U_{\mu}^{x}, U_{\lambda}^{y}\right)$.

M4. $d\left(U_{\lambda}^{x}, U_{\gamma}^{z}\right) \tilde{\leq} d\left(U_{\lambda}^{x}, U_{\mu}^{y}\right)+d\left(U_{\mu}^{y}, U_{\gamma}^{z}\right)$.

A soft metric space is a pair $(\tilde{U}, d)$ such that $\tilde{U}$ is a soft set and $d$ is a soft metric on $\tilde{U}$.

Definition 4.1.13 [28] Let $(\tilde{U}, d)$ be a soft metric space, $\widehat{r}$ a non negative soft real number and $U_{e}^{a} \tilde{\in} \tilde{U}$. An open ball with center $U_{e}^{a}$ and radius $\widehat{r}$ is given by the set $B\left(U_{e}^{a}, \widehat{r}\right)=\left\{U_{\lambda}^{x} \tilde{\in} \tilde{U} ; d\left(U_{\lambda}^{x}, U_{e}^{a}\right) \tilde{<} \widehat{r}\right\} \subset S P(\tilde{U})$. A soft set $S S\left(B\left(U_{e}^{a}, \widehat{r}\right)\right)$ is called a soft open ball with center $U_{e}^{a}$ and radius $\widehat{r}$.

Definition 4.1.14 [28] Let $(\tilde{U}, d)$ be a soft metric space, $\widehat{r}$ a non-negative soft real number and $U_{e}^{a} \tilde{\in} \tilde{U}$. A closed ball with center $U_{e}^{a}$ and radius $\widehat{r}$ is given by a set $B\left[U_{e}^{a}, \widehat{r}\right]=\left\{U_{\lambda}^{x} \tilde{\in} \tilde{U} ; d\left(U_{\lambda}^{x}, U_{e}^{a}\right) \tilde{\leq} \widehat{r}\right\} \subset S P(\tilde{U})$. A soft set $\left.S S\left(B\left[U_{e}^{a}, \widehat{r}\right)\right]\right)$ is called a soft closed ball with center $U_{e}^{a}$ and radius $\widehat{r}$.

Definition 4.1.15 [28] Let $(F, A)$ be a soft subset in a soft metric space $(\tilde{U}, d)$. A soft point $F_{e}^{a}$ is said to be an interior point of the soft set $(F, A)$ if there exists a positive soft real number $\widehat{r}$ such that $F_{e}^{a} \in B\left(F_{e}^{a}, \widehat{r}\right) \subset$ $S P(F, A)$.

Definition 4.1.16 $[28]$ Let $(\tilde{U}, d)$ be a soft metric space and $(F, A)$ a nonnull soft subset of $\tilde{U}$. Then $(F, A)$ is soft open in $\tilde{U}$ with respect to $d$ if all soft points of $(F, A)$ are interior points of $(F, A)$.

Definition 4.1.17 $[28]$ Let $(\tilde{U}, d)$ be a soft metric space. A soft subset $(F, A)$ of $\tilde{U}$ is said to be soft closed in $\tilde{U}$ with respect to $d$ if its complement $(F, A)^{c}$ is soft open in $\tilde{U}$. 
Definition 4.1.18 [28] Let $(\tilde{U}, d)$ be a soft metric space and $(F, A) \tilde{C} \tilde{U}$. A soft point $U_{e}^{a} \tilde{\in} \tilde{U}$ is a soft limit point of $(F, A)$ if every soft open ball $S S\left(B\left(U_{e}^{a}, \widehat{r}\right)\right)$ containing $U_{e}^{a}$ contains at least one soft point of $(F, A)$ other than $U_{e}^{a}$.

Definition 4.1.19 $[28]$ Let $(\tilde{U}, d)$ be a soft metric space and $(F, A) \tilde{\subset} \tilde{U}$. Then a soft set generated by the collection of all soft points of $(F, A)$ and soft limit points of $(F, A)$ is called soft closure of $(F, A)$ in $(\tilde{U}, A)$ and is denoted by $\overline{(F, A)}$.

Definition 4.1.20 [28] Let $(\tilde{U}, d)$ be a soft metric space. A sequence $\left\{U_{\lambda, n}^{x}\right\}_{n}$ of soft points in $\tilde{U}$ is said to be convergent in $(\tilde{U}, d)$ if there is a soft point $U_{\mu}^{y} \tilde{\in} \tilde{U}$ such that $d\left(U_{\lambda, n}^{x}, U_{\mu}^{y}\right) \rightarrow \overline{0}$ as $n \rightarrow \infty$. This means for every $\widehat{\varepsilon} \tilde{>0}$, chosen arbitrary, there exists a natural number $N=N(\tilde{\varepsilon})$ such that $d\left(U_{\lambda, n}^{x}, U_{\mu}^{y}\right) \tilde{<} \widehat{\varepsilon}$, whenever $n>N$.

Proposition 4.1.21 [28] Limit of a sequence $\left\{U_{\lambda, n}^{x}\right\}_{n}$ in a soft metric space $(\tilde{U}, d)$, if exists is unique.

Proposition 4.1.22 [28] Let $(\tilde{U}, d)$ be a soft metric space and $(F, A) \tilde{\subset} \tilde{U}$. Then $U_{\mu}^{y} \tilde{\in} \tilde{U}$ is a soft limit point of $(F, A)$ if and only if there is a sequence $\left\{U_{\lambda, n}^{x}\right\}_{n}$ in $(F, A)$ other than $\left\{U_{\mu}^{y}\right\}_{n}$ which converges to $U_{\mu}^{y}$.

Proposition 4.1.23 [28] Let $(F, A)$ be a soft subset in a soft metric space $(\tilde{U}, d)$. Then $(F, A)$ is soft closed if and only if $\left\{U_{\lambda, n}^{x}\right\}_{n}$ in $(F, A)$ which converges in $\tilde{U}$ cannot converges to a soft point of $(F, A)^{c}$.

Definition 4.1.24 [28] A sequence $\left\{U_{\lambda, n}^{x}\right\}_{n}$ of soft points in $(\tilde{U}, d)$ is said to be a Cauchy sequence in $(\tilde{U}, d)$ if corresponding to every $\widehat{\varepsilon} \tilde{>} \overline{0}$, there exists a natural number $m$ such that $d\left(U_{\lambda, i}^{x}, U_{\lambda, j}^{x}\right) \tilde{<} \widehat{\varepsilon}$, for all $i, j \geq m$. That is, $d\left(U_{\lambda, i}^{x}, U_{\lambda, j}^{x}\right) \rightarrow \overline{0}$ as $i, j \rightarrow \infty$.

Proposition 4.1.25 [28] Every convergent sequence $\left\{U_{\lambda, n}^{x}\right\}_{n}$ in a soft metric space $(\tilde{U}, d)$ is Cauchy and every Cauchy sequence is bounded.

Definition 4.1.26 [28] A soft metric space $(\tilde{U}, d)$ is called complete if every Cauchy sequence in $\tilde{U}$ converges to some soft point of $(\tilde{U}, d)$. In this case, we say that the soft metric $d$ is complete. 


\subsection{Fixed point theorems}

In the sequel, soft real numbers will be denoted with "tildes" instead of "widehats", i.e., we will write $\tilde{\varepsilon}, \tilde{\delta}$, etc., instead of $\widehat{\varepsilon}, \widehat{\delta}$, etc.

Now we prove the following proposition.

Proposition 4.2.1 A soft subset $(F, A)$ of a soft complete metric space $(\tilde{U}, d)$ is soft complete if and only if $(F, A)$ is soft closed in $(\tilde{U}, d)$.

Proof. Suppose that $(F, A)$ is soft complete. By Proposition 4.1.22, we know that for every $F_{\mu}^{y} \tilde{\in} \overline{(F, A)}$, there is a sequence $\left\{F_{\lambda, n}^{x}\right\}_{n}$ in $(F, A)$ which converges to $F_{\mu}^{y}$. As $\left\{F_{\lambda, n}^{x}\right\}_{n}$ is a Cauchy sequence (Proposition 4.1.34) and $(F, A)$ is soft complete, $\left\{F_{\lambda, n}^{x}\right\}_{n}$ converges in $(F, A)$. By uniqueness of the limit (Proposition 4.1.21) we obtain that $F_{\mu}^{y} \tilde{\in}(F, A)$. This proves that $(F, A)$ is soft closed. Conversely, if $(F, A)$ is soft closed and $\left\{F_{\lambda, n}^{x}\right\}_{n}$ is Cauchy sequence in $(F, A)$. Then by the completeness of $(\tilde{U}, d)$ we have $F_{\lambda, n}^{x} \rightarrow F_{\mu}^{y} \tilde{\in} \tilde{U}$, which by Proposition 4.1.23, further implies that $F_{\mu}^{y} \tilde{\in}(F, A)$. Hence $(F, A)$ is soft complete.

The following definitions are somehow similar to those given by Wardowski ([128]).

Definition 4.2.2 Let $(F, A),(G, A) \in S(U)$. The soft Cartesian product of $(F, A)$ and $(G, A)$, denoted by $(F, A) \tilde{\times}(G, A)$, is defined as

$$
(F, A) \tilde{\times}(G, A)=\left\{\left(\left(p_{1}, p_{2}\right), F\left(p_{1}\right) \times G\left(p_{2}\right)\right): p_{1}, p_{2} \in A\right\}
$$

Example 4.2.3 Suppose that $U=\left\{h_{1}, h_{2}, h_{3}\right\}$ and $A=\left\{p_{1}, p_{2}, p_{3}\right\}$. Define soft sets $(F, A)$ and $(G, A)$ as follows:

$$
\begin{aligned}
& (F, A)=\left\{\left(p_{1},\left\{h_{1}, h_{2}\right\}\right),\left(p_{2},\left\{h_{2}, h_{3}\right\}\right),\left(p_{3},\left\{h_{1}\right\}\right)\right\} \text { and } \\
& (G, A)=\left\{\left(p_{1},\left\{h_{1}\right\}\right),\left(p_{2},\left\{h_{1}, h_{3}\right\}\right),\left(p_{3},\left\{h_{1}, h_{2}\right\}\right)\right\} .
\end{aligned}
$$


Then

$$
\begin{aligned}
& (F, A) \tilde{\times}(G, A) \\
= & \left\{\left(\left(p_{1}, p_{1}\right),\left\{h_{1}, h_{2}\right\} \times\left\{h_{1}\right\}\right),\left(\left(p_{1}, p_{2}\right),\left\{h_{1}, h_{2}\right\} \times\left\{h_{1}, h_{3}\right\}\right),\right. \\
& \left(\left(p_{1}, p_{3}\right),\left\{h_{1}, h_{2}\right\} \times\left\{h_{1}, h_{2}\right\}\right),\left(\left(p_{2}, p_{1}\right),\left\{h_{2}, h_{3}\right\} \times\left\{h_{1}\right\}\right), \\
& \left(\left(p_{2}, p_{2}\right),\left\{h_{2}, h_{3}\right\} \times\left\{h_{1}, h_{3}\right\}\right),\left(\left(p_{2}, p_{3}\right),\left\{h_{2}, h_{3}\right\} \times\left\{h_{1}, h_{2}\right\}\right), \\
& \left(\left(p_{3}, p_{1}\right),\left\{h_{1}\right\} \times\left\{h_{1}\right\}\right),\left(\left(p_{3}, p_{2}\right),\left\{h_{1}\right\} \times\left\{h_{1}, h_{3}\right\}\right), \\
& \left.\left(\left(p_{3}, p_{3}\right),\left\{h_{1}\right\} \times\left\{h_{1}, h_{2}\right\}\right)\right\} .
\end{aligned}
$$

Definition 4.2.4 Let $(F, A),(G, A)$ be two soft sets over a common universe $U$. A soft relation $R$ is a soft set such that $(R, A \times A) \tilde{\subset}(F, A) \tilde{\times}(G, A)$, i.e., $(R, A \times A)=\left\{\left((p, q), U_{p} \times U_{q}\right): p, q \in A, U_{p} \subseteq F(p), U_{q} \subseteq G(q)\right\}$.

We will denote $\left((p, q), U_{p} \times U_{q}\right) \in(R, A \times A)$ as $\left(p, U_{p}\right) R\left(q, U_{q}\right)$.

Example 4.2.5 Let $(F, A),(G, A)$ be as in Example 4.2.3. Then $R=\left\{\left(\left(p_{1}, p_{1}\right),\left\{\left(h_{1}, h_{1}\right)\right\}\right),\left(\left(p_{2}, p_{1}\right),\left\{\left(h_{2}, h_{1}\right)\right\}\right),\left(\left(p_{2}, p_{3}\right),\left\{\left(h_{2}, h_{1}\right),\left(\left(h_{3}, h_{2}\right)\right)\right\}\right)\right\}$.

So we can write

$$
\left(p_{1},\left\{h_{1}\right\}\right) R\left(p_{1},\left\{h_{1}\right\}\right),\left(p_{2},\left\{h_{2}\right\}\right) R\left(p_{1},\left\{h_{1}\right\}\right),\left(p_{2},\left\{h_{2}, h_{3}\right\}\right) R\left(p_{3},\left\{h_{1}, h_{2}\right\}\right) .
$$

Definition 4.2.6 Let $(F, A)$ and $(G, A)$ be two soft sets. A soft relation $(T, A \times A) \tilde{\subset}(F, A) \tilde{\times}(G, A)$ is called a soft mapping from $(F, A)$ to $(G, A)$ if for each soft point $F_{\lambda}^{x} \tilde{\in}(F, A)$ there exists only one soft point $F_{\mu}^{y}$ such that $F_{\lambda}^{x} T F_{\mu}^{y}$. We will denote $F_{\lambda}^{x} T F_{\mu}^{y}$ by $T\left(F_{\lambda}^{x}\right)=F_{\mu}^{y}$. If $(T, A \times A) \tilde{\subset}(F, A) \tilde{\times}(G, A)$ is soft mapping from $(F, A)$ to $(G, A)$, then we write it as $T:(F, A) \stackrel{\sim}{\rightarrow}(G, A)$.

Example 4.2.7 Let $(F, A),(G, A)$ be as in Example 4.2.3. Suppose that $(T, A \times A) \tilde{\subset}(F, A) \tilde{\times}(G, A)$ is defined as:

$$
\begin{aligned}
T= & \left\{\left(p_{1}, p_{3}\right),\left\{\left(h_{1}, h_{2}\right)\right\}\right),\left(\left(p_{1}, p_{2}\right),\left\{\left(h_{2}, h_{1}\right)\right\}\right),\left(\left(p_{2}, p_{1}\right),\left\{\left(h_{3}, h_{1}\right)\right\}\right), \\
& \left.\left(\left(p_{2}, p_{2}\right),\left\{\left(h_{2}, h_{1}\right)\right\}\right),\left(\left(p_{3}, p_{2}\right),\left\{\left(h_{1}, h_{3}\right)\right\}\right)\right\} .
\end{aligned}
$$


Therefore we can write $T\left(F_{p_{1}}^{h_{1}}\right)=F_{p_{3}}^{h_{2}}, T\left(F_{p_{1}}^{h_{2}}\right)=F_{p_{2}}^{h_{1}}, T\left(F_{p_{2}}^{h_{3}}\right)=F_{p_{1}}^{h_{1}}$, $T\left(F_{p_{2}}^{h_{2}}\right)=F_{p_{2}}^{h_{1}}$ and $T\left(F_{p_{3}}^{h_{1}}\right)=F_{p_{2}}^{h_{3}}$.

Definition 4.2.8 Let $(F, A)$ and $(G, A)$ be two soft sets and $T:(F, A) \stackrel{\sim}{\rightarrow}(G, A)$ a soft mapping. The image of $(H, A) \tilde{C}(F, A)$ under the soft mapping $T$ is the soft set, denoted by $T((H, A))$, defined as follows

$$
T((H, A))=\tilde{\cup}\left\{T\left\{F_{\lambda}^{x}\right\}: F_{\lambda}^{x} \tilde{\in}(H, A)\right\} .
$$

Definition 4.2.9 Let $(F, A)$ and $(G, A)$ be two soft sets and $T:(F, A) \stackrel{\sim}{\rightarrow}(G, A)$ a soft mapping. The inverse of $(Y, A) \tilde{C}(G, A)$-under the soft mapping $T$ is the soft set, denoted by $T^{-1}((Y, A))$, defined as:

$$
T^{-1}((Y, A))=\tilde{\cup}\left\{\left\{F_{\lambda}^{x}\right\}: F_{\lambda}^{x} \tilde{\in} F, T\left\{F_{\lambda}^{x}\right\} \tilde{\in} Y\right\} .
$$

Definition 4.2.10 Let $(F, A)$ be a soft set and $T:(F, A) \stackrel{\sim}{\rightarrow}(F, A)$ a soft mapping. A soft point $F_{\lambda}^{x} \tilde{\in}(F, A)$ is called a fixed point of $T$ if $T\left(F_{\lambda}^{x}\right)=F_{\lambda}^{x}$. Example 4.2.11 Let $U=\left\{h_{1}, h_{2}, h_{3}\right\}, A=\left\{p_{1}, p_{2}\right\}$. Define the soft set $(F, A)$ as follows

$$
(F, A)=\left\{\left(p_{1},\left\{h_{1}, h_{2}\right\}\right),\left(p_{2},\left\{h_{2}, h_{3}\right\}\right)\right\}
$$

If $T:(F, A) \stackrel{\sim}{\rightarrow}(F, A)$ is defined as:

$$
T\left(F_{p_{1}}^{h_{1}}\right)=F_{p_{1}}^{h_{1}}, T\left(F_{p_{1}}^{h_{2}}\right)=F_{p_{2}}^{h_{2}}, T\left(F_{p_{2}}^{h_{2}}\right)=F_{p_{2}}^{h_{3}}, \text { and } T\left(F_{p_{2}}^{h_{3}}\right)=F_{p_{1}}^{h_{2}}
$$

then $F_{p_{1}}^{h_{1}}$ is the fixed point of $T$.

Definition 4.2.12 Let $\left(\tilde{U}_{1}, d_{1}\right)$ and $\left(\tilde{U}_{2}, d_{2}\right)$ be two soft metric spaces. A soft mapping $T: \tilde{U}_{1} \tilde{\rightarrow}_{2}$ is said to be soft continuous at a soft point $U_{\lambda}^{x} \tilde{\in} \tilde{U}$ if for every $\tilde{\varepsilon} \tilde{>} \overline{0}$, there is a $\tilde{\delta} \tilde{>} \overline{0}$ such that $d_{2}\left(T\left(U_{\lambda}^{x}\right), T\left(U_{\mu}^{y}\right)\right) \tilde{<} \tilde{\varepsilon}$ whenever $d_{1}\left(U_{\lambda}^{x}, U_{\mu}^{y}\right) \tilde{<} \tilde{\delta}$. If $T$ is soft continuous at every soft point of $\tilde{U}$, we say that $T$ is soft continuous on $\tilde{U}$.

Proposition 4.2.13 Let $\left(\tilde{U}, d_{1}\right)$ and $\left(\check{Y}, d_{2}\right)$ be two soft metric spaces. For a soft mapping $T: \tilde{U} \stackrel{\sim}{\rightarrow} \check{Y}$ the following are equivalent: 
(i) $T$ is soft continuous on $\tilde{U}$.

(ii) For any $U_{\mu}^{y} \tilde{\in} \tilde{U}$, if $U_{\lambda, n}^{x} \rightarrow U_{\mu}^{y}$ in $\tilde{U}$, then $T\left(U_{\lambda, n}^{x}\right) \rightarrow T\left(U_{\mu}^{y}\right)$ in $\check{Y}$.

(iii) If $(W, A)$ is soft closed in $\check{Y}$, then $T^{-1}(W, A)$ is soft closed in $\tilde{U}$.

(iv) If $(V, A)$ is soft open in $\check{Y}$, then $T^{-1}(V, A)$ is soft open in $\tilde{U}$.

Proof. $\quad($ i $) \Rightarrow\left(\right.$ ii): Suppose that $U_{\lambda, n}^{x} \rightarrow U_{\mu}^{y}$ in $\tilde{U}$. Given $\tilde{\varepsilon} \tilde{>} \overline{0}$, let $\tilde{\delta} \tilde{>} \overline{0}$ such that $T\left(S S\left(B\left(U_{\mu}^{y}, \tilde{\delta}\right)\right)\right) \tilde{\subset} S S\left(B\left(T\left(U_{\mu}^{y}\right), \tilde{\varepsilon}\right)\right)$. Then, since $U_{\lambda, n}^{x} \rightarrow U_{\mu}^{y}$, we have $\left\{U_{\lambda, n}^{x}\right\}_{n}$ eventually in $S S\left(B\left(U_{\mu}^{y}, \tilde{\delta}\right)\right)$. But this implies that $\left\{T\left(U_{\lambda, n}^{x}\right)\right\}_{n}$ eventually in $S S\left(B\left(T\left(U_{\mu}^{y}\right), \tilde{\varepsilon}\right)\right)$. Since $\tilde{\varepsilon}$ is arbitrary, this means that $T\left(U_{\lambda, n}^{x}\right) \rightarrow$ $T\left(U_{\mu}^{y}\right)$.

(ii) $\Rightarrow$ (iii) Let $(W, A)$ be soft closed in $\check{Y}$. Given $\left\{U_{\lambda, n}^{x}\right\}_{n}$ in $T^{-1}(W, A)$ such that $U_{\lambda, n}^{x} \rightarrow U_{\mu}^{y}$ in $\tilde{U}$, we are to show that $U_{\mu}^{y} \tilde{\in} T^{-1}(W, A)$. But $\left\{U_{\lambda, n}^{x}\right\}_{n}$ in $T^{-1}(W, A)$ implies that $\left\{T\left(U_{\lambda, n}^{x}\right)\right\}_{n}$ is in $(W, A)$, while $U_{\lambda, n}^{x} \rightarrow U_{\mu}^{y}$ in $\tilde{U}$ tells us that $T\left(U_{\lambda, n}^{x}\right) \rightarrow T\left(U_{\mu}^{y}\right)$ in $\check{Y}$ from (ii). Thus, since $(W, A)$ is soft closed, we have that $T\left(U_{\mu}^{y}\right) \tilde{\epsilon}(W, A)$ or $U_{\mu}^{y} \tilde{\in} T^{-1}(W, A)$.

(iii) $\Leftrightarrow$ (iv) It is obvious, since $T^{-1}\left((V, A)^{c}\right)=\left(T^{-1}(V, A)\right)^{c}$.

(iv) $\Rightarrow$ (i) Given $U_{\lambda}^{x} \tilde{\in} \tilde{U}$ and $\tilde{\varepsilon} \tilde{>} \overline{0}$, the set $S S\left(B\left(T\left(U_{\lambda}^{x}\right), \tilde{\varepsilon}\right)\right)$ is open in $\check{Y}$ and thus, by (iv), the set $T^{-1}\left(S S\left(B\left(T\left(U_{\lambda}^{x}\right), \tilde{\varepsilon}\right)\right)\right)$ is open in $\tilde{U}$.

Therefore, $S S\left(B\left(U_{\lambda}^{x}, \tilde{\delta}\right)\right) \tilde{\subset} T^{-1}\left(S S\left(B\left(T\left(U_{\lambda}^{x}\right), \tilde{\varepsilon}\right)\right)\right)$ for some $\tilde{\delta} \tilde{>} \overline{0}$, because $U_{\lambda}^{x} \tilde{\in} S S\left(B\left(T\left(U_{\lambda}^{x}\right), \tilde{\varepsilon}\right)\right)$.

Definition 4.2.14 Let $(\tilde{U}, d)$ be a soft metric space and $T: \tilde{U} \tilde{\rightarrow} \tilde{U}$ a soft mapping. Then $T$ is said to be a soft contraction if

$$
d\left(T\left(U_{\lambda}^{x}\right), T\left(U_{\mu}^{y}\right)\right) \tilde{\leq} \bar{c} d\left(U_{\lambda}^{x}, U_{\mu}^{y}\right)
$$

for all $U_{\lambda}^{x}, U_{\mu}^{y} \in S U(\tilde{U})$, where $\overline{0} \leq \bar{c}<\overline{1}$. We will call $\bar{c}$ as soft contraction constant.

Remark 4.2.15 A soft contraction on a soft metric space is a soft continuous mapping.

Our main result is the following. 
Theorem 4.2.16 Let $(\tilde{U}, d)$ be a soft complete metric space, where $d$ : $S P(\tilde{U}) \times S P(\tilde{U}) \rightarrow \mathbb{R}(A)^{*}$ with $A$ a (non-empty) finite set, and let $T$ be a soft contraction with soft contraction constant $\bar{c}$. Then $T$ has unique soft fixed point $U_{\mu}^{y}$. Moreover, for any soft point $U_{\lambda}^{x}$, the sequence of iterates $\left\{T^{n}\left(U_{\lambda}^{x}\right)\right\}_{n}$ converges to $U_{\mu}^{y}$, and the following hold:

$$
\begin{aligned}
d\left(U_{\lambda, n}^{x}, U_{\lambda, m}^{x}\right) & \tilde{\leq} \frac{\bar{c}^{m}}{1-\bar{c}} d\left(T\left(U_{\lambda, 0}^{x}\right), U_{\lambda, 0}^{x}\right), \text { whenever } n>m \\
d\left(U_{\lambda, m}^{x}, U_{\mu}^{y}\right) & \tilde{\leq} \bar{c} d\left(U_{\lambda, m-1}^{x}, U_{\mu}^{y}\right),
\end{aligned}
$$

and

$$
d\left(U_{\lambda, m}^{x}, U_{\mu}^{y}\right) \tilde{\leq} \frac{\bar{c}}{1-\bar{c}} d\left(U_{\lambda, m-1}^{x}, U_{\lambda, m}^{x}\right)
$$

where $U_{\lambda, 0}^{x}=U_{\lambda}^{x}$ and $U_{\lambda, n+1}^{x}=T\left(U_{\lambda, n}^{x}\right)$ for all $n=0,1,2 \ldots$

Proof Choose a soft point $U_{\lambda}^{x}$. Put $U_{\lambda, 0}^{x}=U_{\lambda}^{x}$ and note that

$$
\begin{aligned}
d\left(U_{\lambda, n+1}^{x}, U_{\lambda, n}^{x}\right) & =d\left(T\left(U_{\lambda, n}^{x}\right), T\left(U_{\lambda, n-1}^{x}\right)\right) \tilde{\leq} \bar{c} d\left(U_{\lambda, n}^{x}, U_{\lambda, n-1}^{x}\right) \\
& \tilde{\leq} \bar{c}^{2} d\left(U_{\lambda, n-1}^{x}, U_{\lambda, n-2}^{x}\right) \tilde{\leq} \\
\cdots & \tilde{\leq} \bar{c}^{n} d\left(U_{\lambda, 1}^{x}, U_{\lambda, 0}^{x}\right) .
\end{aligned}
$$

For $n>m$, we have

$$
\begin{aligned}
d\left(U_{\lambda, n}^{x}, U_{\lambda, m}^{x}\right) & \tilde{\leq} d\left(U_{\lambda, n}^{x}, U_{\lambda, n-1}^{x}\right)+d\left(U_{\lambda, n-1}^{x}, U_{\lambda, n-2}^{x}\right)+\cdots+d\left(U_{\lambda, m+1}^{x}, U_{\lambda, m}^{x}\right) \\
& \tilde{\leq}\left(\bar{c}^{n-1}+\bar{c}^{n-2}+\cdots+\bar{c}^{m}\right) d\left(U_{\lambda, 1}^{x}, U_{\lambda, 0}^{x}\right) \\
& \tilde{\leq} \frac{\bar{c}^{m}}{1-\bar{c}} d\left(U_{\lambda, 1}^{x}, U_{\lambda, 0}^{x}\right) \cdot 4.2 .1
\end{aligned}
$$

Now we show that $\left\{U_{\lambda, n}^{x}\right\}_{n}$ is a Cauchy sequence. Indeed, choose an arbitrary soft real number $\widetilde{\varepsilon}>\overline{0}$. Since $A$ is finite, we can write $A=\left\{\lambda_{1}, \ldots, \lambda_{k}\right\}$. Then, for each $i \in\{1, \ldots, k\}$, there exists an $N_{i} \in \mathbb{N}$ such that

$$
\left(\frac{\bar{c}^{N_{i}}}{1-\bar{c}} d\left(U_{\lambda, 1}^{x}, U_{\lambda, 0}^{x}\right)\right)\left(\lambda_{i}\right)<\widetilde{\varepsilon}\left(\lambda_{i}\right)
$$


Take $N=\max \left\{N_{1}, \ldots, N_{k}\right\}$. Therefore, for any $n>m \geq N$ and any $i \in$ $\{1, \ldots, k\}$, we have

$$
\begin{aligned}
d\left(U_{\lambda, n}^{x}, U_{\lambda, m}^{x}\right)\left(\lambda_{i}\right) & \leq\left(\frac{\bar{c}^{m}}{1-\bar{c}} d\left(U_{\lambda, 1}^{x}, U_{\lambda, 0}^{x}\right)\right)\left(\lambda_{i}\right) \\
& \leq\left(\frac{\bar{c}^{N}}{1-\bar{c}} d\left(U_{\lambda, 1}^{x}, U_{\lambda, 0}^{x}\right)\right)\left(\lambda_{i}\right)<\widetilde{\varepsilon}\left(\lambda_{i}\right)
\end{aligned}
$$

i.e.,

$$
d\left(U_{\lambda, n}^{x}, U_{\lambda, m}^{x}\right) \tilde{<} \widetilde{\varepsilon}
$$

whenever $n>m \geq N$. Hence $\left\{U_{\lambda, n}^{x}\right\}_{n}$ is a Cauchy sequence. By the completeness of $(\tilde{U}, d)$ there is a $U_{\mu}^{y} \tilde{\in} \tilde{U}$ such that $d\left(U_{\lambda, n}^{x}, U_{\mu}^{y}\right) \rightarrow \overline{0}$ as $n \rightarrow \infty$.

Since

$$
\begin{aligned}
d\left(U_{\mu}^{y}, T\left(U_{\mu}^{y}\right)\right) & \tilde{\leq} d\left(U_{\lambda, n}^{x}, U_{\mu}^{y}\right)+d\left(U_{\lambda, n}^{x}, T\left(U_{\mu}^{y}\right)\right) \\
& \tilde{\leq} d\left(U_{\lambda, n}^{x}, U_{\mu}^{y}\right)+\bar{c} d\left(U_{\lambda, n-1}^{x}, U_{\mu}^{y}\right)
\end{aligned}
$$

we can make the second term smaller than any $\tilde{\varepsilon} \tilde{>} \overline{0}$ as $U_{\lambda, n}^{x} \rightarrow U_{\mu}^{y}$. Hence $d\left(U_{\mu}^{y}, T\left(U_{\mu}^{y}\right)\right)=\overline{0}$. This implies $T\left(U_{\mu}^{y}\right)=U_{\mu}^{y}$. So $U_{\mu}^{y}$ is a fixed point of $T$.

Now if $U_{\gamma}^{z}$ is another fixed point of $T$, then

$$
d\left(U_{\mu}^{y}, U_{\gamma}^{z}\right)=d\left(T\left(U_{\mu}^{y}\right), T\left(U_{\gamma}^{z}\right)\right) \tilde{\leq} \bar{c} d\left(U_{\mu}^{y}, U_{\gamma}^{z}\right)
$$

implies that $d\left(U_{\mu}^{y}, U_{\gamma}^{z}\right)=\overline{0}$ as $\bar{c} \tilde{<} \overline{1}$. Hence $U_{\mu}^{y}=U_{\gamma}^{z}$. Therefore the fixed point of $T$ is unique.

As for $n>m$, we have

$$
\begin{aligned}
d\left(U_{\lambda, n}^{x}, U_{\lambda, m}^{x}\right) & \tilde{\leq} \frac{\bar{c}^{m}}{1-\bar{c}} d\left(U_{\lambda, 1}^{x}, U_{\lambda, 0}^{x}\right) \\
& =\frac{\bar{c}^{m}}{1-\bar{c}} d\left(T\left(U_{\lambda, 0}^{x}\right), U_{\lambda, 0}^{x}\right) .
\end{aligned}
$$

Taking limit as $n \rightarrow \infty$, we obtain that

$$
\begin{aligned}
d\left(U_{\lambda, m}^{x}, U_{\mu}^{y}\right) & =d\left(T\left(U_{\lambda, m-1}^{x}\right), T\left(U_{\mu}^{y}\right)\right) \\
& \tilde{\leq} \bar{c} d\left(U_{\lambda, m-1}^{x}, U_{\mu}^{y}\right) \\
& \tilde{\leq} \bar{c}\left[d\left(U_{\lambda, m-1}^{x}, U_{\lambda, m}^{x}\right)+d\left(U_{\lambda, m}^{x}, U_{\mu}^{y}\right)\right]
\end{aligned}
$$


This implies

$$
d\left(U_{\lambda, m}^{x}, U_{\mu}^{y}\right) \tilde{\leq} \frac{\bar{c}}{1-\bar{c}} d\left(U_{\lambda, m-1}^{x}, U_{\lambda, m}^{x}\right)
$$

Remark 4.2.17 When $T: \tilde{U} \stackrel{\sim}{\rightarrow}$ is a soft contraction with constant $\bar{c}$, any iterate $T^{n}$ is a soft contraction with constant $\bar{c}^{n}$. The unique soft fixed point of $T$ will also be the unique soft fixed point of $T^{n}$.

Corollary 4.2.18 Let $(\tilde{U}, d)$ be a soft complete metric space, where $d$ : $S P(\tilde{U}) \times S P(\tilde{U}) \rightarrow \mathbb{R}(A)^{*}$ with $A$ a (non-empty) finite set, $T: \tilde{U} \stackrel{\sim}{\rightarrow}$ a soft contraction and $Y \tilde{\subset} \tilde{U}$ a soft closed subset such that $T(Y) \tilde{\subset} Y$. Then the unique soft fixed point of $T$ is a soft point of $Y$.

Proof. Since $Y$ is a soft closed subset of a soft complete metric space, it is soft complete. Then by applying soft contraction mapping theorem to $T$ on $Y$, we obtain a soft fixed point of $T$ in $Y$. Since $T$ has only one fixed point in $\tilde{U}$, it must lie in $Y$.

Theorem 4.2.19 Let $T$ be a soft mapping on a soft complete metric space $(\tilde{U}, d)$, where $d: S P(\tilde{U}) \times S P(\tilde{U}) \rightarrow \mathbb{R}(A)^{*}$ with $A$ a (non-empty) finite set . Suppose $T$ is a soft contraction on a soft closed ball $S S\left(B\left[U_{\lambda, 0}^{x}, \tilde{r}\right]\right)$ with soft contraction constant $\bar{c}$ and $d\left(T\left(U_{\lambda, 0}^{x}\right), U_{\lambda, 0}^{x}\right) \tilde{<}(1-\bar{c}) \tilde{r}$. Then $T$ has a unique soft fixed point in $S S\left(B\left[U_{\lambda, 0}^{x}, \tilde{r}\right]\right)$.

Proof. Construct a sequence $\left\{U_{\lambda, m}^{x}\right\}_{m}$ as in the previous theorem starting from $U_{\lambda, 0}^{x}$. Now taking $m=0$ in (4.2.1) and changing $n$ to $m$, we have

$$
\begin{aligned}
d\left(U_{\lambda, m}^{x}, U_{\lambda, 0}^{x}\right) & \tilde{\leq} \frac{1}{1-\bar{c}} d\left(U_{\lambda, 1}^{x}, U_{\lambda, 0}^{x}\right) \\
& =\frac{1}{1-\bar{c}} d\left(T\left(U_{\lambda, 0}^{x}\right), U_{\lambda, 0}^{x}\right) \tilde{<} \tilde{r} .
\end{aligned}
$$

Hence all $U_{\lambda, m}^{x}$ 's are in $S S\left(B\left[U_{\lambda, 0}^{x}, \tilde{r}\right]\right)$. Since $\left\{U_{\lambda, m}^{x}\right\}_{m}$ is a Cauchy sequence, by the completeness of $(\tilde{U}, d)$ we have $U_{\lambda, m}^{x} \rightarrow U_{\mu}^{y} \tilde{\in} \tilde{U}$. As $S S\left(B\left[U_{\lambda, 0}^{x}, \tilde{r}\right]\right)$ is soft closed, so $U_{\mu}^{y} \tilde{\in} S S\left(B\left[U_{\lambda, 0}^{x}, \tilde{r}\right]\right)$. Hence the result.

We conclude the chapter with two examples that illustrate our main result. They are based on the following interesting example given in [28, Example 4.3]. 
Example 4.2.20 Let $U$ and $A$ be non-empty subsets of $\mathbb{R}$. Define $d$ : $S P(\tilde{U}) \times S P(\tilde{U}) \rightarrow \mathbb{R}(A)^{*}$ as

$$
d\left(U_{\lambda}^{x}, U_{\mu}^{y}\right)=|\bar{x}-\bar{y}|+|\bar{\lambda}-\bar{\mu}|
$$

for all $U_{\lambda}^{x}, U_{\mu}^{y} \in \tilde{U}$, where $|$.$| denotes the modulus of soft real numbers (recall$ that for each $x \in U, \bar{x}$ is the constant soft real number defined by $\bar{x}(\lambda)=x$ for all $\lambda \in A$, and, similarly, for each $\lambda \in A, \bar{\lambda}$ is the constant soft real number defined by $\bar{\lambda}(\mu)=\lambda$ for all $\mu \in A$ ). Then, according to [28, Example 4.3], $d$ is a soft metric on $\tilde{U}$.

Example 4.2.21 Let $U=[0,1]$ and $E=A=\{0,1\}$. Let $d$ be the soft metric on $\widetilde{U}$ as constructed in Example 4.2.20. Since $U$ is complete for the Euclidean metric, it immediately follows that $(\tilde{U}, d)$ is complete.

Now define a soft mapping $T: \tilde{U} \rightarrow \tilde{U}$ as $T\left(U_{0}^{x}\right)=U_{0}^{0}$ and $T\left(U_{1}^{x}\right)=U_{0}^{x / 2}$ for all $x \in U$. We show that $T$ is a soft contraction with soft contraction constant $\bar{c}$ given by $\bar{c}(0)=\bar{c}(1)=1 / 2$. Indeed, for each $x, y \in U$ we have $d\left(T\left(U_{0}^{x}\right), T\left(U_{0}^{y}\right)\right)=\overline{0}$, and

$$
d\left(T\left(U_{1}^{x}\right), T\left(U_{1}^{y}\right)\right)=d\left(U_{0}^{x / 2}, U_{0}^{y / 2}\right)=|\overline{x / 2}-\overline{y / 2}| \widetilde{\leq} \bar{c}|\bar{x}-\bar{y}| .
$$

Finally, since $x, y \in[0,1]$, one has for $\mu=0,1$,

$$
|\overline{y / 2}-\overline{0}|(\mu)=y / 2 \leq(|x-y|+1) / 2=(\bar{c}(|\bar{x}-\bar{y}|+|\overline{1}-\overline{0}|))(\mu),
$$

SO

$d\left(T\left(U_{0}^{x}\right), T\left(U_{1}^{y}\right)\right)=d\left(U_{0}^{0}, U_{0}^{y / 2}\right)=|\overline{y / 2}-\overline{0}| \tilde{\leq} \bar{c}(|\bar{x}-\bar{y}|+|\overline{1}-\overline{0}|)=\bar{c} d\left(U_{0}^{x}, U_{1}^{y}\right)$.

Consequently, all conditions of Theorem 4.2.16 are satisfied. In fact, $U_{0}^{0}$ is the unique fixed point of $T$.

Our last example shows that condition " $A$ is a finite set" cannot be omitted in Theorem 4.2.16.

Example 4.2.22 Let $U=A=\{1 / n: n \in \mathbb{N}\}$. Let $d$ be the soft metric on $\widetilde{U}$ as constructed in Example 4.2.20. We show that $(\widetilde{U}, d)$ is complete. 
Indeed, suppose that $\left\{U_{\lambda, n}^{x}\right\}_{n}$ is a Cauchy sequence in $(\widetilde{U}, d)$. Take the soft real number $\widetilde{\varepsilon}$ such that $\widetilde{\varepsilon}(\lambda)=\lambda$ for all $\lambda \in A$, i.e., $\widetilde{\varepsilon}(1 / k)=1 / k$ for all $k \in \mathbb{N}$. Then, there is $m \in \mathbb{N}$ such that

$$
d\left(U_{\lambda, i}^{x}, U_{\lambda, j}^{x}\right) \tilde{<} \widetilde{\varepsilon}
$$

for all $i, j \geq m$. This implies that $d\left(U_{\lambda, i}^{x}, U_{\lambda, j}^{x}\right)(1 / k)<\widetilde{\varepsilon}(1 / k)$ for all $k \in \mathbb{N}$. Hence

$$
\left(\left|\overline{x_{i}}-\overline{x_{j}}\right|+\left|\overline{\lambda_{i}}-\overline{\lambda_{j}}\right|\right)\left(\frac{1}{k}\right)<\frac{1}{k},
$$

for all $i, j \geq m$ and for all $k \in \mathbb{N}$. Consequently

$$
\left|x_{i}-x_{j}\right|+\left|\lambda_{i}-\lambda_{j}\right|<\frac{1}{k}
$$

for all $i, j \geq m$ and for all $k \in \mathbb{N}$. In particular, for any $j \geq m$,

$$
\left|x_{j}-x_{j+1}\right|+\left|\lambda_{j}-\lambda_{j+1}\right|<\frac{1}{k}
$$

for all $k \in \mathbb{N}$.

Therefore $x_{j}=x_{j+1}$ and $\lambda_{j}=\lambda_{j+1}$ for all $j \geq m$. We deduce that $x_{j}=x_{m}$ and $\lambda_{j}=\lambda_{m}$ for all $j \geq m$. Thus the sequence $\left\{U_{\lambda, n}^{x}\right\}_{n}$ is eventually constant, and hence convergent. We conclude that $(\widetilde{U}, d)$ is complete.

Now let $T: \widetilde{U} \rightarrow \widetilde{U}$ defined as $T\left(U_{\lambda}^{x}\right)=U_{1}^{x / 2}$ for all $x \in U, \lambda \in A$. Clearly $T$ has no fixed point. However it is a soft contraction with soft constant contraction $\bar{c}$ defined as $\bar{c}(\lambda)=1 / 2$ for all $\lambda \in A$. Indeed, fix $x, y \in U$ and $\lambda, \mu \in A$, then for each $\eta \in A$ we have

$$
\begin{aligned}
d\left(T\left(U_{\lambda}^{x}\right), T\left(U_{\mu}^{y}\right)\right)(\eta) & =d\left(U_{1}^{x / 2}, U_{1}^{y / 2}\right)(\eta)=\left|\frac{x}{2}-\frac{y}{2}\right| \\
& \leq \frac{1}{2}(|x-y|+|\lambda-\mu|)=\bar{c}\left(d\left(T\left(U_{\lambda}^{x}\right), T\left(U_{\mu}^{y}\right)\right)\right)(\eta) .
\end{aligned}
$$

Hence $d\left(T\left(U_{\lambda}^{x}\right), T\left(U_{\mu}^{y}\right)\right) \tilde{\leq} \bar{c} d\left(T\left(U_{\lambda}^{x}\right), T\left(U_{\mu}^{y}\right)\right)$.

Remark 4.2.23 We have discovered that Murat I. Yazar, Cigdem Gunduz (Aras) and Sadi Bayramov have established in Theorem 4.8 of their paper 
"Fixed point theorems of soft contractive mappings" [135], a similar result to our main theorem (Theorem 4.2.16) but without assuming that the set $A$ is finite. Our Example 4.2.22 shows that their result is not correct (the error seems occur on line -3 of page 9 ). Furthermore, if in Example 4.2.22 we put $x / 3$ instead of $x / 2$, a counterexample to Theorem 4.9 of Yazar-GunduzBayramov's paper, is also obtained. 


\section{Chapter 5}

\section{Multivalued Caristi's Type Mappings in Fuzzy Metric Spaces and a Characterization of Fuzzy Metric Completeness}

The material of this chapter is an adaptation to the thesis of the content of the paper by Muhajid Abbas, Basit Ali and Salvador Romaguera, "Multivalued Caristi's type mappings in fuzzy metric spaces and a characterization of fuzzy metric completeness", which is accepted for publication in the JCR-journal FILOMAT 


\subsection{Introduction and preliminaries}

Since the appearance of celebrated Banach contraction principle in 1932, several generalizations of this theorem in the setting of point to point mappings have been obtained. Caristi fixed point theorem is one of the most important extension of Banach contraction principle. It is known that this theorem is equivalent to Ekland variational principle [36] , an important tool in nonlinear analysis.

In the year 1969, Nadler combined the ideas of multivalued mappings and contractions proving a generalization of Banach contraction principle. He proved that any multivalued contractive mapping of a complete metric space $X$ into the family of closed and bounded subsets of $X$ has a fixed point.

The mathematical modelling of fuzzy concepts was initiated by Zadeh [136] in 1965. With the advancement of automatic control and expert systems, an involvement of fuzzy concepts in technology has increased rapidly. The study of fuzzy topology, an important branch of fuzzy theory has received much attention for the last two decades. The concept of a fuzzy metric spaces is one of the central theme of Fuzzy topology. Many authors defined and generalized the concept of a fuzzy metric space ([31], [74]). George and Veeramani $([46,47])$ modified the concept of a fuzzy metric space introduced by Kramosil and Michalek [80] and showed that every metric induces a fuzzy metric.

In this chapter, we obtain a fixed point theorem for multivalued mappings of Caristi's type in complete fuzzy metric spaces which actually provides a characterization of fuzzy metric completeness in the case of continuous tnorms greater than or equal to the Lukasiewicz t-norm. We recall that previous and interesting versions of Caristi's fixed point theorem for fuzzy metric spaces (actually, for probabilistic Menger spaces), but with an approach different from our one, were proved by Hadžić and Pap ([53, Section 3.4]). Thus the results presented in this chapter and the ones given in [53, Section 3.4] 
are of an independent value.

First, we recall some pertinent concepts and results.

Following [117], a binary operation $*:[0,1]^{2} \rightarrow[0,1]$ is called a continuous t-norm if: (i) $*$ is associative and commutative; (ii) $*$ is continuous; (iii) $a * 1=a$ for all $a \in[0,1]$; and (iv) $a * b \leq c * d$ whenever $a \leq c$ and $b \leq d$.

Typical instances of continuous t-norm are $\wedge$, $\cdot$ and $*_{L}$, where, for all $a, b \in[0,1], a \wedge b=\min \{a, b\}, a \cdot b=a b$, and $*_{L}$ is the Lukasiewicz t-norm defined by $a *_{L} b=\max \{a+b-1,0\}$.

It is easy to check that $*_{L} \leq \cdot \leq \wedge$. In fact $* \leq \wedge$ for all continuous t-norm $*$.

In our context we will use the following notion of a fuzzy metric space which is a slight modification to the one given by Kramosil and Michalek in [80] (it is appropriate to point out that George and Veeramani presented in $[46,47]$ a stronger but interesting notion of fuzzy metric completeness, which will not be explicitly considered here).

Definition 5.1.1 (compare [80]) A fuzzy metric space is a triple $(X, M, *)$ such that $*$ is a continuous t-norm and $M$ is a fuzzy set in $X \times X \times[0,+\infty)$ such that for all $x, y, z \in X$ :

(i) $M(x, y, 0)=0$;

(ii) $x=y$ if and only if $M(x, y, t)=1$ for all $t>0$;

(iii) $M(x, y, t)=M(y, x, t)$;

(iv) $M(x, z, t+s) \geq M(x, y, t) * M(y, z, s)$ for all $t, s \geq 0$;

(v) $M\left(x, y,_{-}\right):[0,+\infty) \rightarrow[0,1]$ is left continuous.

In this case, the pair $(M, *)$ (or simply, $M$ if no confusion arises) is said to be a fuzzy metric on $X$.

It is well known, and easy to see, that for each $x, y \in X, M\left(x, y,{ }_{-}\right)$is a non-decreasing function on $[0,+\infty)$.

Each fuzzy metric $(M, *)$ on a set $X$ induces a topology $\tau_{M}$ on $X$ which has a base the family of open balls $\left\{B_{M}(x, \varepsilon, t): x \in X, \varepsilon \in(0,1), t>0\right\}$, 
where $B_{M}(x, \varepsilon, t)=\{y \in X: M(x, y, t)>1-\varepsilon\}$.

Observe that a sequence $\left(x_{n}\right)_{n \in \mathbb{N}}$ converges to $x \in X$ (with respect to $\tau_{M}$ ) if and only if $\lim _{n \rightarrow \infty} M\left(x, x_{n}, t\right)=1$ for all $t>0$.

It is also well known (see, for instance, [50]) that every fuzzy metric space $(X, M, *)$ is metrizable, i.e., there exists a metric $d$ on $X$ whose induced topology agrees with $\tau_{M}$.

Conversely, if $(X, d)$ is a metric space and we define $M_{d}: X \times X \times$ $[0,+\infty) \rightarrow[0,1]$ by $M(x, y, 0)=0$ and

$$
M_{d}(x, y, t)=\frac{t}{t+d(x, y)},
$$

for all $t>0$, then $\left(X, M_{d}, \wedge\right)$ is a fuzzy metric space and $\left(M_{d}, \wedge\right)$ is called the standard fuzzy metric of $(X, d)([46])$. Moreover, the topology $\tau_{M_{d}}$ agrees with the topology induced by $d$.

A sequence $\left(x_{n}\right)_{n \in \mathbb{N}}$ in a fuzzy metric space $(X, M, *)$ is said to be a Cauchy sequence if for each $t>0$ and $\varepsilon \in(0,1)$ there exists $n_{0} \in \mathbb{N}$ such that $M\left(x_{n}, x_{m}, t\right)>1-\varepsilon$ for all $n, m \geq n_{0}$.

A fuzzy metric space $(X, M, *)$ is said to be complete ([47]) if every Cauchy sequence converges.

\subsection{The results}

Let $(X, d)$ be a metric space. A mapping $T: X \rightarrow X$ is called a Caristi's mapping if there is a lower semicontinuous function $\varphi: X \rightarrow[0,+\infty)$ satisfying the following condition

$$
d(x, T x) \leq \varphi(x)-\varphi(T x),
$$

for all $x \in X$.

Caristi proved in [24] his celebrated theorem that every Caristi's mapping on a complete metric space has a fixed point. 
Later on, Kirk proved the following nice characterization of metric completeness.

Theorem 5.2.1 [78] A metric space $(X, d)$ is complete if and only if every Caristi's mapping $T: X \rightarrow X$ has a fixed point.

In the sequel, we shall denote by $\mathcal{C}_{0}(X)$ the set of all non-empty closed subsets of a metric space $(X, d)$, or of a fuzzy metric space $(X, M, *)$.

There exist several multivalued generalizations of Caristi's fixed point theorem in the literature. For our purposes here we need the following.

Theorem 5.2.2 (see e.g. [14]) Let $(X, d)$ be a complete metric space and let $T: X \rightarrow \mathcal{C}_{0}(X)$ be a multivalued mapping such that there is a lower semicontinuous function $\varphi: X \rightarrow[0,+\infty)$ satisfying the following condition: For each $x \in X$ there is $y \in T x$ with

$$
d(x, y) \leq \varphi(x)-\varphi(y)
$$

Then $T$ has a fixed point, i.e., there is $z \in X$ such that $z \in T z$.

A multivalued mapping $T$ satisfying the conditions of the preceding theorem will be called a Caristi's multivalued mapping (for $(X, d)$ ).

Definition 5.2.3 Let $(X, M, *)$ be a fuzzy metric space. We say that $T$ : $X \rightarrow X$ is a fuzzy Caristi's mapping on $X$ if there is a lower semicontinuous function $\varphi: X \rightarrow[0,+\infty)$ satisfying the following condition:

$\left(\mathrm{I}_{C}\right) \quad \varphi(x)-\varphi(T x)<t \Longrightarrow M(x, T x, t)>1-t$.

The next example shows that every Caristi's mapping on a metric space $(X, d)$ is a Caristi's mapping on a well-known fuzzy metric space induced by $(X, d)$ in a natural way.

Example 5.2.4 Let $(X, d)$ be a metric space and let $T: X \rightarrow X$ be a Caristi's mapping. Consider the fuzzy metric $(M, *)$ on $X$ (where $*$ is any continuous t-norm) given by $M(x, y, t)=1$ if $d(x, y)<t$, and $M(x, y, t)=0$ 
if $d(x, y) \geq t$ (with $t \geq 0$ ). We show that $T$ is a fuzzy Caristi's mapping for $(X, M, *)$. Indeed, let $\varphi: X \rightarrow[0,+\infty)$ be a lower semicontinuous function for $(X, d)$ such that $d(x, T x) \leq \varphi(x)-\varphi(T x)$, for all $x \in X$. First observe that $\varphi$ is also lower semicontinuous for $(X, M, *)$ because the topology $\tau_{M}$ agrees with the topology induced by $d$. Now suppose that $\varphi(x)-\varphi(T x)<t$ for any $t>0$. Then $d(x, T x)<t$, so $M(x, T x, t)=1$. Hence, condition $\left(\mathbf{I}_{C}\right)$ is satisfied and thus $T$ is a fuzzy Caristi's mapping for $(X, M, *)$.

Definition 5.2.5 Let $(X, M, *)$ be a fuzzy metric space. We say that $T: X \rightarrow \mathcal{C}_{0}(X)$ is a fuzzy Caristi's multivalued mapping on $X$ if there is a lower semicontinuous function $\varphi: X \rightarrow[0,+\infty)$ satisfying the following condition:

$\left(\mathrm{I}_{C M}\right) \quad$ For each $x \in X$ there is $y_{x} \in T x$ such that

$$
\varphi(x)-\varphi\left(y_{x}\right)<t \Longrightarrow M\left(x, y_{x}, t\right)>1-t \text {. }
$$

It is clear that every fuzzy Caristi's mapping can be considered as a fuzzy Caristi's multivalued mapping.

Consider now a fuzzy metric space $(X, M, *)$ with $* \geq *_{L}$. Then, Radu proved in [105] (see also [25, Remark 7.6.1]) that the function $d_{M}$ defined on $X \times X$ by

$$
d_{M}(x, y)=\sup \{t \geq 0: M(x, y, t) \leq 1-t\}
$$

for all $x, y \in X$, is a metric on $X$. Moreover, is is easy to check that for each $x, y \in X$ and $t \in(0,1)$, we have

$$
d_{M}(x, y)<t \Longleftrightarrow M(x, y, t)>1-t .
$$

From this fact it is easily deduced the following.

Proposition 5.2.6 Let $(X, M, *)$ be a fuzzy metric space such that $* \geq *_{L}$. Then $(X, M, *)$ is complete if and only if the metric space $\left(X, d_{M}\right)$ is complete.

With the help of the above results and facts we can prove the following. 
Theorem 5.2.7 Let $(X, M, *)$ be a fuzzy metric space such that $* \geq *_{L}$. Then, the following are equivalent:

(1) $(X, M, *)$ is complete.

(2) Every fuzzy Caristi's multivalued mapping $T: X \rightarrow \mathcal{C}_{0}(X)$ has a fixed point.

(3) Every fuzzy Caristi's mapping $T: X \rightarrow X$ has a fixed point.

Proof. $\quad(1) \Rightarrow(2)$. Since $(X, M, *)$ is complete, then the metric space $\left(X, d_{M}\right)$ is complete by Proposition 5.2.6.

Let $T: X \rightarrow \mathcal{C}_{0}(X)$ be a fuzzy Caristi's multivalued mapping. Then, there exists a lower semicontinuous function $\varphi: X \rightarrow[0,+\infty)$ for which condition $\left(\mathrm{I}_{C M}\right)$ holds.

Let $x \in X$. By $\left(\mathrm{I}_{C M}\right)$, there is $y_{x} \in T x$ such that

$$
\varphi(x)-\varphi\left(y_{x}\right)<t \Longrightarrow M\left(x, y_{x}, t\right)>1-t .
$$

Suppose $\varphi(x)-\varphi\left(y_{x}\right)<d_{M}\left(x, y_{x}\right)$. Then, there exists $t_{0}>0$ such that $\varphi(x)-\varphi\left(y_{x}\right)<t_{0} \leq 1-M\left(x, y_{x}, t_{0}\right)$, so $M\left(x, y_{x}, t_{0}\right) \leq 1-t_{0}$, which contradicts condition $\left(\mathrm{I}_{C M}\right)$. Therefore, $d_{M}\left(x, y_{x}\right) \leq \varphi(x)-\varphi\left(y_{x}\right)$. Hence, we can apply Theorem 5.2.2 and thus $T$ has a fixed point.

$(2) \Rightarrow(3)$. Obvious.

$(3) \Rightarrow(1)$. Let $T: X \rightarrow X$ be a Caristi's mapping for the metric space $\left(X, d_{M}\right)$. We shall prove that $T$ has a fixed point. Indeed, there exists a lower semicontinuous function $\varphi: X \rightarrow[0,+\infty)$ such that

$$
d_{M}(x, T x) \leq \varphi(x)-\varphi(T x)
$$

for all $x \in X$.

Suppose $\varphi(x)-\varphi(T x)<t$. Then $d_{M}(x, T x)<t$, and hence $M(x, T x, t)>$ $1-t$. We have shown that condition $\left(\mathrm{I}_{C}\right)$ is satisfied, so $T$ is a fuzzy Caristi's mapping for $(X, M, *)$. By our hypothesis $T$ has a fixed point. Therefore $\left(X, d_{M}\right)$ is complete by Theorem 5.2.1. We conclude that $(X, M, *)$ is complete by Proposition 5.2.6. 
The following natural question remains open:

Question. Is it possible to generalize Theorem 5.2.6 to the case that $*$ is any continuous t-norm?

We conclude the chapter with two examples that illustrate Theorem 5.2.6.

Example 5.2.8 Let $X=(0,1]$ and let $(M, *)$ be the fuzzy metric on $X$, with $* \geq *_{L}$, given by $M(x, y, 0)=0$ for all $x, y \in X, M(x, x, t)=1$ for all $x \in X$ and $t>0$, and $M(x, y, t)=x * y$ otherwise.

First note that $x=1$ is the unique non-isolated point of $X$, because

$$
B_{M}(1,1 / n, 1 / n)=\{y \in X: y>1-1 / n\}
$$

for all $n \in \mathbb{N}$, while for each $x \in X \backslash\{1\}$, one has

$$
B_{M}(x, x, 1-x)=\{x\} \cup\{y \in X: x * y>x\}=\{x\}
$$

Consequently $(X, M, *)$ is complete. In fact, if $\left(x_{n}\right)_{n \in \mathbb{N}}$ is a non-eventually constant Cauchy sequence, then for each $\varepsilon \in(0,1)$ there is $n_{0} \in \mathbb{N}$ such that $x_{n} * x_{m}>1-\varepsilon$ for all $n, m \geq n_{0}$, so $\left(x_{n}\right)_{n \in \mathbb{N}}$ converges to 1 with respect to the Euclidean topology and hence with respect to $\tau_{M}$.

Define $T: X \rightarrow \mathcal{C}_{0}(X)$ by $T x=[\sqrt{x}, 1]$. We show that $T$ is a multivalued Caristi's fuzzy mapping on $(X, M, *)$. Indeed, let $\varphi: X \rightarrow[0,+\infty)$ be the lower semicontinuous function on $X$ defined by

$$
\varphi(1)=0 \text { and } \varphi(x)=1 \text { otherwise. }
$$

Let $x \in X$. Take $y_{x}=1 \in T x$, and suppose $\varphi(x)-\varphi\left(y_{x}\right)<t$. Then $t>1$, and consequently $M\left(x, y_{x}, t\right) \geq 0>1-t$. We have shown that condition $\left(\mathbf{I}_{C M}\right)$ is satisfied, and hence $T$ is a multivalued Caristi's fuzzy mapping. Clearly 1 is a fixed point of $T$ (in fact, it is the only fixed point of $T$ ). 
Example 5.2.9 Let $X=(0,1]$ and let $(M, \wedge)$ be the fuzzy metric on $X$ given by $M(x, y, 0)=0$ for all $x, y \in X, M(x, x, t)=1$ for all $x \in X$ and $t>0$, and $M(x, y, t)=\min \{x, y, t\}$ otherwise.

First note that $\tau_{M}$ is the discrete topology on $X$ because for each $x \in X$ one has

$$
B_{M}(x, 1 / 2,1 / 2)=\{x\} \cup\{y \in X: \min \{x, y, 1 / 2\}>1 / 2\}=\{x\}
$$

Moreover $(X, M, *)$ is complete because for $x \neq y$ and $t \in(0,1)$ we have $M(x, y, t) \leq t$ and hence the eventually constant sequences are the only Cauchy sequences in $(X, M, \wedge)$.

Denote by $\mathbb{Q}$ the set of rational numbers and define $T: X \rightarrow \mathcal{C}_{0}(X)$ by

$$
T x=\left\{y \in(0,1] \cap \mathbb{Q}: x \leq y \leq \frac{x+1}{2}\right\},
$$

for all $x \in X$. Note that, indeed, $T x \in \mathcal{C}_{0}(X)$ since $\tau_{M}$ is the discrete topology on $X$.

We show that $T$ is a multivalued Caristi's fuzzy mapping on $(X, M, *)$. Indeed, let $\varphi: X \rightarrow[0,+\infty)$ be the lower semicontinuous function on $X$ defined by $\varphi(x)=0$ if $x \in(0,1] \cap \mathbb{Q}$ and $\varphi(x)=1$ otherwise. Let $x \in X$. If $x \in \mathbb{Q}$ take $y_{x}=x \in T x$. Then $M\left(x, y_{x}, t\right)=1$ for all $t>0$, so condition $\left(\mathbf{I}_{C M}\right)$ is trivially satisfied in this case. If $x \notin \mathbb{Q}$, take $y_{x}$ a rational number belonging to $(x, x+1 / 2)$ and suppose $\varphi(x)-\varphi\left(y_{x}\right)<t$. Then $t>1$, and consequently $M\left(x, y_{x}, t\right) \geq 0>1-t$. Therefore $T$ is a multivalued Caristi's fuzzy mapping. Clearly $x \in T x$ for all $x \in(0,1] \cap \mathbb{Q}$ (in particular $T 1=\{1\}$ ). 


\section{Chapter 6}

\section{Fixed and Periodic Points of Generalized Contractions in Metric Spaces and in Normed Spaces}

The material of Sections 6.2 and 6.3 of this chapter is an adaptation to the thesis of the content of the paper by Muhajid Abbas, Basit Ali and Salvador Romaguera, "Fixed and periodic points of generalized contractions in metric spaces", published in the JCR-journal Fixed Point Theory and Applications 2013, 2013:243. The material of Sections 6.4 and 6.5 is an adaptation to the thesis of the content of the paper by Muhajid Abbas, Basit Ali and Salvador Romaguera, "Generalized contraction and invariant approximation results on nonconvex subsets of normed spaces", published in the JCR-journal Abstract and Applied Analysis 2014 (2014), Article ID 391952. 


\subsection{Introduction and preliminaries}

Fixed point theory can mainly be classified into three different areas (a) Topological fixed point theory (b) Order oriented fixed point theory (c) Metric fixed point theory, each is determined by underlying mathematical structure. Topological fixed point theory is based on Brouwer's fixed point theorem. It is worth mentioning that Brouwer's theorem is not constructive and does not give information about how to find the fixed point of a given mapping. Order oriented fixed point theory is studied in the environment created by the class of partially ordered sets along with appropriate mappings satisfying certain conditions like monotonicity, expansivity, or various forms of order continuity. Zermelo's theorem, the famous Tarski fixed point theorem, Fujimoto fixed point theorem provide the basis for order theoretic fixed point theory. These theorems are existential in nature. Metric fixed point theory is studied in the framework of a set endowed with some notion of a distance and is based on Banach contraction principle. Contrary to Brouwer's fixed point theorem, Banach contraction principle not only solves the problem on the existence of a unique solution to an operator equation but also gives a practical method to obtain the approximation of a solution. Being based on an iteration process, it can be implemented on a computer to find the fixed point of a contractive mapping.

Recently, Wardowski [129] introduced a new type of contraction called $F$-contraction and proved a fixed point result in complete metric spaces which in turn generalizes the Banach contraction principle. In this chapter, we introduce $F$-contractions with respect to a self mapping on a metric space and obtain common fixed point results in a partially ordered sets equipped with a complete metric. From this perspective, our results can be viewed as a

mixture of metric fixed point and order oriented fixed point results. We also introduced a notion of generalized $F$ - contraction and employed this concept to prove a fixed point theorem for generalized nonexpansive mapping. As an 
application of our results, periodic point results for the $F$-contractions in metric spaces are also proved. Examples are provided to support results and concepts presented herein.

We first present some definitions and known results needed in the sequel. Definition 6.1.1 Let $f$ and $g$ be self mappings on a set $X$. If $f x=g x=w$ for some $x$ in $X$, then $x$ is called a coincidence point of $f$ and $g$ and $w$ is called a point of coincidence of $f$ and $g$. Furthermore, if $f g x=g f x$ whenever $x$ is a coincidence point of $f$ and $g$, then $f$ and $g$ are called weakly compatible mappings [67].

Let $C(f, g)=\{x \in X: f x=g x\}(F(f, g)=\{x \in X: x=f x=g x\})$ denote the set of all coincidence points (the set of all common fixed points) of self mappings $f$ and $g$.

Definition 6.1.2 [70] Let $(X, d)$ be a metric space and $f, g: X \rightarrow X$. The mapping $f$ is called a $g$-contraction if there exists $\alpha \in(0,1)$ such that

$$
d(f x, f y) \leq \alpha d(g x, g y)
$$

holds for all $x, y \in X$.

In 1976, Jungck [70] obtained the following useful generalization of Banach contraction principle.

Theorem 6.1.3 Let $g$ be a continuous self mapping on a complete metric space $(X, d)$. Then $g$ has a fixed point in $X$ if and only if there exists a $g$-contraction mapping $f: X \rightarrow X$ such that $f$ commutes with $g$ and $g(X) \subseteq f(X)$.

In the sequel by $\mathbb{R}_{+}$we shall denote the set of positive real numbers.

Let $\digamma$ be the collection of all mappings $F: \mathbb{R}_{+} \rightarrow \mathbb{R}$ that satisfy the following conditions:

(C1) $F$ is strictly increasing, that is for all $\alpha, \beta \in \mathbb{R}_{+}$such that $\alpha<\beta$ implies that $F(\alpha)<F(\beta)$. 
(C2) For every sequence $\left\{\alpha_{n}\right\}_{n \in \mathbb{N}}$ of positive real numbers, $\lim _{n \rightarrow \infty} \alpha_{n}=0$ and $\lim _{n \rightarrow \infty} F\left(\alpha_{n}\right)=-\infty$ are equivalent.

(C3) There exists $k \in(0,1)$ such that

$$
\lim _{\alpha \rightarrow 0^{+}} \alpha^{k} F(\alpha)=0
$$

Definition 6.1.4 [129] Let $(X, d)$ be a metric space. A mapping $f: X \rightarrow X$ is said to be an $F$-contraction on $X$ if there exists $\tau>0$ such that

$$
d(f x, f y)>0 \text { implies that } \tau+F(d(f x, f y)) \leq F(d(x, y))
$$

for all $x, y \in X$, and $F \in \digamma$.

Note that every $F$-contraction is continuous (see [129]). We extend above definition to two mappings.

Definition 6.1.5 Let $(X, d)$ be a metric space and $f, g: X \rightarrow X$. The mapping $f$ is said to be an $F$-contraction with respect to $g$ on $X$, if there exists $\tau>0$ such that

$$
\tau+F(d(f x, f y)) \leq F(d(g x, g y))
$$

for all $x, y \in X$ satisfying $\min \{d(f x, f y), d(g x, g y)\}>0$, and $F \in \digamma$.

By different choices of mappings $F$ in (6.1.1) and (6.1.2), one obtains a variety of contractions $([129])$.

Remark 6.1.6 Let $F_{1}: \mathbb{R}_{+} \rightarrow \mathbb{R}$ be given by $F_{1}(\alpha)=\ln (\alpha)$. It is clear that $F \in \digamma$. Suppose that $f: X \rightarrow X$ is an $F$-contraction with respect to a self mapping $g$ on $X$. From (6.1.2), we have

$$
\tau+\ln (d(f x, f y)) \leq \ln (d(g x, g y))
$$

which implies that

$$
d(f x, f y) \leq e^{-\tau} d(g x, g y)
$$

Therefore $F_{1}$-contraction map $f$ with respect to $g$ reduces to a $g$-contraction mapping. 
Now we give an example of an $F$-contraction with respect to a self mapping $g$ on $X$ which is not a $g$-contraction on $X$.

Example 6.1.7 Consider the following sequence of partial sums $\left\{S_{n}\right\}_{n \in \mathbb{N}}$ [129, Example 2.5]

$$
\begin{aligned}
S_{1}= & 1, \\
S_{2}= & 1+2 \\
S_{3}= & 1+2+3 \\
& \ldots \\
S_{n}= & 1+2+\ldots+n=\frac{n(n+1)}{2}, n \in \mathbb{N} .
\end{aligned}
$$

Let $X=\left\{S_{n}: n \in \mathbb{N}\right\}$ and $d$ be the usual metric on $X$. Let $f: X \rightarrow X$ and $g: X \rightarrow X$ be defined as:

$$
f S_{n}=\left\{\begin{array}{c}
S_{n-1}, \text { if } n>1, \\
S_{1}, \text { if } n=1
\end{array}, g S_{n}=\left\{\begin{array}{c}
S_{n+1}, \text { if } n>1 \\
S_{1}, \text { if } n=1
\end{array}\right.\right.
$$

Let $F_{1}: \mathbb{R}_{+} \rightarrow \mathbb{R}$ be given by $F_{1}(\alpha)=\ln (\alpha)$. As

$$
\lim _{n \rightarrow \infty} \frac{d\left(f S_{n}, f S_{1}\right)}{d\left(g S_{n}, g S_{1}\right)}=\lim _{n \rightarrow \infty} \frac{S_{n-1}-S_{1}}{S_{n+1}-S_{1}}=1
$$

so $f$ is not a $g$-contraction. If we take $F_{2}(\alpha)=\ln (\alpha)+\alpha$, then $F_{2} \in \digamma$ and $f$ is $F_{2}$-contraction with respect to mapping $g$ (taking $\tau=2$ ). Indeed, following holds:

$$
\begin{aligned}
& \frac{d\left(f S_{n}, f S_{1}\right)}{d\left(g S_{n}, g S_{1}\right)} e^{d\left(f S_{n}, f S_{1}\right)-d\left(g S_{n}, g S_{1}\right)} \\
= & \frac{S_{n-1}-S_{1}}{S_{n+1}-S_{1}} e^{S_{n-1}-S_{1}-S_{n+1}+S_{1}} \\
= & \frac{n^{2}-n-2}{n^{2}+3 n} e^{-4 n-2} \leq e^{-2},
\end{aligned}
$$


for all $n>1$. For all $m, n \in \mathbb{N}$ with $m>n>1$, we have

$$
\begin{aligned}
& \frac{d\left(f S_{m}, f S_{n}\right)}{d\left(g S_{m}, g S_{n}\right)} e^{d\left(f S_{m}, f S_{n}\right)-d\left(g S_{m}, g S_{n}\right)} \\
= & \frac{S_{m-1}-S_{n-1}}{S_{m+1}-S_{n+1}} e^{S_{m-1}-S_{n-1}-S_{m+1}+S_{n+1}} \\
= & \frac{m^{2}+m-n^{2}-n}{m^{2}+3 m-n^{2}-3 n} e^{-2(m-n)} \leq e^{-2} .
\end{aligned}
$$

Definition 6.1.8 [3] (Dominance Condition) Let $(X, \preceq)$ be a partially ordered set. A self mapping $f$ on $X$ is said to be (i) dominated map if $f x \preceq x$ for each $x$ in $X$, (ii) dominating map if $x \preceq f x$ for each $x$ in $X$.

Example 6.1.9 Let $X=[0,1]$ be endowed with the usual ordering and $f, g: X \rightarrow X$ defined by $g x=x^{n}$ for some $n \in \mathbb{N}$ and $f x=k x$ for some real number $k \geq 1$. Note that

$$
g x=x^{n} \leq x \text { and } x \leq k x=f x
$$

for all $x$ in $X$. Thus $g$ is dominated and $f$ is a dominating map.

Definition 6.1.10 Let $(X, \preceq)$ be a partially ordered set. Two mappings $f, g: X \rightarrow X$ are said to be weakly increasing if $f x \preceq g f x$ and $g x \preceq f g x$ for all $x$ in $X$ (see [11]).

Definition 6.1.11 Let $X$ be a nonempty set. Then $(X, d, \preceq)$ is called an ordered metric space if $(X, d)$ is a metric space and $(X, \preceq)$ is a partially ordered set.

Definition 6.1.12 Let $(X, \leq)$ be an ordered set. A pair $(f, g)$ on $X$ is said:

(i) weakly increasing if for all $x \in X$, we have $f x \leq g f x$ and $g x \leq f g x,[12]$

(ii) partially weakly increasing if $f x \leq g f x$, for all $x \in X$.

Remark 6.1.13 A pair $(f, g)$ is weakly increasing if and only if ordered pair $(f, g)$ and $(g, f)$ are partially weakly increasing.

Example 6.1.14 Let $X=[0,1]$ be endowed with usual ordering. Let $f, g: X \rightarrow X$ be defined by $f x=x^{2}$ and $g x=\sqrt{x}$. Then $f x=x^{2} \leq x=g f x$ for all $x \in X$. Thus $(f, g)$ is partially weakly increasing. But $g x=\sqrt{x} \not \leq x=$ $f g x$ for $x \in(0,1)$. So $(g, f)$ is not partially weakly increasing. 
Definition 6.1.15 Let $(X, \leq)$ be an ordered set. A mapping $f$ is called weak annihilator of $g$ if $f g x \leq x$ for all $x \in X$.

Example 6.1.16 Let $X=[0,1]$ be endowed with usual ordering. Define $f, g: X \rightarrow X$ by $f x=x^{2}$ and $g x=x^{3}$. Then $f g x=x^{6} \leq x$ for all $x \in X$. Thus $f$ is a weak annihilator of $g$.

Definition 6.1.17 Let $(X, \leq)$ be an ordered set. A selfmap $f$ on $X$ is called dominating map if $x \leq f x$ for each $x$ in $X$.

Example 6.1.18 Let $X=[0,1]$ be endowed with usual ordering. Let $f: X \rightarrow X$ be defined by $f x=x^{\frac{1}{3}}$. Then $x \leq x^{\frac{1}{3}}=f x$ for all $x \in X$. Thus $f$ is a dominating map.

Example 6.1.19 Let $X=[0, \infty)$ be endowed with usual ordering. Define $f: X \rightarrow X$ by

$$
f x=\left\{\begin{array}{c}
\sqrt[n]{x} \text { for } x \in[0,1), \\
x^{n} \text { for } x \in[1, \infty),
\end{array}\right.
$$

$n \in N$. Then for all $x \in X, x \leq f x$ so that $f$ is a dominating map.

Definition 6.1.20 Let $(X, \preceq)$ be a partial ordered set, then $x, y$ in $X$ are called comparable elements if either $x \preceq y$ or $y \preceq x$ holds true. Moreover, we define $\Delta \subseteq X \times X$ by

$$
\Delta=\{(x, y) \in X \times X: x \preceq y \text { or } y \preceq x\}
$$

Definition 6.1.21 An ordered metric space $(X, d, \preceq)$ is said to have sequential limit comparison property if for every non-decreasing sequence (nonincreasing sequence) $\left\{x_{n}\right\}_{n \in \mathbb{N}}$ in $X$ such that $x_{n} \rightarrow x$ implies that $x_{n} \preceq x$ $\left(x \preceq x_{n}\right)$.

\subsection{Common fixed point results in ordered metric spaces}

We present following theorem as a generalization of results in [71] and [129, Theorem 2.1]. 
Theorem 6.2.1 Let $(X, \preceq)$ be a partially ordered set such that there exists a metric $d$ on $X$ and $f: X \rightarrow X$ an $F$-contraction with respect to $g: X \rightarrow X$ on $\Delta$ with $f(X) \subseteq g(X)$. Assume that $f$ is dominating and $g$ is dominated. Then

(a) $f$ and $g$ have a coincidence point in $X$ provided that $g(X)$ is complete and has sequential limit comparison property.

(b) $C(f, g)$ is well ordered if and only if $C(f, g)$ is singleton.

(c) $f$ and $g$ have unique common fixed point if $f$ and $g$ are weakly compatible and $C(f, g)$ is well ordered.

Proof. (a) Let $x_{0}$ be an arbitrary point of $X$. Since the range of $g$ contains the range of $f$, so there exists a point $x_{1}$ in $X$ such that $f\left(x_{0}\right)=g\left(x_{1}\right)$. As $f$ is dominating and $g$ is dominated, so we have

$$
x_{0} \preceq f x_{0}=g x_{1} \preceq x_{1} .
$$

Hence $\left(x_{0}, x_{1}\right) \in \Delta$. Continuing this process, having chosen $x_{n}$ in $X$, we obtain $x_{n+1}$ in $X$ such that

$$
x_{n} \preceq f x_{n}=g x_{n+1} \preceq x_{n+1} .
$$

So we obtain $\left(x_{n}, x_{n+1}\right) \in \Delta$ for every $n \in \mathbb{N} \cup\{0\}$. For sake of simplicity, take

$$
\gamma_{n}=d\left(g x_{n}, g x_{n+1}\right)
$$

for all $n \in \mathbb{N} \cup\{0\}$. If there exists $n_{0} \in \mathbb{N} \cup\{0\}$ for which $x_{n_{0}+1}=x_{n_{0}}$, then $f x_{n_{0}}=g x_{n_{0}+1}$ implies that $f x_{n_{0}+1}=g x_{n_{0}+1}$, that is, $x_{n_{0}+1} \in C(f, g)$. Now we assume that $x_{n+1} \neq x_{n}$ for all $n \in \mathbb{N} \cup\{0\}$. As $f$ is $F$-contraction with 
respect to $g$ on $\Delta$, so we obtain

$$
\begin{aligned}
F\left(\gamma_{n}\right) & =F\left(d\left(g x_{n}, g x_{n+1}\right)\right)=F\left(d\left(f x_{n-1}, f x_{n}\right)\right) \\
& \leq F\left(d\left(g x_{n-1}, g x_{n}\right)\right)-\tau \\
& =F\left(d\left(f x_{n-2}, f x_{n-1}\right)\right)-\tau \\
& \leq F\left(d\left(g x_{n-2}, g x_{n-1}\right)\right)-2 \tau \leq \ldots \\
& \leq F\left(d\left(g x_{1}, g x_{2}\right)\right)-(n-1) \tau=F\left(\gamma_{1}\right)-(n-1) \tau
\end{aligned}
$$

That is

$$
F\left(\gamma_{n}\right) \leq F\left(\gamma_{1}\right)-(n-1) \tau
$$

On taking limit as $n \rightarrow \infty$, we obtain $\lim _{n \rightarrow \infty} F\left(\gamma_{n}\right)=-\infty$. Hence $\lim _{n \rightarrow \infty} \gamma_{n}=0$ by (C2). Now by (C3), there exists $k \in(0,1)$ such that $\lim _{n \rightarrow \infty} \gamma_{n}^{k} F\left(\gamma_{n}\right)=0$. Note that

$$
\gamma_{n}^{k} F\left(\gamma_{n}\right)-\gamma_{n}^{k} F\left(\gamma_{1}\right) \leq \gamma_{n}^{k}\left(F\left(\gamma_{1}\right)-(n-1) \tau\right)-\gamma_{n}^{k} F\left(\gamma_{1}\right)=-\gamma_{n}^{k}(n-1) \tau \leq 0
$$

Taking limit as $n \rightarrow \infty$ in (6.2.2), we have $\lim _{n \rightarrow \infty}(n-1) \gamma_{n}^{k}=0$. Consequently, $\lim _{n \rightarrow \infty} n \gamma_{n}^{k}=0$. Thus there exist $n_{1}$ in $\mathbb{N}$ such that $n \gamma_{n}^{k} \leq 1$ for all $n \geq n_{1}$, that is $\gamma_{n} \leq 1 / n^{1 / k}$ for all $n \geq n_{1}$. Now for integers $m>n \geq 1$, we obtain

$$
\begin{aligned}
d\left(g x_{n}, g x_{m}\right) & \leq d\left(g x_{n}, g x_{n+1}\right)+d\left(g x_{n+1}, g x_{n+2}\right)+\ldots+d\left(g x_{m-1}, g x_{m}\right) \\
& <\sum_{i=n}^{\infty} \gamma_{i} \leq \sum_{i=n}^{\infty} \frac{1}{i^{\frac{1}{k}}}<\infty .
\end{aligned}
$$

This shows that $\left\{g x_{n}\right\}_{n \in \mathbb{N}}$ is a Cauchy sequence in $g(X)$. As $g(X)$ is complete so there exists $q$ in $g(X)$ such that $\lim _{n \rightarrow \infty} g x_{n}=q$. Let $p \in X$ be such that $g(p)=q$. Sequential limit comparison property implies that $g x_{n+1} \preceq q$. As $x_{n} \preceq f x_{n}=g x_{n+1} \preceq q=g(p) \preceq p$ so $\left(x_{n}, p\right) \in \Delta$. Hence from (6.1.2), we have

$$
F\left(d\left(g x_{n}, f p\right)\right)=F\left(d\left(f x_{n-1}, f p\right)\right) \leq F\left(d\left(g x_{n-1}, g p\right)\right)-\tau
$$


Since $\lim _{n \rightarrow \infty} d\left(g x_{n-1}, g p\right)=0$, therefore by $(\mathrm{C} 2)$, we have $\lim _{n \rightarrow \infty} F\left(d\left(g x_{n-1}, g p\right)\right)=$ $-\infty$. Hence $\lim _{n \rightarrow \infty} F\left(d\left(g x_{n}, f p\right)\right)=-\infty$ implies that $\lim _{n \rightarrow \infty} d\left(g x_{n}, f p\right)=0$. That is $\lim _{n \rightarrow \infty} g x_{n}=f p$. Uniqueness of limit implies $f p=g p$, that is, $p \in C(f, g)$.

(b) Now suppose that $C(f, g)$ is well ordered. We prove that $C(f, g)$ is a singleton. Assume on contrary that there exists another point $w$ in $X$ such that $f w=g w$ with $w \neq p$. Since $C(f, g)$ is well ordered so $(w, p) \in \Delta$. Now from (6.1.2), we have

$$
\tau \leq F(d(g w, g p))-F(d(f w, f p))=0
$$

a contradiction. Therefore $w=p$. Hence $f$ and $g$ have a unique coincidence point $p$ in $X$. Converse follows immediately.

(c) Now if $f$ and $g$ are weakly compatible mappings, then we have $f q=$ $f g p=g f p=g q$, that is, $q$ is the coincidence point of $f$ and $g$. But $q$ is the only point of coincidence of $f$ and $g$, so $f q=g q=q$. Hence $q$ is the unique common fixed point of $f$ and $g$.

Example 6.2.2 Let $X=[0,5]$ be endowed with usual metric and usual order. Define mappings $f, g: X \rightarrow X$ by

$$
g x=\left\{\begin{array}{c}
0 \text { if } x \in[0,, 3) \\
3 \text { if } x \in[3,5) \\
5 \text { if } x=5
\end{array}, f x=\left\{\begin{array}{c}
3 \text { if } x \in[0,3) \\
5 \text { if } x \in[3,5]
\end{array}\right.\right.
$$

Clearly $g$ is dominated, and $f$ is dominating. Define $F: \mathbb{R}_{+} \rightarrow \mathbb{R}$ as $F(x)=$ $\ln (x)$. If $x \in[0,3)$ and $y \in[3,5)$ then

$$
\begin{aligned}
F(d(f x, f y)) & =F(d(3,5))=F(2)=\ln (2) \approx 0.693 \\
& <F(d(g x, g y))=F(d(0,3)) \\
& =F(3)=\ln (3) \approx 1.098 .
\end{aligned}
$$

Hence for $\tau \in(0,0.40]$, inequality (6.1.2) is satisfied. Similarly for $x \in[0,3)$ 
and $y=5$, we have

$$
\begin{aligned}
F(d(f x, f y)) & =F(d(3,5))=F(2)=\ln (2) \approx 0.693 \\
& <F(d(g x, g y))=F(d(0,5)) \\
& =F(5)=\ln (5) \approx 1.6094 .
\end{aligned}
$$

Hence for $\tau \in(0,0.9164]$, inequality (6.1.2) is satisfied. We can take a $\tau \in$ $(0,0.40]$ so that

$$
\tau+F(d(f x, f y)) \leq F(d(g x, g y))
$$

is satisfied for all $x, y \in[0,5]$, whenever $\min \{d(f x, f y), d(g x, g y)\}>0$. Hence $f$ is an $F$-contraction with respect to $g$ on $[0,5]$. Hence all the conditions of Theorem 6.2.1 are satisfied. Moreover $x=5$ is the coincidence point of $f$ and $g$. Also note that $f$ and $g$ are weakly compatible and $x=5$ is the common fixed point of $g$ and $f$ as well.

Now we give a common fixed point result without imposing any type of commutativity condition for self mappings $f$ and $g$ on $X$. Moreover we relax the dominance conditions on $f$ and $g$ as well.

Theorem 6.2.3 Let $(X, \preceq)$ be a partially ordered set such that there exists a complete metric $d$ on $X$. If self mappings $f$ and $g$ on $X$ are weakly increasing and for some $\tau>0$ satisfy

$$
\tau+F(d(f x, g y)) \leq F(d(x, y))
$$

for all $(x, y) \in \Delta$ such that $\min \{d(f x, g y), d(x, y)\}>0$. Then $F(f, g) \neq \emptyset$, provided that $X$ has sequential limit comparison property. Further $f$ and $g$ have a unique common fixed point if and only if $F(f, g)$ is well ordered.

Proof. Let $x_{0}$ be an arbitrary point of $X$. Define a sequence $\left\{x_{n}\right\}_{n \in \mathbb{N}}$ in $X$ as follows: $x_{2 n+1}=f x_{2 n}$ and $x_{2 n+2}=g x_{2 n+1}$. Since $f$ and $g$ are weakly increasing so we have $x_{2 n+1}=f x_{2 n} \preceq g f x_{2 n}=g x_{2 n+1}=x_{2 n+2}$ and $x_{2 n+2}=g x_{2 n+1} \preceq f g x_{2 n+1}=f x_{2 n+2}=x_{2 n+3}$. Hence $\left(x_{2 n+1}, x_{2 n+2}\right) \in \Delta$ and $\left(x_{2 n+2}, x_{2 n+3}\right) \in \Delta$ for every $n \in \mathbb{N} \cup\{0\}$. Now denote

$$
\gamma_{2 n}=d\left(x_{2 n+1}, x_{2 n+2}\right)
$$


for all $n \in \mathbb{N} \cup\{0\}$. Using (6.2.3) the following holds for every $n \in \mathbb{N} \cup\{0\}$

$$
\begin{aligned}
F\left(\gamma_{2 n}\right) & =F\left(d\left(x_{2 n+1}, x_{2 n+2}\right)\right)=F\left(d\left(f x_{2 n}, g x_{2 n+1}\right)\right) \\
& \leq F\left(d\left(x_{2 n}, x_{2 n+1}\right)\right)-\tau=F\left(\gamma_{2 n-1}\right)-\tau .
\end{aligned}
$$

Similarly

$$
\begin{aligned}
F\left(\gamma_{2 n+1}\right) & =F\left(d\left(x_{2 n+3}, x_{2 n+2}\right)\right)=F\left(d\left(f x_{2 n+2}, g x_{2 n+1}\right)\right) \\
& \leq F\left(d\left(x_{2 n+1}, x_{2 n+2}\right)\right)-\tau=F\left(\gamma_{2 n}\right)-\tau
\end{aligned}
$$

Therefore, for all $n \in \mathbb{N} \cup\{0\}$, we have

$$
\begin{aligned}
F\left(\gamma_{n}\right) & \leq F\left(\gamma_{n-1}\right)-\tau \leq F\left(\gamma_{n-2}\right)-2 \tau \ldots \\
& \leq F\left(d\left(x_{1}, x_{2}\right)\right)-n \tau=F\left(\gamma_{0}\right)-n \tau
\end{aligned}
$$

Thus

$$
F\left(\gamma_{n}\right) \leq F\left(\gamma_{0}\right)-n \tau
$$

Taking limit as $n \rightarrow \infty$ in (6.2.5) we get

$$
\lim _{n \rightarrow \infty} F\left(\gamma_{n}\right)=-\infty
$$

By (C2) and (C3) we get $\lim _{n \rightarrow \infty} \gamma_{n}=0$ and $k \in(0,1)$ such that $\lim _{n \rightarrow \infty} \gamma_{n}^{k} F\left(\gamma_{n}\right)=$ 0 . Note that

$$
\gamma_{n}^{k} F\left(\gamma_{n}\right)-\gamma_{n}^{k} F\left(\gamma_{0}\right) \leq \gamma_{n}^{k}\left(F\left(\gamma_{0}\right)-n \tau\right)-\gamma_{n}^{k} F\left(\gamma_{0}\right)=-\gamma_{n}^{k} n \tau \leq 0
$$

By taking limit as $n \rightarrow \infty$ in (6.2.6), we get $\lim _{n \rightarrow \infty} n \gamma_{n}^{k}=0$. This implies there exist $n_{1}$ such that $n \gamma_{n}^{k} \leq 1$ for all $n \geq n_{1}$. Consequently we obtain $\gamma_{n} \leq 1 / n^{1 / k}$ for all $n \geq n_{1}$. Now for integers $m>n \geq 1$, we have

$$
\begin{aligned}
d\left(x_{n}, x_{m}\right) & \leq d\left(x_{n}, x_{n+1}\right)+d\left(x_{n+1}, x_{n+2}\right)+\ldots+d\left(x_{m-1}, x_{m}\right) \\
& <\sum_{i=n}^{\infty} \gamma_{i} \leq \sum_{i=n}^{\infty} \frac{1}{i^{\frac{1}{k}}}<\infty
\end{aligned}
$$


This shows that $x_{n}$ is a Cauchy sequence in $X$, so there exist $p$ in $X$ such that $\lim _{n \rightarrow \infty} x_{n}=p$. As $X$ has sequential limit comparison property, so $\left(x_{n}, p\right),\left(x_{2 n}, p\right),\left(x_{2 n+1}, p\right) \in \Delta$. Therefore

$$
\lim _{n \rightarrow \infty} F\left(d\left(x_{2 n+1}, g p\right)\right)=\lim _{n \rightarrow \infty} F\left(d\left(f x_{2 n}, g p\right)\right) \leq F\left(d\left(x_{2 n}, p\right)\right)-\tau .
$$

Since $\lim _{n \rightarrow \infty} d\left(x_{2 n}, p\right)=0$. So by $(\mathrm{C} 2)$, we have $\lim _{n \rightarrow \infty} F\left(d\left(x_{2 n}, p\right)\right)=-\infty$. This implies $\lim _{n \rightarrow \infty} F\left(d\left(x_{2 n+1}, g p\right)\right)=-\infty$ which further implies that $\lim _{n \rightarrow \infty} d\left(x_{2 n+1}, g p\right)=$ 0 . Hence $d(p, g p)=0$ and $p=g p$. Similarly, we obtain $p=f p$. This shows that $p$ is a common fixed point of $g$ and $f$. Now suppose that $F(f, g)$ is well ordered. We prove that $F(f, g)$ is singleton. Assume on contrary that there exists another point $q$ in $X$ such that $q=f q=g q$ with $q \neq p$. Obviously $(q, p) \in \Delta$. So from (6.2.3) we have $\tau \leq F(d(q, p))-F(d(f q, g p))=0$, a contradiction. Therefore $q=p$. Hence $g$ and $f$ have a unique common fixed point $p$ in $X$. Converse follows immediately.

\subsection{Periodic points results in metric spaces}

If $x$ is the fixed point of $f$ then $x$ is the fixed point of $f^{n}$ for every $n \in \mathbb{N}$, but the converse is not true.

Example 6.3.1 Let $f:[0,1] \rightarrow[0,1]$ be given by

$$
f(x)=1-x
$$

Then $f$ has a unique fixed point $x=\frac{1}{2}$. Note that $f^{n} x=x$ holds for every even natural number $n$ and $x$ in $[0,1]$. On the other hand, define a mapping $g:[0, \pi] \rightarrow[0, \pi]$ as

$$
g(x)=\cos x .
$$

Then $g$ has the same fixed point as $g^{n}$ for every $n$.

Definition 6.3.2 The self mapping $f$ is said to have the property $P$ if $F\left(f^{n}\right)=F(f)$ for every $n \in \mathbb{N}$. A pair $(f, g)$ of self mappings is said to have the property $Q$ if $F(f) \cap F(g)=F\left(f^{n}\right) \cap F\left(g^{n}\right)$. 
For further details on these properties, we refer to $[19,64]$.

Let $(X, d)$ be a metric space and $f: X \rightarrow X$ be a self mapping. The set $O(x)=\left\{x, f x, \ldots, f^{n} x, \ldots\right\}$ is called orbit of $x$ [58]. A mapping $f$ is called orbitally continuous at $p$ if $\lim _{n \rightarrow \infty} f^{n} x=p$ implies that $\lim _{n \rightarrow \infty} f^{n+1} x=f p$. A mapping $f$ is orbitally continuous on $X$ if $f$ is orbitally continuous for all $x \in X$.

In this section we prove some periodic points results for self mappings on complete metric spaces.

Theorem 6.3.3 Let $X$ be a nonempty set such that there exists a complete metric $d$ on $X$. Suppose that $f: X \rightarrow X$ satisfies

$$
\tau+F\left(d\left(f x, f^{2} x\right)\right) \leq F(d(x, f x))
$$

for some $\tau>0$ and for all $x$ in $X$ such that $d\left(f x, f^{2} x\right)>0$. Then $f$ has property $P$ provided that $f$ is orbitally continuous on $X$.

Proof. First we show that $F(f) \neq \emptyset$. Let $x_{0} \in X$. Define a sequence $\left\{x_{n}\right\}_{n \in \mathbb{N}}$ in $X$, such that $x_{n+1}=f x_{n}$, for all $n \in \mathbb{N} \cup\{0\}$. Denote $\gamma_{n}=$ $d\left(x_{n}, x_{n+1}\right)$ for all $n \in \mathbb{N} \cup\{0\}$. If there exists $n_{0} \in \mathbb{N} \cup\{0\}$ for which $x_{n_{0}+1}=$ $x_{n_{0}}$, then $f x_{n_{0}}=x_{n_{0}}$ and the proof is finished. Suppose that $x_{n+1} \neq x_{n}$, for all $n \in \mathbb{N} \cup\{0\}$. Using (6.3.1), we obtain

$$
\begin{aligned}
F\left(\gamma_{n}\right) & =F\left(d\left(x_{n}, x_{n+1}\right)\right)=F\left(d\left(f x_{n-1}, f^{2} x_{n-1}\right)\right) \\
& \leq F\left(d\left(x_{n-1}, f x_{n-1}\right)\right)-\tau=F\left(d\left(f x_{n-2}, f^{2} x_{n-2}\right)\right)-\tau \\
& \leq F\left(d\left(x_{n-2}, f x_{n-2}\right)\right)-2 \tau \leq \ldots \\
& \leq F\left(d\left(x_{1}, x_{2}\right)\right)-(n-1) \tau \\
& =F\left(d\left(f x_{0}, f^{2} x_{1}\right)\right)-(n-1) \tau \leq F\left(d\left(x_{0}, x_{1}\right)\right)-n \tau \\
& =F\left(\gamma_{0}\right)-n \tau .
\end{aligned}
$$

for every $n \in \mathbb{N} \cup\{0\}$. By taking limit as $n \rightarrow \infty$ in above inequality, we obtain that $\lim _{n \rightarrow \infty} F\left(\gamma_{n}\right)=-\infty$, that together with (C2) gives $\lim _{n \rightarrow \infty} \gamma_{n}=0$. 
From $(\mathrm{C} 3)$, there exist $k \in(0,1)$ such that $\lim _{n \rightarrow \infty} \gamma_{n}^{k} F\left(\gamma_{n}\right)=0$. Note that

$$
\begin{aligned}
\gamma_{n}^{k} F\left(\gamma_{n}\right)-\gamma_{n}^{k} F\left(\gamma_{0}\right) & \leq \gamma_{n}^{k}\left(F\left(\gamma_{0}\right)-n \tau\right)-\gamma_{n}^{k} F\left(\gamma_{0}\right) \\
& =-\gamma_{n}^{k} n \tau \leq 0
\end{aligned}
$$

On taking limit as $n \rightarrow \infty$, we get $\lim _{n \rightarrow \infty} n \gamma_{n}^{k}=0$. Hence there exists $n_{1}$ such that $n \gamma_{n}^{k} \leq 1$ for all $n \geq n_{1}$. Consequently $\gamma_{n} \leq 1 / n^{1 / k}$ for all $n \geq n_{1}$. Now for integers $m>n \geq 1$ such that

$$
\begin{aligned}
d\left(f^{n} x_{0}, f^{m} x_{0}\right)= & d\left(x_{n}, x_{m}\right) \leq d\left(x_{n}, x_{n+1}\right)+d\left(x_{n+1}, x_{n+2}\right)+\ldots \\
& +d\left(x_{m-1}, x_{m}\right) \\
< & \sum_{i=n}^{\infty} \gamma_{i} \leq \sum_{i=n}^{\infty} \frac{1}{i^{\frac{1}{k}}}<\infty
\end{aligned}
$$

This shows that $\left\{f^{n} x_{0}\right\}_{n \in \mathbb{N}}$ is a Cauchy sequence. Since $\left\{f^{n} x_{0}: n \in \mathbb{N}\right\} \subseteq$ $O\left(x_{0}\right) \subseteq X$ and $X$ is complete which implies that there exists an $x$ in $X$ such that $\lim _{n \rightarrow \infty} f^{n} x_{0}=x$. Since $f$ is orbitally continuous at $x$, so $x=\lim _{n \rightarrow \infty} f^{n} x_{0}=$ $f\left(\lim _{n \rightarrow \infty} f^{n-1} x_{0}\right)=f x$. Hence $f$ has fixed point and $F\left(f^{n}\right)=F(f)$ is true for $n=1$. Now assume $n>1$. Suppose on contrary that $u \in F\left(f^{n}\right)$ but $u \notin F(f)$, then $d(u, f u)=\alpha>0$. Now consider

$$
\begin{aligned}
F(\alpha) & =F\left(d(u, f u)=F\left(d\left(f\left(f^{n-1} u\right), f^{2}\left(f^{n-1} u\right)\right)\right)\right. \\
& \leq F\left(d\left(f^{n-1} u, f^{n} u\right)\right)-\tau \\
& \leq F\left(d\left(f^{n-2} u, f^{n-1} u\right)\right)-2 \tau \\
& \leq \cdots \leq F(d(u, f u))-n \tau .
\end{aligned}
$$

Thus $F(\alpha) \leq \lim _{n \rightarrow \infty} F(d(u, f u))-n \tau=-\infty$. Hence $F(\alpha)=-\infty$. By $(\mathrm{C} 2)$ $\alpha=0$, a contradiction. So $u \in F(f)$.

Theorem 6.3.4 Let $(X, \preceq)$ be a partially ordered set such that there exists a complete metric $d$ on $X$ and $f, g$ self mappings on $X$. Further assume that $f, g$ are weakly increasing and satisfy

$$
\tau+F(d(f x, g y)) \leq F(d(x, y))
$$


for some $\tau>0$, for all $x, y$ in $X$ such that $\min \{d(f x, g y), d(x, y)\}>0$. Then $f$ and $g$ have property $Q$ provided that $X$ has sequential limit comparison property.

Proof. By Theorem 6.2.3, $f$ and $g$ have common fixed point. Suppose on contrary that

$$
u \in F\left(f^{n}\right) \cap F\left(g^{n}\right)
$$

but $u \notin F(f) \cap F(g)$, then there are three possibilities (a) $u \in F(f) \backslash F(g)$, (b) $u \in F(g) \backslash F(f)($ c) $u \notin F(f)$ and $u \notin F(g)$. Without loss of generality let $u \notin F(g)$, that is $d(u, g u)=\alpha>0$, so we get

$$
\begin{aligned}
F(\alpha) & =F\left(d(u, g u)=F\left(d\left(f\left(f^{n-1} u\right), g\left(g^{n} u\right)\right)\right)\right. \\
& \leq F\left(d\left(f^{n-1} u, g^{n} u\right)\right)-\tau \\
& \leq F\left(d\left(f^{n-2} u, g^{n-1} u\right)\right)-2 \tau \\
& \leq \cdots \leq F(d(u, g u))-n \tau .
\end{aligned}
$$

As $\lim _{n \rightarrow \infty} F(d(u, g u)-n \tau=-\infty$, so we have $F(\alpha)=-\infty$. By $(\mathrm{C} 2) \alpha=0$, a contradiction. Hence $u \in F(g) \cap F(f)$.

\subsection{Fixed points of generalized contractions}

In this section, we introduce a notion of generalized $F$-contraction mappings which is used to prove a fixed point result for generalized nonexpansive mappings on star shaped subsets of normed linear spaces. Some theorems on invariant approximations in normed linear spaces are deduced. Our results extend, unify and generalize comparable results in [22, 34, 77, 92]. Some illustrative examples are also presented. 
Motivated by the work of Wardowski [129] and by Theorem 4 of [1], we give the following definition.

Definition 6.4.1 Let $(X, d)$ be a metric space and $F \in \digamma$. A mapping $f: X \rightarrow X$ is said to be a generalized $F$-contraction if there exists a $\tau>0$ such that

$$
d\left(f x, f^{2} x\right)>0 \Rightarrow \tau+F\left(d\left(f x, f^{2} x\right)\right) \leq F(d(x, f x))
$$

for all $x \in X$.

Definition 6.4.2 Let $(X, d)$ be a metric space and $F \in \digamma$. A mapping $f: X \rightarrow X$ is said to be $F$-nonexpansive if

$$
d(f x, f y)>0 \Rightarrow F(d(f x, f y)) \leq F(d(x, y))
$$

for all $x, y \in X$.

Remark 6.4.3 It follows from condition $\mathrm{C} 1$ that if $F \in \digamma$ and $f$ is an $F$-nonexpansive self-mapping of a metric space $(X, d)$, then $f$ is nonexpansive (recall that $f$ is nonexpansive provided that $d(f x, f y) \leq d(x, y)$ for all $x, y \in X)$. Conversely, it is clear, by $\mathrm{C} 1$, that if $f$ is a nonexpansive selfmapping of a metric space $(X, d)$, then $f$ is $F$-nonexpansive for all $F \in \digamma$.

By considering different choices of mappings $F$ in (6.4.1) and (6.4.2), we obtain a variety of contractions. For details we refer to [129] and the following examples.

Example 6.4.4 Let $(X, d)$ be a metric space, $F \in \digamma$ and $G: \mathbb{R}_{+} \rightarrow$ $\mathbb{R}$ be given by $G(\alpha)=F(\alpha)-\tau$, where $\tau>0$. It is clear that $G \in \digamma$. Now, if $f: X \rightarrow X$ is a generalized $F$-contraction, then it is a generalized $G$-contraction because for any $x, y \in X$ with $d\left(f x, f^{2} x\right)>0$, we have

$$
\begin{aligned}
\tau+G\left(d\left(f x, f^{2} x\right)\right) & =F\left(d\left(f x, f^{2} x\right)\right) \\
& \leq F(d(x, f x))-\tau=G(d(x, f x))
\end{aligned}
$$

Similarly, if $f$ is an $F$-contraction, then it is a $G$-contraction. Furthermore, 
if $f$ is $F$-nonexpansive then

$G(d(f x, f y))=F(d(f x, f y))-\tau \leq F(d(x, y))-2 \tau \leq G(d(x, y))-\tau \leq G(d(x, y))$,

whenever $d(f x, f y)>0$, which shows that $f$ is $G$-nonexpansive. Finally, note that taking $G(\alpha)=\ln (\alpha)$ in (6.4.3), we deduce that $f$ is nonexpansive. Example 6.4.5 Let $(X, d)$ be a metric space, let $F_{1}: \mathbb{R}_{+} \rightarrow \mathbb{R}$ be given by $F_{1}(\alpha)=\ln (\alpha)$ and let $f: X \rightarrow X$ a generalized $F$-contraction. Since $F_{1} \in \digamma$, then (6.4.1) becomes

$$
\tau+\ln \left(d\left(f x, f^{2} x\right)\right) \leq \ln (d(x, f x))
$$

whenever $d\left(f x, f^{2} x\right)>0$, which implies

$$
\ln \frac{d\left(f x, f^{2} x\right)}{d(x, f x)} \leq-\tau, \text { that is, } \frac{d\left(f x, f^{2} x\right)}{d(x, f x)} \leq e^{-\tau},
$$

and thus $d\left(f x, f^{2} x\right) \leq e^{-\tau} d(x, f x)$. Hence our definition is more general than those given in [77] and [124].

If we take $F_{2}(\alpha)=\ln (\alpha)+\alpha$, it is clear that $F_{2} \in \boldsymbol{\digamma}$, and then (6.4.1) becomes

$$
\tau+\ln \left(d\left(f x, f^{2} x\right)\right)+d\left(f x, f^{2} x\right) \leq \ln (d(x, f x))+d(x, f x)
$$

whenever $d\left(f x, f^{2} x\right)>0$, which implies that

$$
\frac{d\left(f x, f^{2} x\right)}{d(x, f x)} e^{d\left(f x, f^{2} x\right)-d(x, f x)} \leq e^{-\tau},
$$

i.e.,

$$
d\left(f x, f^{2} x\right) \leq \frac{e^{-\tau}}{e^{d\left(f x, f^{2} x\right)-d(x, f x)}} d(x, f x) .
$$

Definition 6.4.6 Let $C$ be a closed subset of metric space $(X, d)$. Then $f: C \rightarrow C$ is called compact if for every bounded subset $A$ of $C, \overline{f(A)}$ is compact in $C$. 
Definition 6.4.7 If $f: X \rightarrow X$ is a mapping with $f(C) \subseteq C$, then $C$ is called an $f$-invariant subset of $X$.

Definition 6.4.8 Let $C$ be a subset of metric space $(X, d)$. As usual, for any $x \in X$, we define

$$
d(x, C)=\inf \{d(x, y): y \in C\}
$$

and

$$
P_{C}(x)=\{y \in C: d(x, y)=d(x, C)\} .
$$

$P_{C}(x)$ is called the set of best approximations of $x$ from $C$. If for each $x \in X$, $P_{C}(x)$ is nonempty then $C$ is called proximinal. Observe that if $C$ is closed, then $P_{C}(x)$ is also closed.

Definition 6.4.9 Let $E$ be a linear space over $\mathbb{R}$. A subset $C$ of $E$ is called star-shaped if there exists at least one point $z \in C$ such that $t z+(1-t) x \in C$ for all $x \in C$ and $0<t<1$. In this case $z$ is called a star centre of $C$.

Let $(X, d)$ be a metric space, $C$ be a closed subset of $X$ and $f: C \rightarrow C$ be a self-mapping. For each $x \in C$, the set $O(x)=\left\{x, f x, \ldots, f^{n} x, \ldots\right\}$ is called the orbit of $x$ (compare [58]). The mapping $f$ is called orbitally continuous at $p$ if $\lim _{n \rightarrow \infty} f^{n} x=p$ implies $\lim _{n \rightarrow \infty} f^{n+1} x=f p$, and $f$ is orbitally continuous on a set $C$ if $f$ is orbitally continuous for all $p \in C$.

In the following a normed linear space $(E,\|\|$.$) will be simply denoted$ by $E$ if no confusion arises. Furthermore, by a complete subset of a normed linear space $E$ we will mean a subset $A$ of $E$ such that the restriction to $A$ of the metric induced on $E$ by its norm is a complete. Of course, every complete subset of a normed linear space is closed, and every closed subset of a Banach space is complete.

Our main result will be proved with the help of the following re-formulation of Theorem 4 of [1].

Theorem 6.4.10 [1] Let $(X, d)$ be a complete metric space, $F \in \digamma$ and $f: X \rightarrow X$ an orbitally continuous generalized $F$-contraction. Then $f$ has a fixed point. 
Theorem 6.4.11 Let $E$ be a normed linear space, $C$ a complete and star-shaped subset of $E$ and $F \in \digamma$. If $f: C \rightarrow C$ is an $F$-nonexpansive mapping and $\overline{f(C)}$ is compact, then $f$ has a fixed point.

Proof. We first note that, by Remark 6.4.3, $f$ is nonexpansive on $C$, so it is continuous on $C$.

Now let $z$ be a star centre of $C$. For each $n \geq 1$, define $f_{n}: C \rightarrow C$ by

$$
f_{n} x=\left(1-k_{n}\right) z+k_{n} f x
$$

for all $x \in C$, where $0<k_{n}<1$ and $\lim _{n \rightarrow \infty} k_{n}=1$. From the fact that $f$ is continuous on $C$ it immediately follows that each $f_{n}$ is continuous on $C$.

For any fixed $n \geq 1$ and any $x \in C$, we have

$$
\begin{aligned}
F\left(\left\|f_{n} x-f_{n}^{2} x\right\|\right) & =F\left(\left\|\left(1-k_{n}\right) z+k_{n} f x-f_{n}\left(\left(1-k_{n}\right) z+k_{n} f x\right)\right\|\right) \\
& =F\left(\left\|\left(1-k_{n}\right) z+k_{n} f x-\left(1-k_{n}\right) z-k_{n} f\left(\left(1-k_{n}\right) z+k_{n} f x\right)\right\|\right) \\
& =F\left(\left\|k_{n}\left(f x-f\left(\left(1-k_{n}\right) z+k_{n} f x\right)\right)\right\|\right) .
\end{aligned}
$$

Since $F$ is strictly increasing, with $k_{n}<1$ for each $n \geq 1$, and $f$ is $F$-nonexpansive, we have

$$
\begin{aligned}
F\left(\left\|f_{n} x-f_{n}^{2} x\right\|\right) & <F\left(\left\|f x-f\left(\left(1-k_{n}\right) z+k_{n} f x\right)\right\|\right) \\
& <F\left(\left\|x-\left(\left(1-k_{n}\right) z+k_{n} f x\right)\right\|\right)=F\left(\left\|x-f_{n} x\right\|\right) .
\end{aligned}
$$

Hence

$$
F\left(\left\|x-f_{n} x\right\|\right)-F\left(\left\|f_{n} x-f_{n}^{2} x\right\|\right)>0 .
$$

This implies that there exists $\tau_{n}>0$, such that

$$
0<\tau_{n} \leq F\left(\left\|x-f_{n} x\right\|\right)-F\left(\left\|f_{n} x-f_{n}^{2} x\right\|\right)
$$

Therefore

$$
\tau_{n}+F\left(\left\|f_{n} x-f_{n}^{2} x\right\|\right) \leq F\left(\left\|x-f_{n} x\right\|\right) .
$$

Hence $f_{n}$ is a generalized $F$-contraction for each $n \geq 1$. By Theorem 6.4.10, for each $n \geq 1$ there exists $x_{n} \in C$ such that $f_{n} x_{n}=x_{n}$. Since $\overline{f(C)}$ is 
compact there exist a subsequence $\left\{f x_{n_{i}}\right\}_{i \geq 1}$ of the sequence $\left\{f x_{n}\right\}_{n \geq 1}$, and an $x \in \overline{f(C)}$ such that

$$
x=\lim _{i \rightarrow \infty} f x_{n_{i}} .
$$

(In fact $x \in C$ because $f(C) \subseteq C$ and $C$ is closed).

Since $\lim _{i \rightarrow \infty} k_{n_{i}}=1$ and $x_{n_{i}}=f_{n_{i}} x_{n_{i}}$ for all $i \geq 1$, we deduce that

$$
x=\lim _{i \rightarrow \infty} f x_{n_{i}}=\lim _{i \rightarrow \infty}\left(\left(1-k_{n_{i}}\right) z+k_{n_{i}} f x_{n_{i}}\right)=\lim _{i \rightarrow \infty} f_{n_{i}} x_{n_{i}}=\lim _{i \rightarrow \infty} x_{n_{i}} .
$$

Therefore

$$
f x=\lim _{i \rightarrow \infty} f x_{n_{i}}
$$

We conclude that $x=f x$.

Remark 6.4.12 Note that, by Remark 6.4.3, we can restate the preceding theorem as follows: Let $E$ be a normed linear space (resp. Banach space) and $C$ a complete (resp. closed) and star-shaped subset of $E$. If $f: C \rightarrow C$ is a nonexpansive mapping and $\overline{f(C)}$ is compact, then $f$ has a fixed point.

The following is an example where we can apply Theorem 6.4.11 for every $F \in \digamma$.

Example 6.4.13 Let $\ell_{1}$ be the linear space of all summable sequences of real numbers. Then, the pair $\left(\ell_{1},\|\cdot\|_{1}\right)$ is a (classical) Banach space, where $\|\cdot\|_{1}$ is the norm on $\ell_{1}$ such that for each $\mathbf{x}:=\left\{x_{n}\right\}_{n \geq 1} \in \ell_{1}$,

$$
\|\mathbf{x}\|_{1}=\sum_{n=1}^{\infty}\left|x_{n}\right| .
$$

In the following any element $\mathbf{x}:=\left\{x_{n}\right\}_{n \geq 1}$ of $\ell_{1}$ will be also denoted as

$$
\left(x_{1}, x_{2}, x_{3}, \ldots, x_{n}, \ldots\right)
$$

Let $C$ be the closed unit ball of $\left(\ell_{1},\|\cdot\|_{1}\right)$, i.e.,

$$
C=\left\{\mathbf{x} \in \ell_{1}:\|\mathbf{x}\|_{1} \leq 1\right\}
$$


It is well known that $C$ is a (noncompact) closed subset of $\left(\ell_{1},\|\cdot\|_{1}\right)$. Moreover $C$ is star-shaped with $\mathbf{z}=\mathbf{0}$ a star center of $C$.

Now let $k \in(0,1]$ constant, and define $f: C \rightarrow C$ as

$$
f \mathbf{x}=\left(x_{1}, k x_{2}, 0, \ldots, 0, \ldots\right)
$$

for all $\mathbf{x}:=\left(x_{n}\right)_{n \geq 1} \in \ell_{1}$. Clearly $f$ is nonexpansive on $C$, and hence it is $F$-nonexpansive for any $F \in \digamma$, by Remark 6.4.3.

Furthermore $f(C)$ is homeomorphic to the compact subset of $\mathbb{R}^{2}$,

$$
\left\{(u, v) \in \mathbb{R}^{2}:|u|+\left|\frac{v}{k}\right| \leq 1\right\}
$$

so that $f(C)$ is compact. Hence $f(C)=\overline{f(C)}$ and thus $\overline{f(C)}$ is compact. We have shown that all conditions of Theorem 6.4.11 (compare Remark 6.4.3) are satisfied. Thus $f$ has a fixed point. In fact, the fixed points of $f$ are the elements $\mathbf{x}=\left\{x_{n}\right\}_{n \geq 1}$ of $C$ such that $x_{n}=0$ whenever $n \geq 2$.

Now we give an example of a discontinuous self-mapping $f$ on a compact metric space which is a generalized $F$-contraction but not an $F$-contraction. So the class of generalized $F$-contraction mappings is a bigger than the class of $F$-contraction.

Example 6.4.14 Let $X=\mathbb{R}$, and $C=[0,1]$ be endowed with usual metric. Define a mapping $f: C \rightarrow C$ as $f(x)=1$ if $x \in\{0,1\}$, and $f(x)=x / 2$ if $x \in(0,1)$.

Let $F: \mathbb{R}_{+} \rightarrow \mathbb{R}$ be defined by $F x=\ln x$ for $x \in(0,1)$. Note that

$$
\ln 2+F\left(d\left(f x, f^{2} x\right)\right) \leq F(d(x, f x))
$$

is satisfied for all $x \in C$ whenever $d\left(f x, f^{2} x\right)>0$. Hence $f$ is a generalized $F$-contraction. Clearly $f$ is not continuous at $x=1$ and at $x=0$. Hence $f$ is not an $F$-contraction ( see Remark 2.1 in [129] ) or let $x=0$ and $y=\frac{1}{2}$, then

$$
F\left(d\left(f 0, f \frac{1}{2}\right)\right)=F\left(d\left(1, \frac{1}{4}\right)\right)=F\left(\frac{3}{4}\right)=\ln \left(\frac{3}{4}\right)=\ln 3-2 \ln 2,
$$


while

$$
F\left(d\left(0, \frac{1}{2}\right)\right)=F\left(\frac{1}{2}\right)=\ln \left(\frac{1}{2}\right)=-\ln 2,
$$

and thus

$$
F\left(d\left(0, \frac{1}{2}\right)\right)-F\left(d\left(f 0, f \frac{1}{2}\right)\right)=\ln 2-\ln 3<0 .
$$

Hence there does not exist any $\tau>0$, such that for $x=0$ and $y=\frac{1}{2}$,

$$
\tau+F(d(f x, f y)) \leq F(d(x, y))
$$

is satisfied. Now let $x_{0}=0$, then $f(0)=1$, and $f^{2}(0)=1$. Hence the orbit of $x_{0}$ is the set $O(0)=\{0,1,1, \ldots\}=\overline{O(0)}$ which is compact, and

$$
\lim _{n \rightarrow \infty} f^{n}(0)=1, \lim _{n \rightarrow \infty} f^{n+1}(0)=f 1=1
$$

that is $f$ is orbitally continuous at 1 . Hence, by Theorem $6.4 .11, f$ has a fixed point (in fact $x=1$ is the only fixed point of $f$ ).

\subsection{Invariant approximation results in non- convex subsets of normed spaces}

In the last part, we discuss nonemptiness and existence of fixed points for the set of best approximations of closed subsets of metric spaces and of normed spaces, respectively.

Theorem 6.5.1 Let $(X, d)$ be a metric space. Let $F \in \digamma$ be a continuous mapping and $f: X \rightarrow X$ be $F$-nonexpansive with a fixed point $u \in X$. If $C$ is a closed $f$-invariant subset of $X$ such that $f$ is compact on $C$, then the set $P_{C}(u)$ of best approximations is nonempty.

Proof. Let $r=d(u, C)$. Then, there is a sequence $\left\{y_{n}\right\}_{n \geq 1}$ in $C$ such that $\lim _{n \rightarrow \infty} d\left(u, y_{n}\right)=r$. Since $\left\{y_{n}: n \geq 1\right\}$ is a bounded subset of $C$ and $f$ is compact on $C$, the set $\overline{\left\{f y_{n}: n \geq 1\right\}}$ is a compact subset of $C$, and so 
there exist a subsequence $\left\{f y_{n_{i}}\right\}_{i \geq 1}$ of $\left\{f y_{n}\right\}_{n \geq 1}$ and an $x \in C$ such that $\lim _{i \rightarrow \infty} f y_{n_{i}}=x$. Now,

$$
\begin{aligned}
F(r) & \leq F(d(u, x))=F\left(\lim _{i \rightarrow \infty} d\left(f u, f y_{n_{i}}\right)\right) \\
& =\lim _{i \rightarrow \infty} F\left(d\left(f u, f y_{n_{i}}\right)\right) \leq \lim _{i \rightarrow \infty} F\left(d\left(u, y_{n_{i}}\right)\right) \\
& =F\left(\lim _{i \rightarrow \infty} d\left(u, y_{n_{i}}\right)\right)=F(r) .
\end{aligned}
$$

This implies

$$
F(r)=F(d(u, x)) .
$$

Since $F$ is strictly increasing, we get $r=d(u, x)$. Hence $P_{C}(u)$ is nonempty.

As an application of Theorems 6.4.11 and 6.5.1 we deduce the following. Theorem 6.5.2 Let $E$ be a normed linear space. Let $F \in \digamma$ be a continuous mapping and $f: E \rightarrow E$ be $F$-nonexpansive with a fixed point $u \in E$. If $C$ is a complete $f$-invariant subset of $E$ such that $f$ is compact on $C$, and $P_{C}(u)$ is a star-shaped set, then $f$ has a fixed point in $P_{C}(u)$.

Proof. By Theorem 6.5.1, $P_{C}(u)$ is nonempty. We show that $P_{C}(u)$ is $f$-invariant. To this end, let $y \in P_{C}(u)$ and set $r=d(u, C)$, then

$$
\begin{aligned}
F(r) & \leq F(d(u, f y))=F(d(f u, f y)) \\
& \leq F(d(u, y))=F(r) .
\end{aligned}
$$

This implies

$$
F(r)=F(d(u, f y)) .
$$

Since $F$ is strictly increasing, we get $r=d(u, f y)$. So $f y \in P_{C}(u)$. This proves that $P_{C}(u)$ is $f$-invariant, so $f: P_{C}(u) \rightarrow P_{C}(u)$ is $F$-nonexpansive. Now observe that if $C$ is complete then $P_{C}(u)$ is also complete. Hence $P_{C}(u)$ is star-shaped and complete, and $\overline{f\left(P_{C}(u)\right)}$ is compact, so, by Theorem 6.4.11, there exists $x \in P_{C}(u)$ such that $f x=x$. 
Remark 6.5.3 As in the case of Theorem 6.4.11 (see Remark 6.4.3), the preceding theorem can be restated as follows: Let $E$ be a normed linear space (resp. Banach space) and let $f: E \rightarrow E$ be nonexpansive with a fixed point $u \in E$. If $C$ is a complete (resp. closed) $f$-invariant subset of $E$ such that $f$ is compact on $C$, and $P_{C}(u)$ is a star-shaped set, then $f$ has a fixed point in $P_{C}(u)$.

We conclude the chapter illustrating Theorem 6.5.2 with the following example.

Example 6.5.4 Let $\left(\ell_{1},\|\cdot\|_{1}\right)$ be the Banach space of Example 6.4.13. Define $f: \ell_{1} \rightarrow \ell_{1}$ as

$$
f \mathbf{x}=\left(x_{1}, k x_{2}, 0, \ldots, 0, \ldots\right),
$$

for all $\mathbf{x}:=\left\{x_{n}\right\}_{n \geq 1} \in \ell_{1}$, with $k \in(0,1]$. Let $F \in \digamma$ be continuous. Since $f$ is nonexpansive, it follows from Remark 5 that it is $F$-nonexpansive. Of course, $f$ has fixed points. In fact

$$
F i x(f)=\left\{\mathbf{x}:=\left\{x_{n}\right\}_{n \geq 1} \in \ell_{1}: x_{n}=0 \text { for all } n \geq 2\right\}
$$

As in Example 7.2 .9 , let $C$ be the closed unit ball of $\left(\ell_{1},\|\cdot\|_{1}\right)$. We know that $C$ is a closed, and thus complete, $f$-invariant subset of $E$ such that $f$ is compact on $C$.

Then, if we choose $\mathbf{x}:=\left\{x_{n}\right\}_{n \geq 1} \in F i x(f)$ such that $\left|x_{1}\right|>1$, we deduce that $P_{C}(\mathbf{x})=(1,0,0, \ldots, 0, \ldots)$ if $x_{1}>1$, and $P_{C}(\mathbf{x})=(-1,0,0, \ldots, 0, \ldots)$ if $x_{1}<-1$. Therefore, $P_{C}(u)$ is trivially star-shaped. Thus, all conditions of Theorem 6.5.2 (compare Remark 6.5.3) have been verified. 


\section{Chapter 7}

\section{Discussion of the Obtained Results and Conclusions}

The development of soft topology is based on basic soft set operations. This is the reason that a lot of research activity around soft set operations has been seen in recent years. The concepts of soft equality, soft union and soft intersection are the basic ingredients to prove Demorgan's laws in the setup of soft set theory. The soft null set and soft universal sets are also important to study soft topology. It has been observed that several basic properties in [87] do not hold true in general. This was the basic motivation for Chapter 2 of this thesis.

We introduced the concepts of $g$-null soft set and $g$-soft subset of a soft set along with the notion of $g$-soft equality relation $\approx_{g}$ between the soft sets. It is shown that $g$-soft equality relation $\approx_{g}$ generalizes existing comparable concepts about equality of soft sets. Moreover we gave example to show that $\approx_{g}$ gives rise to the bigger class of soft subsets which ultimately will refine the bases for soft topological spaces. Furthermore, we give algebraic structure (lattice structure with order $\preceq_{s}$ on the class of soft sets) with $g$-soft equality relation $\approx_{g}$ and already existing operations on union and intersection of soft sets. It is proved that order relation $\preceq_{s}$ is dependence relation and $g$-soft 
equality relation is an equivalence relation on the collection of soft sets. Some examples have been provided as illustrations and for comparisons. The new operations on soft sets will be important basis for the further developments on soft set theory.

Recently, Tridiv [100] and Mahanta [85] studied the concept of fuzzy soft topological spaces. Chapter 3 deals with our initiative of the study of fixed points in the framework of fuzzy soft set theory. In order to develop this theory, it was natural to develop some basic machinery to deal with this new subject. The concept of fuzzy soft mapping was central to this chapter. We also studied some basic properties of fuzzy soft elements. We also proved a fuzzy soft Cantor intersection theorem for the class of fuzzy soft closed subsets of fuzzy soft compact topological space. Based on these concepts, we were able to obtain fixed points of fuzzy soft mappings.

Das and Samanta [29, 28] coined the notion of soft real set, soft real numbers and studied their properties. Along these directions, they introduced the concept of a soft metric. In Chapter 4 we introduced the concept of a soft contraction mappings and then proved soft contraction theorem. We also obtained a fixed point result for a soft contraction mapping on soft closed subsets of complete soft metric space. These results can be viewed as a foundation of soft metric fixed point theory. Considering these results as a starting point, soft metric fixed point theory can be developed further and we believe that this will attract mathematicians working in metric fixed point theory to explore this new dimension of research to expand the boundaries of metric fixed point theory.

The simplicity and usefulness of Banach contraction principle has inspired many researchers to analyze it further. One of the deepest generalization and modification of this principle is well known Caristi fixed point theorem. Strength of this theorem lies in the fact that it characterizes the completeness of a metric space. The fixed point theory of multivalued mappings is a powerful tool for the study of those problems of computational mathematics 
which can be formulated as fixed point inclusion for an appropriate multivalued mapping. In Chapter 5, employing concept of Caristi type multivlaued mappings, an existence of fixed point of such mapping is obtained in the setup of complete fuzzy metric spaces. This fixed point theorem provides a characterization of fuzzy metric completeness in the case of continuous t-norms greater than or equal to the Lukasiewicz t-norm.

The results presented in Chapter 6 can be viewed as unification, extension and generalizations of several comparable results in existing literature with supporting examples. We introduced a notion of a generalized $F-$ contraction and generalized $F$ - nonexpansive mappings. We also presented an example of a discontinuous mapping defined on an infinite dimensional Banach space which showed that our concept is a substantial generalization of an $F$-contraction mapping. Some best approximation results as an application of our fixed point results are also obtained. We extended the concept of $F$-contraction mapping given in [129] to two mappings and then obtained common fixed point results in the setup of partially ordered sets equipped with complete metric. As an application of the results presented therein, periodic point property of two mappings was also investigated.

Following is a brief summary of findings of our study:

i) We defined some new operations on soft sets that will be important basis for the further developments on soft set theory. Based on the definitions and results given in Chapter 2, some more properties on soft sets can be established and existing soft topological concepts can be modified in the light of these results. Consequently, several existing results in soft topological spaces can be studied employing our definitions in this chapter. The scope of these results is not limited to the study of soft topological spaces. The similar observation can also provide a basis to revisit the existing concepts of basic operations in fuzzy soft set and intuitionistic fuzzy soft set theory. These findings could add a new dimension to the present study of soft and fuzzy soft topological 
spaces.

ii) We put forward the notion of fuzzy soft mappings based on the theory of fuzzy soft element of fuzzy soft set and fuzzy soft topological space. In Chapter 3 we study fixed points of fuzzy soft mappings. Employing these results, one can further study fixed point theory in the framework of fuzzy soft set theory. These results can be extended further in different directions. For instance, it will be a matter of great interest to study the same problem in intuitionistic fuzzy soft set theory. The results proved in this chapter are of basic nature so they can be considered as a foundation results to develop fixed point theory for fuzzy soft mappings.

iii) In Chapter 4 we introduce the notion of soft contraction mapping based on the theory of soft element of soft metric space. We study fixed points of soft contraction mappings and obtain, among others results, a theorem of Banach contraction principle type. Employing these results, we can further study fixed point theory in the framework of soft metric spaces. Some constructive examples are provided to support our findings in this direction. We hope that our results will open some new avenues of research. For instance, different contractive conditions can be considered to extend the results presented herein. A study of common fixed point theory based on our recent investigation could lead to a new area of research. A soft fixed points of soft multivalued mappings in the framework of soft metric spaces can also be a fascinating field of research.

iv) Based on the concept of Caristi's multivalued mappings in complete fuzzy metric spaces, an issue of characterization of completeness of domain of the mapping is addressed in Chapter 5. Example is provided to support the results presented herein. These results provide a good 
platform to intersect fixed point theorems of multivalued mappings with properties of fuzzy metric spaces.

v) The results proved in Chapter 6 lead to different directions and aspect of metric fixed point theory. This work can further be extended to fixed point theory of nonexpansive multivalued mappings. Reformulation of our results replacing a metric space by some generalized metric structure could be considered as a valuable addition to present fixed point results. In short, these results can be extended to different directions. 


\section{Bibliography}

[1] M. Abbas, B. Ali and S. Romaguera, Fixed and periodic points of generalized contractions in metric spaces, Fixed Point Theory Appl., 2013, 2013:243.

[2] M. Abbas, M.A. Khamsi, and A.R. Khan, Common fixed point and invariant approximation in hyperbolic ordered metric spaces, Fixed Point Theory Appl., 2011, 2011:25 doi: 10. 1186/1687-1812-2011-25.

[3] M. Abbas, T. Nazir and S. Radenović, Common fixed points of four maps in partially ordered metric spaces, Applied Math. Lett., 24 (2011), 1520-1526.

[4] Ö. Acar and I. Altun, Some generalizations of Caristi type fixed point theorem on partial metric spaces, Filomat, $26: 4$ (2012), 833-837.

[5] R.P. Agarwal, D.O. O'Regan and N. Shahzad, Fixed point theorem for generalized contractive maps of Meir-Keeler type, Math. Nach., 276 (2004), 3-22.

[6] B. Ahmad and A. Kharal, On fuzzy soft sets, Adv. Fuzzy Syst., 2 (2009), $1-6$.

[7] H. Aktaş and N. Çağman, Soft sets and soft groups, Inform. Sci., 177 (2007), 2726-2735. 
[8] M.A. Al-Thagafi, Common fixed points and best approximation, J. Approx. Theory, 85 (1996), 318-320.

[9] M.I. Ali, F. Feng, X. Liu, W.K. Min and M. Shabir, On some new operations in soft set theory, Comput. Math. Appl., 57 (2009), 15471553.

[10] M.I. Ali and M. Shabir, Comments on De Morgan's law in fuzzy soft sets, Int. J. Fuzzy Math., 18 (2010), 679-686.

[11] I. Altun, B. Damjanović and D. Djorić, Fixed point and common fixed point theorems on ordered cone metric spaces, Appl. Math. Lett., 23 (2010), 310-316.

[12] I. Altun and H. Simsek, Some fixed point theorems on ordered metric spaces and application, Fixed Point Theory Appl., 2010 (2010) Article ID $621492,1-17$.

[13] A. Amini-Harandi and H. Emami, A fixed point theorem for contraction type maps in partially ordered metric spaces and application to ordinary differential equations, Nonlinear Anal., 72 (2010), 2238-2242.

[14] Q.H. Ansari, Metric Spaces: Including Fixed Point Theory and SetValued Maps, Alpha Science, 2010.

[15] I. Arandjelovic, Z. Kadelburg and S. Radenovic, Boyd-Wong-type common fixed point results in cone metric spaces, Appl. Math. Comput., 217 (2011), 7167-7171.

[16] J.P. Aubin and J. Siegel, Fixed point and stationary points of dissipative multi-valued maps, Proc. Amer. Math. Soc., 78 (1980), 391-398.

[17] K.V. Babitha and J.J. Sunil, Soft set relations and functions, Comput. Math. Appl., 60 (2010), 1840-1849. 
[18] S. Banach, Sur les opérations dans les ensembles abstraits et leur applications aux équations intégrales, Fund. Math., 3 (1922), 133-181.

[19] I. Beg and M. Abbas, Coincidence point and invariant approximation for mappings satisfying generalized weak contractive condition, Fixed Point Theory Appl., 2006 (2006), Article ID 74503, pages 1-7.

[20] D.W. Boyd and J.S.W. Wong, On nonlinear contractions, Proc. Amer. Math. Soc., 20 (1969), 458-464.

[21] A. Branciari, A fixed point theorem for mappings satisfying a general contractive condition of integral type, Int. J. Math. Math. Sci., 29 (2002), 531-536.

[22] B. Brosowski, Fixpunktsätze in der Approximationstheorie, Mathematica (Cluj), 11 (34) (1969), 195-220.

[23] N. Cagman, S. Karatas and S. Enginoglu, Soft topology, Comput. Math. Appl., 62 (2003), 351-358.

[24] J. Caristi, Fixed point theorems for mappings satisfying inwardness conditions, Trans. Amer. Math. Soc., 215 (1976), 241-251.

[25] Y.J. Cho, M. Grabiec and V.Radu, On Nonsymmetric Topological and Probabilistic Structures, Nova Science Publisher, New York, 2006.

[26] L. Ciric, M. Abbas, R. Saadati and N. Hussain, Common fixed points of almost generalized contractive mappings in ordered metric spaces, Appl. Math. Comput., 217 (2011), 5784-5789.

[27] H. Covitz and S.B. Nadler Jr., Multi-valued contraction mappings in generalized metric space, Israel J. Math., 8 (1970), 5-11.

[28] S. Das and S.K. Samanta, Soft metric, Ann. Fuzzy Math. Inform. 6 (2013), 77-94. 
[29] S. Das and S.K. Samanta, Soft real sets, soft real numbers and their properties, J. Fuzzy Math., 20 (2012), 551-576.

[30] A. Deb Ray and P.K. Saha, Fixed point theorems on generalized fuzzy metric spaces. Hacettepe J. Math. Stat. 39 (2010), 1-9.

[31] Z.K. Deng, Fuzzy pseudo-metric spaces, J. Math. Anal. Appl., 86 (1982), 74-95.

[32] B. Dinda and T.K. Samanta, Relations on intuitionistic fuzzy soft sets, Gen. Math. Notes, 1 (2010), 74-83.

[33] J.G. Dix and G.L. Karakostas, A fixed point theorem for S-type operators on Banach spaces and its applications to boundary-value problems, Nonlinear Anal., 71 (2009), 3872-3880.

[34] W.G. Dotson, Fixed point theorems for non-expansive mappings on starshaped subsets of Banach spaces, J. London Math. Soc., 4 (1972), 408410.

[35] S.K. Dusmanta, An application of fuzzy soft relation in decision making problems, Int. J. Math. Trends Tech., 3, 2012.

[36] I. Ekeland, Nonconvex minimization problems, Bull. Amer. Math. Soc., 1 (1979), 443-474.

[37] M.S. El Naschie, On a fuzzy Khaler-like manifold which is consistent with two slit experiment, Int. J. Nonlinear Sci. Numer. Simul., 6 (2005), 95-98.

[38] M.A. Erceg, Metric spaces in fuzzy set theory, J. Math. Anal. Appl., 69 (1979), 205-230.

[39] J.X. Fang, On fixed point theorems in fuzzy metric spaces, Fuzzy Sets Syst., 46 (1992), 107-113. 
[40] F. Feng, Y.B. Jun, X.Y. Liu and L.F. Li, An adjustable approach to fuzzy soft set based decision making, J. Comput. Appl. Math., 234 (2009), 10-20.

[41] F. Feng, Y.B. Jun and X. Zhao, Soft semirings, Comput. Math. Appl., 56 (2008), 2621-2628.

[42] F. Feng, C.X. Li, B. Davvaz and M.I. Ali, Soft sets combined with fuzzy sets and rough sets: a tentative approach, Soft Comput., 14 (2010) 8999-9911.

[43] Y. Feng and S. Liu, Fixed point theorems for multi-valued contractive mappings and multi-valued Caristi type mappings, J. Math. Anal. Appl., 317 (2006), 103-112.

[44] F. Feng and X. Liu, Soft rough sets with applications to demand analysis. In: Int. Workshop Intell. Syst. Appl., ISA 2009, 23-24 May 2009, Wuhan, China. IEEE, (2009), 1-4.

[45] F. Feng, X. Liu, V. Leoreanu-Fotea, and Y.B. Jun, Soft sets and soft rough sets, Inf. Sci. 181 (2011), 1125-1137.

[46] A. George and P. Veeramani, On some results in fuzzy metric spaces, Fuzzy Sets Syst., 64 (1994), 395-399.

[47] A. George and P. Veeramani, On some results of analysis for fuzzy metric spaces, Fuzzy Sets Syst., 90 (1997), 365-368.

[48] M. Grabiec, Fixed points in fuzzy metric spaces, Fuzzy Sets Syst., 27 (1983), 385-389.

[49] V. Gregori, S. Morillas and A. Sepena, Examples of fuzzy metrics and applications, Fuzzy Sets Syst., 170 (2011), 95-111. 
[50] V. Gregori and S. Romaguera, Some properties of fuzzy metric spaces, 115 (2000), 485-489.

[51] V. Gregori and A. Sapena, On fixed-point theorems in fuzzy metric spaces, Fuzzy Sets Syst., 125 (2002), 245-252.

[52] L. Habiniak, Fixed point theorems and invariant approximations, J. Approx. Theory, 56 (1989), 241-244.

[53] O. Hadžić and E. Pap, Fixed Point Theory in Probabilistic Metric Spaces, Kluwer Academic Publishers, 2001.

[54] J. Harjani and K. Sadarangani, Fixed point theorems for weakly contractive mappings in partially ordered sets, Nonlinear Anal., 71 (2009), 3403-3410.

[55] T. Herawan and M.M. Deris, On multi-soft sets construction in information systems. In: Emerging Intelligent Computing Technology and Applications with Aspects of Artificial Intelligence: 5th Int. Conf. Intell. Comput., ICIC 2009 Ulsan, South Korea, September 16-19, 2009. Springer, U. T. H. O. Malaysia, (2009), 101-110.

[56] T. Herawan, A.N.M. Rose and M.M. Deris, Soft set theoretic approach for dimensionality reduction. In: Database Theory and Application: International Conference, DTA 2009, Jeju Island, Korea, December 10-12, 2009. Springer, (2009), 171-178.

[57] T.L. Hicks and M.D. Humphries, A note on fixed-point theorems, J. Approx. Theory, 34 (1982), 221-225.

[58] T.L. Hicks and B.E. Rhoades, A Banach type fixed point theorem, Math. Japon., 24 (1979), 327-330.

[59] L.G. Huang and X. Zhang, Cone metric spaces and fixed point theorem of contractive maps, J. Math. Anal. Appl., 332 (2007), 1467-1475. 
[60] L.G. Huang and X. Zhang, Cone metric spaces and fixed point theorems of contractive mappings, J. Math. Anal. Appl., 332 (2007), 1468-1476.

[61] N. Hussain, D. O'Regan and R.P. Agarwal, Common fixed point and invariant approximation results on non-star-shaped domain, Georgian Math. J., 12 (2005), 659-669.

[62] J. Jachymski, Caristi's fixed point theorem and selections of set-valued contractions, J. Math. Anal. Appl., 227 (1998), 55-67.

[63] J. Jachymski, Converses to fixed point theorems of Zeremlo and Caristi, Nonlinear Anal., 52 (2003), 1455-1463.

[64] G.S. Jeong and B.E. Rhoades, Maps for which $F(T)=F\left(T^{n}\right)$, Fixed Point Theory Appl., 6 (2005), 87-131.

[65] Y. Jiang, Y. Tang, Q. Chen, J. Wang and S. Tang, Extending soft sets with description logics, Comput. Math. Appl., 59 (2009), 2087-2096.

[66] Y.B. Jun, Soft BCK/BCI-algebras. Comput. Math. Appl., 56 (2008), 1408-1413.

[67] Y.B. Jun, K.J. Lee and A. Khan, Soft ordered semigroups, Math. Logic Quart. 56 (2010), 42-50.

[68] Y.B. Jun, K.J. Lee and C. H. Park, Soft set theory applied to ideals in d-algebras. Comput. Math. Appl., 57 (2009), 367-378.

[69] Y.B. Jun and C.H. Park, Applications of soft sets in ideal theory of BCK/BCI-algebras, Inform. Sci., 178 (2008), 2466-2475.

[70] G. Jungck, Commuting mappings and fixed points, Amer. Math. Monthly, 83 (1976), 261-263.

[71] G. Jungck, Common fixed point for noncontinuous nonself maps on nonmetric spaces, Far. East J. Math. Sci., 4 (1996), 199-215. 
[72] Z. Kadelburg, M. Pavlović and S. Radenović, Common fixed point theorems for ordered contractions and quasicontractions in ordered cone metric spaces, Comput. Math. Appl., 59 (2010), 3148-3159.

[73] S. Kakutani, A generalization of Brouwer's fixed point theorem, Duke Math. J., 8 (1941), 457-459.

[74] O. Kaleva and S. Seikkala, On Fuzzy metric spaces, Fuzzy Sets Syst., 12 (1984), 215-229.

[75] R. Kannan, Some results on fixed points, Bull. Calcutta Math. Soc., 60 (1968), 71-76.

[76] E. Karapinar, Generalizations of Caristi Kirk's theorem on partial metric spaces, Fixed Point Theory Appl., 2011, 2011:4.

[77] L.A. Khan and A.R. Khan, An extension of Brosowski-Meinardus theorem on invariant approximation, J. Approx. Theory Appl., 11 (1993), Number 4, DOI: 10.1007/BF02836824.

[78] W.A. Kirk, Caristi's fixed-point theorem and metric convexity, Colloq. Math., 36 (1976), 81-86.

[79] Y.K. Kim and W.K. Min, Full soft sets and full soft decision systems, J. Int. Fuzzy Syst., to appear. DOI: 10.3233/IFS-130783.

[80] I. Kramosil and J. Michalek, Fuzzy metric and statistical metric spaces, Kybernetika, 11 (1975), 326-334.

[81] Z. Kong, L. Gao, L. Wang snf S. Li, The normal parameter reduction of soft sets and its algorithm, Comput. Math. Appl., 56 (2008), 3029-3037.

[82] K. Latrach, M.A. Taoudib and A Zeghalc, Some fixed point theorems of the Schauder and the Krasnosel'skii type and application to nonlinear transport equations, J. Differ. Equations, 221 (2006), 256-271. 
[83] F. Li, Notes on the soft operations, ARPN J. Syst. Soft., 1 (6) (2011), 205-208.

[84] Y. Liu and Z. Li, Coincidence point theorems in probabilistic and fuzzy metric spaces, Fuzzy Sets Syst., 158 (2007), 58-70.

[85] J. Mahanta and P.K. Das, Results on fuzzy soft topological spaces, arXiv:1203.0634v1, 2012

[86] P.K. Maji, R. Biswas and A.R. Roy, Fuzzy soft sets, J. Fuzzy Math., 9 (2001), 589-602.

[87] P.K. Maji, R.Biswas and A.R. Roy, Soft set theory, Comput. Math. Appl., 45 (2003), 555-562.

[88] P.K. Maji, R. Biswas and A.R. Roy, Intuitionistic fuzzy soft sets, J. Fuzzy Math., 12 (2004),669-683.

[89] P.K. Maji, A.R. Roy and R. Biswas, An application of soft sets in a decision making problem, Comput. Math. Appl., 44 (2002), 1077-1083.

[90] P. Majumdar and S.K. Samanta, Generalized fuzzy soft sets. Comput. Math. Appl., 59 (2010), 1425-1432.

[91] J.T. Markin, A fixed point theorem for set valued mappings, Bull. Amer. Math. Soc., 74 (1968), 639-640.

[92] G. Meinardus, Invarianz bei linearen Approximationen, Arch. Rational Mech. Anal., 14 (1963), 301-303.

[93] D. Mihet, On the existence and the uniqueness of fixed points of Sehgal contractions, Fuzzy Sets Syst., 156 (2005), 135-141.

[94] D. Mihet, On fuzzy contractive mappings in fuzzy metric spaces, Fuzzy Sets Syst., 158 (2007), 915-921. 
[95] S.N. Mishra, N. Sharma and S.L. Singh, Common fixed points of maps in fuzzy metric spaces, Int. J. Math. Math. Sci., 17 (1994), 253-258.

[96] D.A. Molodtsov, Soft set theory-First results, Comput. Math. Appl., 37 (1999), 19-31.

[97] M.M. Mushrif, S. Sengupta and A.K. Ray, Texture classification using a novel, soft-set theory based classification algorithm, Lecture Notes Comput. Sci., 3851 (2006), 246-254.

[98] S.B. Nadler, Jr., Multivalued contraction mappings, Pacific J. Math., 30 (1969), 475-488.

[99] T.D. Narang, Applications of fixed point theorems to approximation theory, Mat. Vesnik, 36 (1984), 69-75.

[100] T.J. Neog, D.K. Sut and G.C. Hazarika, Fuzzy soft topological spaces, Int. J. Latest Trend Math, 2 (2012), 12 pages.

[101] J.J. Nieto and R.R. López, Contractive mapping theorems in partially ordered sets and applications to ordinary differential equations, Order 22 (2005), 223-239.

[102] X. Pan and Y. Xu, On the algebraic structure of binary lattice-valued fuzzy relations, Soft Comput., 17 (2013), 411-420.

[103] A. Petruşel, and A.Sîntămărian, Single-valued and multi-valued Caristi type operators, Publ. Math. Debrecen, 60 (2002), 167-177.

[104] K. Qin and Z. Hong, On soft equality, J. Comput. Appl. Math., 234 (2010), 1347-1355.

[105] V. Radu, Some fixed point theorems in PM spaces, Lecture Notes Math., vol. 1233, Springer, Berlin, (1985), 125-133. 
[106] E. Rakotch, A note on contractive mappings, Proc. Amer. Math. Soc., 13 (1962), 459-465.

[107] A.C.M. Ran and M.C.B. Reurings, A fixed point theorem in partially ordered sets and some application to matrix equations, Proc. Amer. Math. Soc., 132 (2004), 1435-1443.

[108] A. Razani, A contraction theorem in fuzzy metric space, Fixed Point Theory and Applications, 2005:3 (2005), 257-265.

[109] B.E. Rhoades, A comparison of various definitions of contractive mappings, Trans. Amer. Math. Soc., 226 (1977), 257-290.

[110] J. Rodriguez López and S. Romaguera, The Hausdorff fuzzy metric on compact sets, Fuzzy Sets Syst., 147 (2004), 273-283.

[111] S. Romaguera, A Kirk type characterization of completeness for partial metric spaces, Fixed Point Theory Appl., 2010 (2010), Article ID 493298, 6 pages.

[112] C. Rousseau, Point fixe de Banach (in French), Accromath 5, hiverprintemps, 2010 (www.accromath.ca).

[113] A.R. Roy and P.K. Maji, A fuzzy soft set theoretic approach to decision making problems, J. Comput. Appl. Math., 203 (2007), 412-418.

[114] S. Roy and T.K. Samanta, A note on fuzzy soft topological spaces, Ann. Fuzzy Math. Inf., 3 (2012), 305-311.

[115] R. Saadati, A. Razani and H. Adibi, A common fixed point theorem in $\mathcal{L}-$ fuzzy metric spaces, Chaos, Sol. Fract., 33 (2007), 358-363.

[116] B. Samet, Coupled fixed point theorems for a generalized Meir-Keeler contraction in partially ordered metric spaces, Nonlinear Anal., 72 (2010), 4508-4517. 
[117] B. Schweizer and A. Sklar, Satistical metric spaces, Pacific J. Math., 10 (1960), 314-334.

[118] S. Sessa, On a weak commutativity condition of mappings in fixed point consideration, Publ. Inst. Math. Soc., 32 (1982), 149-153.

[119] M. Shabir and M. Naz, On soft topological spaces, Comput. Math. Appl., 61 (2011), 1786-1799.

[120] B. Singh and M.S. Chauhan, Common fixed points of compatible maps in fuzzy metric spaces, Fuzzy Sets Syst., 115 (2000), 471-475.

[121] S.P. Singh, An application of a fixed point theorem to approximation theory, J. Approx. Theory, 25 (1979), 89-90.

[122] A. Smoluk, Invariant approximation, Mathematyka Stoawana, 17 (1981), 17-22.

[123] T. Som and R.N. Mukherjee, Some fixed point theorems for fuzzy mappings, Fuzzy Sets Syst., 33 (1989), 213-219.

[124] P.V. Subrahmanyam, An Application of a fixed point theorem to best approximation, J. Approx. Theory, 20 (1977), 165-172.

[125] E. Tarafdar, An approach to fixed-point theorems on uniform spaces, Trans. Amer. Math. Soc., 191 (1974), 209-225.

[126] P. Vijayaraju, B.E. Rhoades and R. Mohanraj, A fixed point theorem for a pair of maps satisfying a general contractive condition of integral type, Int. J. Math. Math. Sci., 15 (2005), 2359-2364.

[127] T. Wang, Fixed point theorems and fixed point stability for multivalued mappings on metric spaces, J. Nanjing Univ. Math. Biquart., 6 (1989), $16-23$. 
[128] D. Wardowski, On soft mapping and its fixed points, Fixed Point Theory Appl., 2013:182 (2013), 11 pages.

[129] D. Wardowski, Fixed points of new type of contractive mappings in complete metric spaces, Fixed Point Theory Appl., 2012 (2012), doi: 10.1186/1687-1812-2012-94.

[130] Z. Xiao, K. Gong and S. Xia, Y. Zou, Exclusive disjunctive soft sets, Comput. Math. Appl., 59 (2009), 2128-2137.

[131] Z. Xiao, K. Gong and Y. Zou, A combined forecasting approach based on fuzzy soft sets, J. Comput. Appl. Math., 228 (2009), 326-333.

[132] W. Xu, J. Ma, S. Wang and G. Hao, Vague soft sets and their properties, Comput. Math. Appl., 59 (2010), 787-794.

[133] C.F. Yang, A note on soft set theory, Comput. Math. Appl. 56 (2008), 1899-1900.

[134] X. Yang, T.Y. Lin, J. Yang, Y. Li and D. Yu, Combination of intervalvalued fuzzy set and soft set. Comput. Math. Appl., 58 (2009), 521-527.

[135] M.I. Yazar, C. Gunduz (Aras) and S. Bayramov, Fixed point theorems of soft contractive mappings, arXiv -arxiv.org/pdf/1308.4657 (2013).

[136] L.A. Zadeh, Fuzzy sets, Inf. Control, 8 (1965), 338-353.

[137] S.S. Zhang and Q. Luo, Set-valued Caristi fixed point theorem and Ekeland's variational principle, Appl. Math. Mech. 10 (1989) 111-113 (in Chinese), English translation: Appl. Math. Mech. (English Ed.), 10 (1989), 119-121.

[138] P. Zhu and Q. Wen, Probabilistic soft sets. In: IEEE Conference on Granular Computing, GrC 2010, San Jose, USA, August 14-16, 2010, IEEE, in press (2010). 
[139] P. Zhu and Q. Wen, Operations on soft sets revisited, J. Appl. Math., 2013 (2013), Article ID 105752, 7 pages.

[140] T. Žikić, On fixed point theorems of Gregori and Sapena, Fuzzy Sets Syst., 144 (2004), 421-429.

[141] Y. Zou and Z. Xiao, Data analysis approaches of soft sets under incomplete information, Knowl.-Based Syst. 21 (2008), 941-945. 